University of Tennessee Health Science Center UTHSC Digital Commons

\title{
A Computer Simulation Model of The Human Head-Neck Musculoskeletal System
}

Jun Yan

University of Tennessee Health Science Center

Follow this and additional works at: https://dc.uthsc.edu/dissertations

Part of the Equipment and Supplies Commons, and the Investigative Techniques Commons

\section{Recommended Citation}

Yan, Jun , "A Computer Simulation Model of The Human Head-Neck Musculoskeletal System" (2006). Theses and Dissertations (ETD). Paper 311. http://dx.doi.org/10.21007/etd.cghs.2006.0363. 


\title{
A Computer Simulation Model of The Human Head-Neck Musculoskeletal System
}

\begin{abstract}
Objective. The objective of this research was to develop a muscle-driven biomechanical model of the human head-neck system that could be used to simulate neck movements under muscle control. This model can further be modified to enable input from an external stimulus, such as EMG data.

Summary of background data. Utilizing computer aided design (CAD) and dynamic simulation software programs, the Joint Implant Biomechanics Laboratory at The University of Tennessee Health Science Center developed a virtual model of the human cervical spine to simulate the in vitro biomechanical experiments. This in vitro model did not include any muscle components and was unable to simulate any active muscle contribution to head-neck movement. However, the model served well as a platform from which to develop a dynamic musculoskeletal head-neck model that could include muscle involvement.
\end{abstract}

Methods. The development of the current head-neck model was based on a previous in vitro model of the sub-axial cervical spine that was developed within the rigid body dynamic simulation program, Visual Nastran 4D. Interconnecting joints, including intervertebral discs, facet joints, ligaments, and the C0-C1-C2 complex, were defined. The primary neck muscles for axial rotation, lateral bending, extension, and flexion movements were defined, respectively. For each specific movement, the model was driven by muscle length control using three different muscle sets: (1) all the inclusive primary muscles ("All muscles" mode), (2) only the primary muscles during a concentric contraction ("Concentric contraction muscles only" mode), and (3) only the primary muscles during an eccentric contraction ("Eccentric contraction muscles only" mode). The simulation results obtained from these three modes were compared to the in vivopublished data.

Results. Simulation results from the muscle model for axial rotation and flexion were comparable to the in vivo data in each of the three muscle mode sets. For extension and lateral bending movement, only the results from the "All muscles" mode matched the in vivo data. There were no significant translations that occurred in the upper cervical spine region, which was in agreement with the published literature.

Concluding discussion. A computational model of the human head-neck musculoskeletal system was developed that simulated the dynamic motion response under physiologic head movements. The motiondriven model provided excellent replication of in vivo vertebral kinematics. A similar response occurred for the muscle-driven model when the groups on both sides were activated. Although there was no significant involvement of the extensor muscles during flexion, the forward flexor muscles played an important role during extensional head movement. In the future, the model can be used to explore muscle control strategies within the "Virtual Muscle" program to simulate EMG muscle force activation conditions.

\section{Document Type}

Thesis

\section{Degree Name}

Master of Science (MS)

\section{Program}

Biomedical Engineering 


\section{Research Advisor}

Denis J. DiAngelo, Ph.D.

\section{Keywords}

musculoskeletal model, cervical spine, simulation, neck muscles, biomechanics, virtual muscle

\section{Subject Categories}

Analytical, Diagnostic and Therapeutic Techniques and Equipment | Equipment and Supplies | Investigative Techniques | Medicine and Health Sciences 


\title{
A COMPUTER SIMULATION MODEL OF THE HUMAN HEAD-NECK MUSCULOSKELETAL SYSTEM
}

\author{
A Thesis \\ Presented for \\ The Graduate Studies Council \\ The University of Tennessee \\ Health Science Center \\ In Partial Fulfillment \\ Of the Requirements for the Degree \\ Master of Science \\ In the Joint Graduate Program in Biomedical Engineering \\ From The University of Tennessee \\ and \\ The University of Memphis
}

By

Jun Yan

December, 2006 


\section{DEDICATION}

This thesis is dedicated to my parents

Mr. Jianchu Yan and Mrs. Xiaodong Xu

for their love and support. 


\section{ACKNOWLEDGMENTS}

I would like to thank my advisor, Dr. Denis J. DiAngelo, for the opportunity to learn from his vast knowledge and work in his laboratory. I thank my committee members, Dr. Michael Yen, and Dr. Richard Kasser for helping me with my research. I would also like to express my gratitude to the former Ph.D. student Hyung Soo Ahn who assisted me with the initial phase of this project. I am also grateful to the current and former members in JointImplant Biomechanics Laboratory: Brian Kelly, Brian Morrow, Laura Gilmour, John Shi, and Saurabh Kumar, John Judd, John Schwab, Henry Bonin, and Charles Sullivan.

I also would like to gratefully acknowledge the care and support of my father and mother, who always encouraged me on the way along. 


\section{ASTRACT}

Objective. The objective of this research was to develop a muscle-driven biomechanical model of the human head-neck system that could be used to simulate neck movements under muscle control. This model can further be modified to enable input from an external stimulus, such as EMG data.

Summary of background data. Utilizing computer aided design (CAD) and dynamic simulation software programs, the Joint Implant Biomechanics Laboratory at The University of Tennessee Health Science Center developed a virtual model of the human cervical spine to simulate the in vitro biomechanical experiments. This in vitro model did not include any muscle components and was unable to simulate any active muscle contribution to head-neck movement. However, the model served well as a platform from which to develop a dynamic musculoskeletal head-neck model that could include muscle involvement.

Methods. The development of the current head-neck model was based on a previous in vitro model of the sub-axial cervical spine that was developed within the rigid body dynamic simulation program, Visual Nastran 4D. Interconnecting joints, including intervertebral discs, facet joints, ligaments, and the C0-C1-C2 complex, were defined. The primary neck muscles for axial rotation, lateral bending, extension, and flexion movements were defined, respectively. For each specific movement, the model was driven by muscle length control using three different muscle sets: (1) all the inclusive primary muscles (“All muscles” mode), (2) only the primary muscles during a concentric contraction (“Concentric contraction muscles only” mode), and (3) only the primary muscles during an eccentric contraction ("Eccentric contraction muscles only" 
mode). The simulation results obtained from these three modes were compared to the in vivo published data.

Results. Simulation results from the muscle model for axial rotation and flexion were comparable to the in vivo data in each of the three muscle mode sets. For extension and lateral bending movement, only the results from the "All muscles" mode matched the in vivo data. There were no significant translations that occurred in the upper cervical spine region, which was in agreement with the published literature.

Concluding discussion. A computational model of the human head-neck musculoskeletal system was developed that simulated the dynamic motion response under physiologic head movements. The motion-driven model provided excellent replication of in vivo vertebral kinematics. A similar response occurred for the muscle-driven model when the groups on both sides were activated. Although there was no significant involvement of the extensor muscles during flexion, the forward flexor muscles played an important role during extensional head movement. In the future, the model can be used to explore muscle control strategies within the “Virtual Muscle” program to simulate EMG muscle force activation conditions. 


\section{TABLE OF CONTENTS}

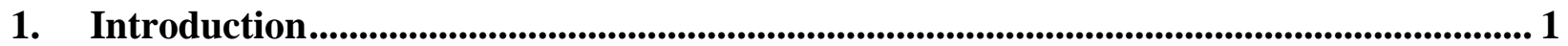

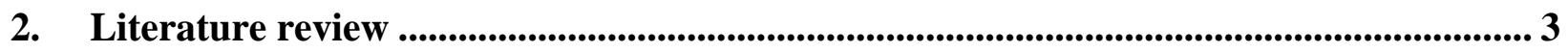

2.1 Functional anatomy of human cervical spine ........................................................ 3

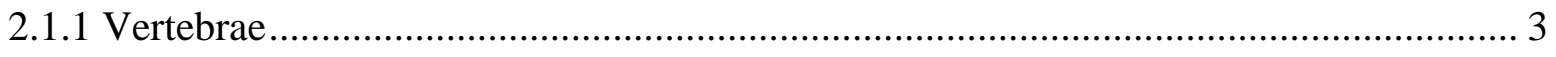

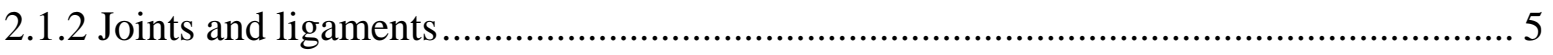

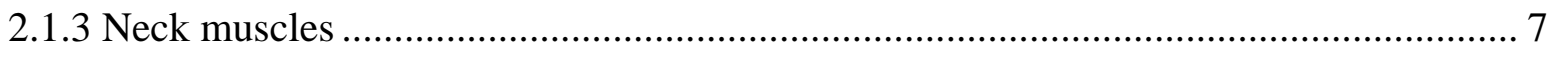

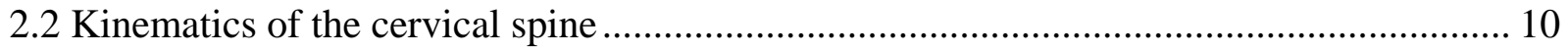

2.3 Current computer modeling techniques of the human head-neck complex...................... 11

2.3.1 Previous simulation work in our biomechanics laboratory................................... 12

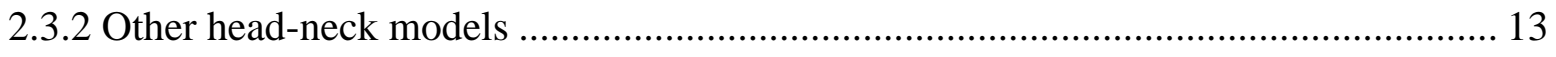

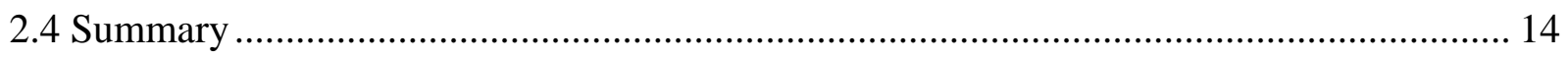

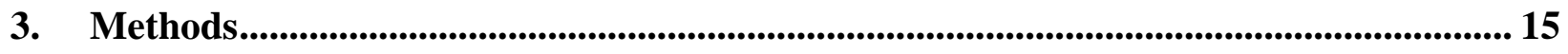

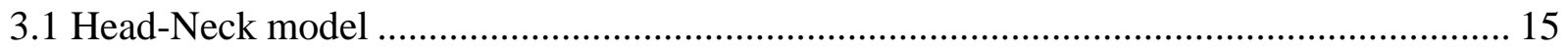

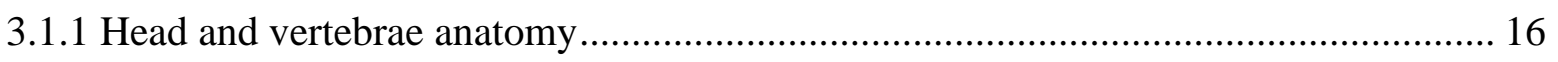

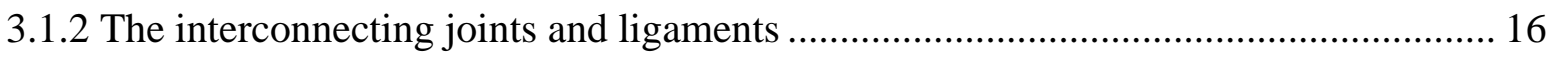

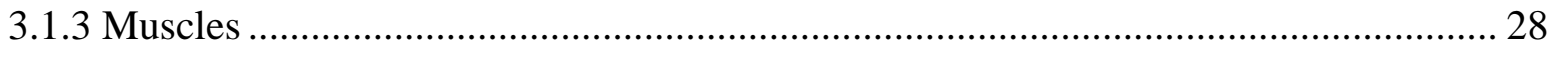

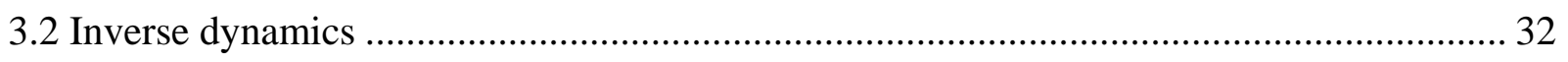

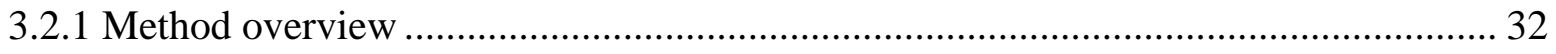

3.2.2 Model driven by motion response................................................................... 34 
3.2.3 Musculoskeletal head-neck system driven under muscle control............................ 36

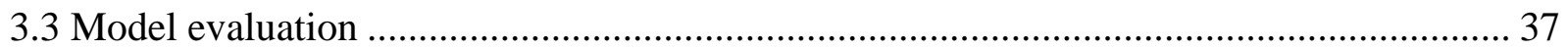

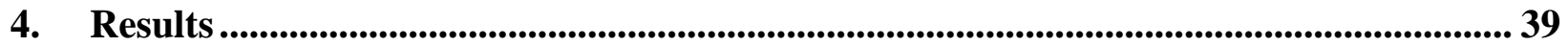

4.1 Simulation of musculoskeletal head-neck complex................................................. 39

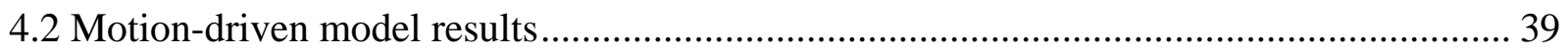

4.2.1 Evaluation of motion-driven model ............................................................... 41

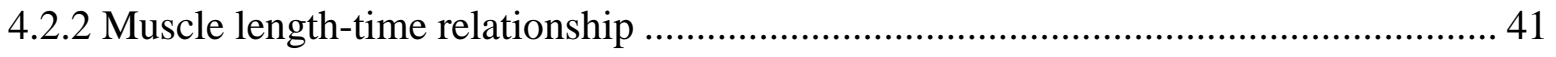

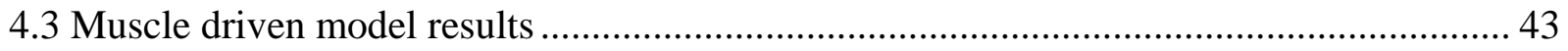

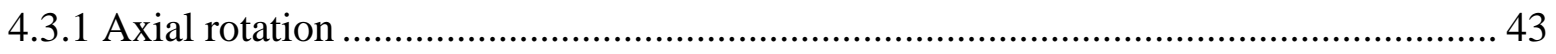

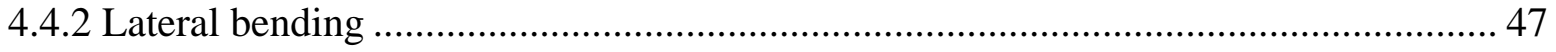

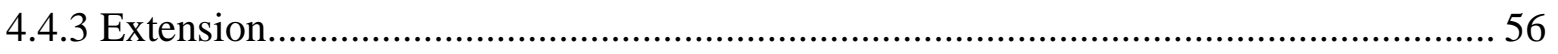

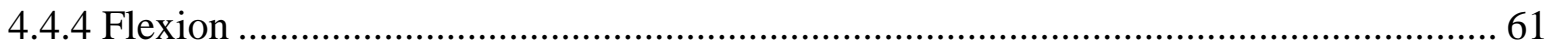

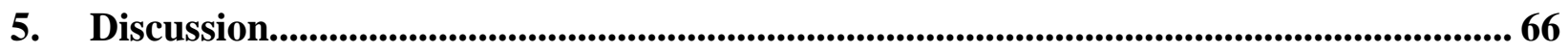

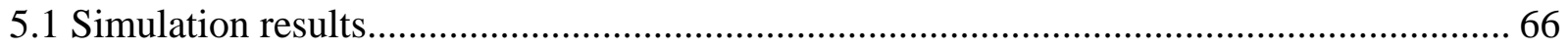

5.2 Comparisons with Ahn model and Vasavada model ................................................ 67

5.3 Muscle selection for axial rotation and lateral bending ............................................. 68

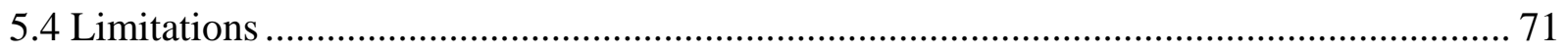

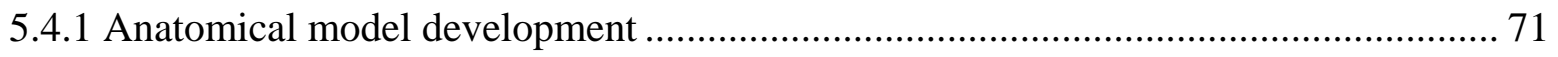

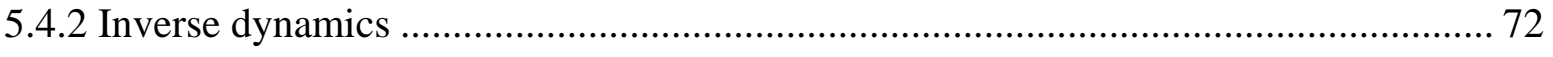

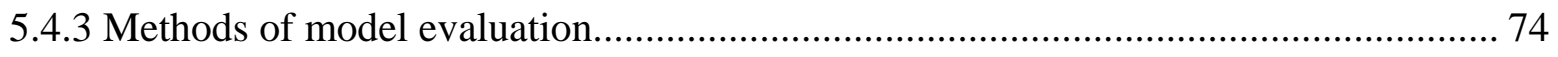


6. Conclusion and future work .................................................................................................. 75

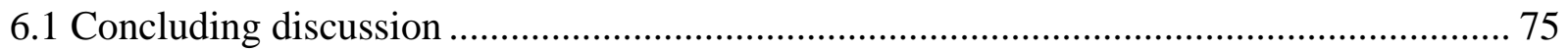

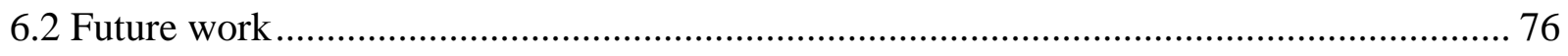

6.2.1 The application of "Virtual Muscle” program .............................................................. 76

6.2.2 Recommendation for improving the anatomical model........................................... 79

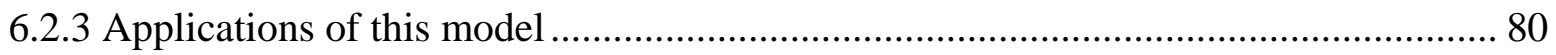

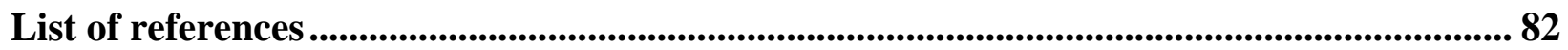

Appendices....................................................................................................................................................... 87

Appendix A Detailed muscle model ..................................................................................... 88

Appendix B Motion segment unit rotations............................................................................. 97

Vita 


\section{LIST OF TABLES}

Table 2-1: Vertebral muscle types according to function and their corresponding

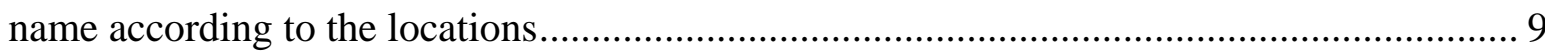

Table 2-2: Representative ranges of motion for in vivo rotation of the cervical spine................ 11

Table 3-1: Inertial and geometric data of vertebral bodies (C1-T1) ...................................... 19

Table 3-2: Coordinate data for C0-C1 motion segment unit moment-rotation curves................ 24

Table 3-3: Coordinate data for C1-C2 motion segment unit moment-rotation curves................ 24

Table 3-4: Coordinate data for the C0-C1 and C1-C2 motion segment units the load-

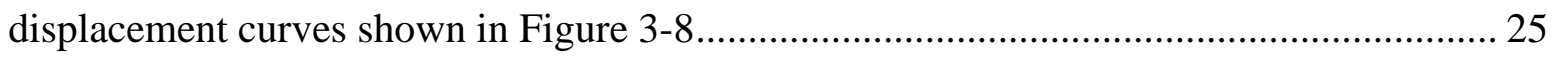

Table 3-5: Neck muscle attachment sites.................................................................... 29

Table 3-6: Primary muscles involved in axial rotation and lateral bending ............................. 30

Table 3-7: Primary muscles involved in extension and flexion............................................ 31

Table 3-8: Comparison of motion-driven model and muscles-driven model ............................ 33

Table 3-9: Muscle behaviors in the three study modes...................................................... 38

Table 4-1: Muscle associated with each muscle activation mode in right-side axial

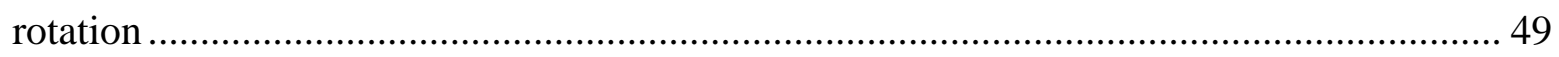

Table 4-2: Muscles associated with each muscle activation mode in left-side lateral

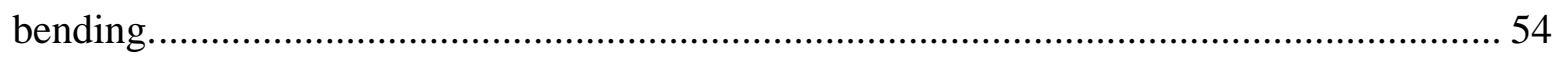

Table 5-1: Comparisons between the current head-neck model, Ahn’s model and Vasavada’s model 


\section{LIST OF FIGURES}

Figure 2-1: Vertebrae of the upper cervical spine. ............................................................. 4

Figure 2-2: Typical vertebral body of the lower cervical spine ........................................ 6

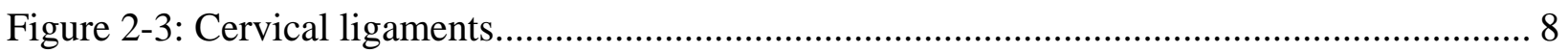

Figure 3-1: Development of the Head-Neck model from the (C2-T1) in vitro model ................ 17

Figure 3-2: Bottom and oblique views of the skull with occipital condyles (C0) ..................... 17

Figure 3-3: Anatomical model of C1 and C2 vertebrae..................................................... 18

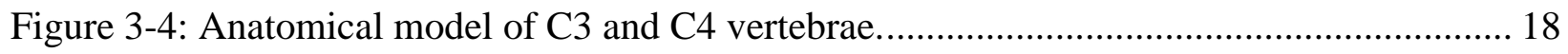

Figure 3-5: The three-dimensional coordinate system for the C0-C1-C2 complex

used to describe rotatory motions .......................................................................... 21

Figure 3-6: Moment-rotation curves for the C0-C1 joint ..................................................... 23

Figure 3-7: Moment-rotation curves for the C1-C2 joint .................................................... 23

Figure 3-8: Load-displacement curves for the C0-C1 and C1-C2 joints .................................. 25

Figure 3-9: The ligaments modeled in the upper cervical spine region.................................. 26

Figure 3-10: The stiffness versus displacement of a ligament............................................. 27

Figure 3-11: The stiffness-displacement curves plot based on Figure 3-10 for the

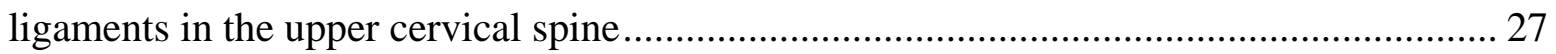

Figure 3-12: Flow chart of the inverse dynamic method................................................... 33

Figure 3-13: The three dimensional coordinate system for the motion driven joints .................. 34

Figure 4-1: Musculoskeletal head-neck simulation model ................................................. 40

Figure 4-2: Comparisons of the segment unit rotations between the simulation

model driven under motion control and in vivo data for different movements. 
Figure 4-3: The rotation versus time curves for each segment level under the

“All muscles” mode in axial rotation.

Figure 4-4: Musculoskeletal head-neck model under “All muscles” mode during right-side axial rotation. 45

Figure 4-5: Musculoskeletal head-neck model under “Concentric contraction muscles only” mode during right-side axial rotation 46

Figure 4-6: Musculoskeletal head-neck model under “Passive elongation muscles only” mode during right-side axial rotation. 48

Figure 4-7: Comparisons of the distribution of relative motion segment unit rotations during axial rotation for the "All muscles” mode, "Concentric contraction muscles only" mode, "Passive elongation muscles only" mode, and in vivo data. 50

Figure 4-8: Musculoskeletal head-neck model under “All muscles” mode during left-side lateral bending. (A) Frontal view. (B) Rear view. 51

Figure 4-9: Musculoskeletal head-neck model under “Concentric contraction muscles only” mode during left-side lateral bending 52

Figure 4-10: Musculoskeletal head-neck model under "Eccentric contraction muscles only” mode during left-side lateral bending 53

Figure 4-11: Comparisons of the distribution of relative motion segment unit rotations during lateral bending for the "All muscles” mode, "Concentric contraction muscles only” mode, "Eccentric contraction muscles only” mode, and in vivo data. 55 
Figure 4-12: Musculoskeletal head-neck model under “All muscles”

mode during extension.

Figure 4-13: Musculoskeletal head-neck model under “Concentric contraction muscles only” mode during extension..... 58

Figure 4-14: Musculoskeletal head-neck model under "Eccentric contraction muscles only” mode during extension. 59

Figure 4-15: Comparisons of the distribution of relative motion segment unit rotations during extension for the "All muscles" mode, "Concentric contraction muscles only” mode, "Eccentric contraction muscles only" mode, and in vivo data. 60

Figure 4-16: Musculoskeletal head-neck model under “All muscles” mode during flexion

Figure 4-17: Musculoskeletal head-neck model under "Concentric contraction muscles only” mode during flexion.

Figure 4-18: Musculoskeletal head-neck model under "Eccentric contraction muscles only” mode during flexion. 64

Figure 4-19: Comparisons of the distribution of relative motion segment unit rotations during flexion for the “All muscles” mode, “Concentric contraction muscles only” mode, "Eccentric contraction muscles only” mode, and in vivo data. 65

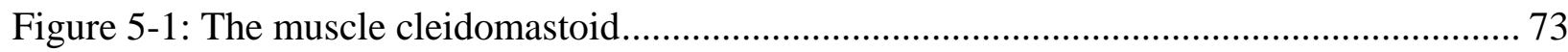

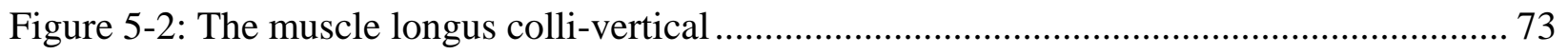

Figure 6-1: Interactions between "Virtual Muscle” and Visual Nastran 4D ............................. 77 
Figure A-1: Muscle longus capitis and colli 89

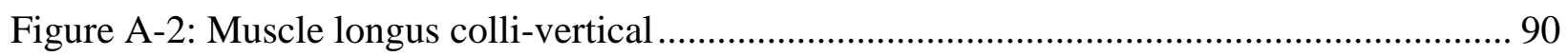

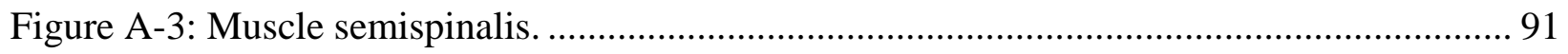

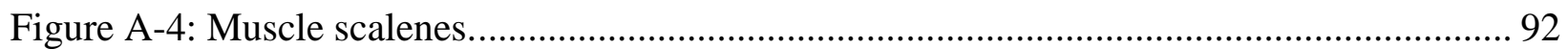

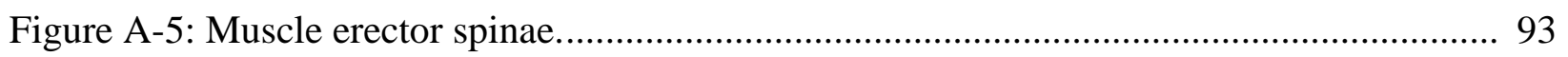

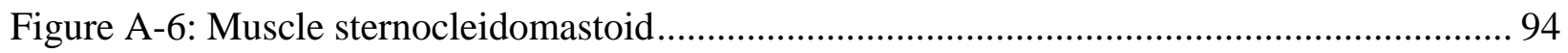

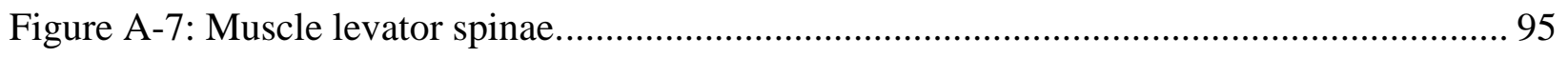

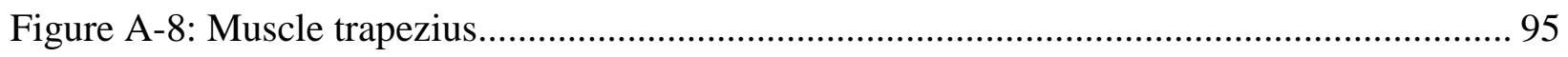

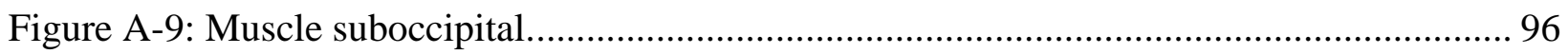

Figure B-1: The rotation versus time curves for each segment level under the "Concentric contraction muscles only" mode in axial rotation ................................... 98

Figure B-2: The rotation versus time curves for each segment level under the "Elongation muscles only" mode in axial rotation ............................................... 99

Figure B-3: The rotation versus time curves for each segment level under the "All muscles only" mode in lateral bending ........................................................ 100

Figure B-4: The rotation versus time curves for each segment level under the "Concentric contraction muscles only" mode in lateral bending. 101

Figure B-5: The rotation versus time curves for each segment level under the "Elongation muscles only" mode in lateral bending. 102

Figure B-6: The rotation versus time curves for each segment level under the "All muscles only" mode in extension. 103

Figure B-7: The rotation versus time curves for each segment level under the "Concentric contraction muscles only" mode in extension 104 
Figure B-8: The rotation versus time curves for each segment level under the "Elongation muscles only" mode in extension 105

Figure B-9: The rotation versus time curves for each segment level under the “All muscles only” mode in flexion. 106

Figure B-10: The rotation versus time curves for each segment level under the "Concentric contraction muscles only" mode in flexion . 107

Figure B-11: The rotation versus time curves for each segment level under the "Elongation muscles only” mode in extension 108 


\section{Introduction}

Since the cervical spine region is a frequent site of injury in the spinal column, research in human neck biomechanics remains one of the most challenging areas in human musculoskeletal system. ${ }^{3,25}$ There are four types of biomechanics models: physical model, in vivo model, in vitro model, and computer model, developed over time to study the cervical spine. ${ }^{25} \mathrm{Ahn}^{3}$ summarized several features of computer models that make them more attractive than other cervical spine research models. Compared to other cervical spine research models, the advantages of computer models are: (1) computer models can provide information that cannot be easily obtained by other models, such as internal stresses or strains, ${ }^{16,26}$ (2) computer models can be used repeatedly for multiple experiments with uniform consistency, which lowers the experimental cost, ${ }^{16}$ and (3) validated computer models can simulate different situations easily and quickly. ${ }^{8,12}$

The Joint Implant Biomechanics Laboratory at The University of Tennessee Health Science Center has developed an in vitro human cervical spine model to investigate the different effects of different surgical techniques (e.g., disc arthroplasty, fusion instrumentation). ${ }^{3}$ The in vitro human cervical spine computer model served as a virtual laboratory simulator for kinematics and kinetics testing. Due to the unavailability of muscle components, the in vitro could not simulate the active muscle motion response. Furthermore, only the cervical vertebrae from C2 to C7, and the interconnecting joints between these two vertebrae, were included in their in vitro model. As a result, their model did not include the attachment site for the muscles, especially for those muscles that attach to the skull, ribcage, or upper cervical vertebrae. 
However, the model developed by $\mathrm{Ahn}^{3}$ served well as the platform from which to further develop a musculoskeletal head-neck model.

In order to fully understand the movement of the head-neck complex, the next step in the Biomechanics Lab is to add muscles to the in vitro model. Once developed, a musculoskeletal head-neck simulation model can provide a better understanding of the complex cervical spine biomechanics and a platform for future model development that integrates muscle excitation functions. The objective of this study was to develop a simulation model of the human headneck complex that included muscles. One aspect of the dynamic muscle model could be used to simulate common head movements, i.e., axial rotation, lateral bending, extension, and flexion under muscle control. Although the muscle excitation functions are not available from the Biomechanics Lab, an EMG-driven model would be developed in the future to estimate muscle forces and neck moment in vivo. ${ }^{20}$

Chapter 2 gives anatomic background information about the human cervical spine and a review of current computational head-neck models. Chapter 3 includes the methodology section that describes soft tissue definition of the upper cervical spine region, neck muscle development, and the method employed to obtain the driving force (muscle length-time relationship) for this head-neck model. Chapter 4 presents the simulation results, including the muscle length-time relationship, and the motion response of each segment. Chapter 5 highlights the findings from this study, and discusses the limitations of this study are also presented. Lastly, the conclusions and future work are provided in Chapter 6. 


\section{Literature review}

This chapter covers three main topics: (1) functional anatomy of human cervical spine, (2) kinematics of human cervical spine, and (3) current status in computer modeling of the human head-neck complex.

\subsection{Functional anatomy of human cervical spine}

A thorough knowledge of human cervical spine functional anatomy is essential to develop a computational model to study spinal kinetics and kinematics. The human cervical spine is the upper part of the spine, which supports the head and protects the spinal cord. Axial rotation, lateral bending, extension, and flexion are the four main motions of the head-neck complex. The basic anatomy of the cervical spine, including the vertebrae, ligaments, facet joints, and muscles, is summarized below.

\subsubsection{Vertebrae}

The human cervical spine consists of seven vertebrae, referred to as C1 through C7. The upper cervical spine consists of occiput (C0), atlas (C1), and axis (C2), and is also called the occipito-atlanto-axial complex (C0-C1-C2). ${ }^{39}$ At the base of the skull, the occiput (C0)

articulates on the atlas (C1) through the convex occiput condyles (OC) (Figure 2-1). ${ }^{11}$ The atlas 

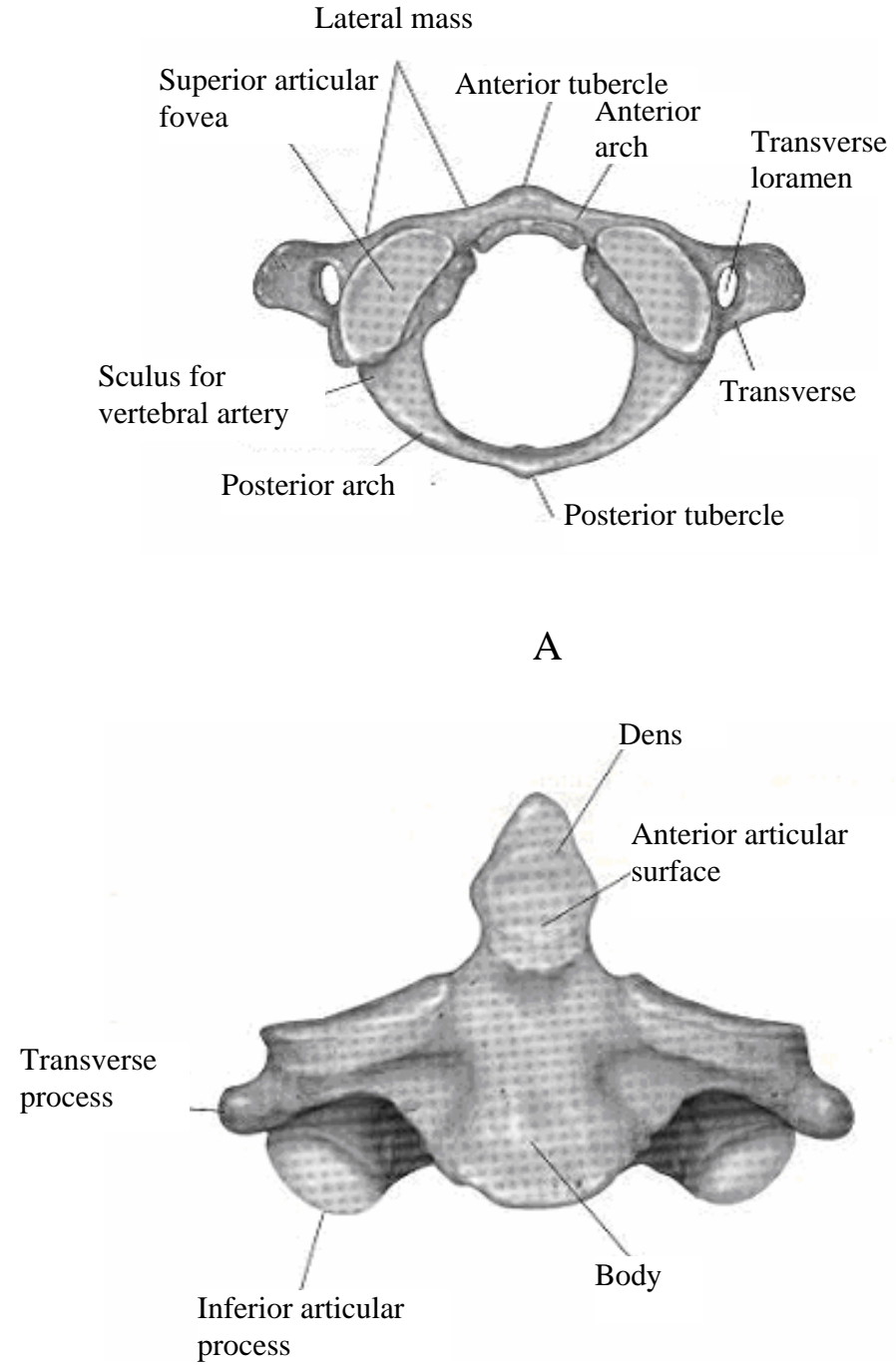

B

Figure 2-1: Vertebrae of the upper cervical spine. (A) Atlas (C1). (B) Axis (C2). (Adapted from Clark et al. and used with permission. Clark CR, ed. The Cervical Spine: The cervical spine research society. 3rd ed. Philadelphia: Lippincott- Raven Publishers, 1998.) 
(C1) is a ring-like structure with anterior and posterior arches where the articular facets and transverse processes are located. However, it lacks a vertebral body and a spinous process. The axis (C2) is characterized by an odontoid process or dens where aoex is rostral from the C2 body to articulate with the posterior aspect of the anterior arch of C1. The C0-C1-C2 complex is the most complicated joint of the skeleton, both anatomically and kinematically. ${ }^{11,41}$ The atlantooccipital (C0-C1) joint lies between the atlas and the occipital condyles of the skull. Between the atlas and the axis, three atlanto-axial (C1-C2) joints occur. Two lateral atlanto-axial joints intervene between the associated facets of the atlas and axis located immediately adjacent to the lateral masses of each vertebra. A median atlantoaxial joint is also present between the dens of the axis and the anterior arch of the atlas. The lower cervical spine is distinct from the upper cervical spine and consists of similar vertebrae from C3 to C7. Each vertebral body in this region has vertebral arch and three processes (one spinous process and two transverse processes)

for muscular and ligamental attachment and articulation (Figure 2-2). ${ }^{11}$ The upper end plate surface is concave from side to side and convex in an anterior-posterior direction, while the lower end plate surface is convex from side to side and concave in an anterior-posterior direction.

\subsubsection{Joints and ligaments}

The intervertebral disc joints, facet joints, and unconvertebral joints consist of joints linking the two adjacent vertebrae in the lower cervical spine region. The intervertebral disc is a fibrocartilaginous joint and allows for six degrees of freedom between two intervertebral bodies. However, there are no discs between axis (C2), atlas (C1), and occiput (C0). The uncovertebral joints, situated on either side of the discs, are small synovial joints located on each side of the 


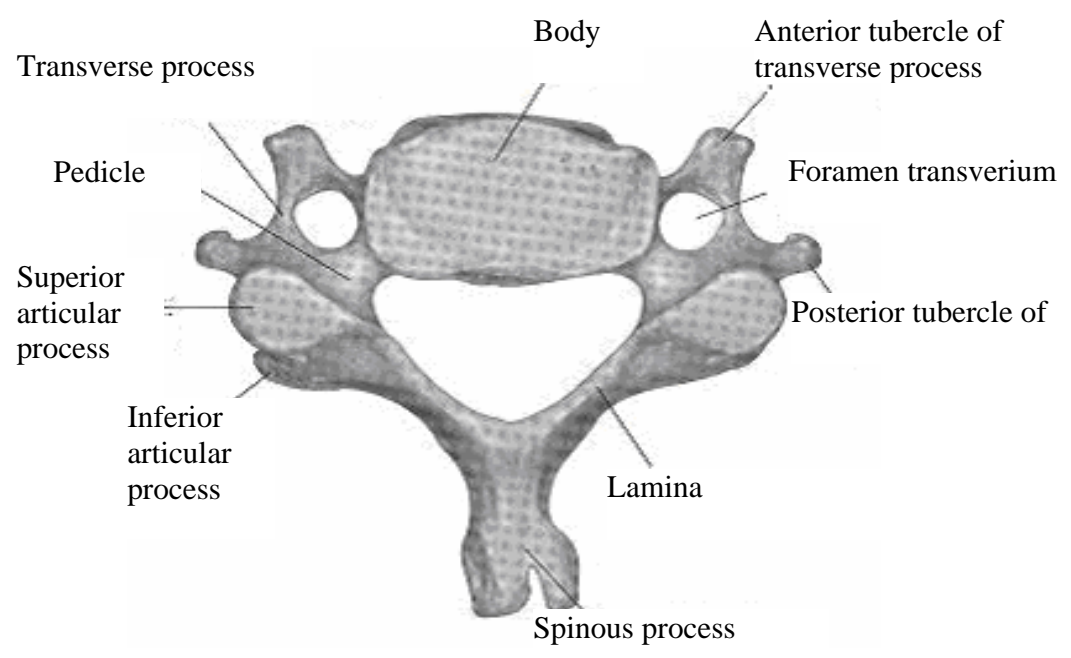

A

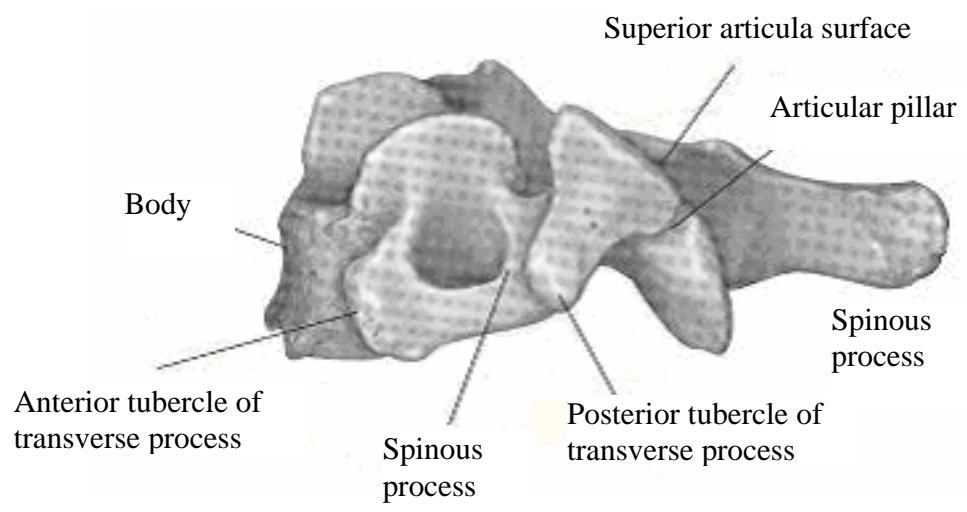

B

Figure 2-2: Typical vertebral body of the lower cervical spine. (A) Cranial view. (B) Lateral view. (Adapted from Clark et al. and used with permission. Clark CR, ed. The Cervical Spine: The cervical spine research society. 3rd ed. Philadelphia: Lippincott- Raven Publishers, 1998.) 
disc. The facet joints are synovial joints formed by the corresponding articular facets of adjacent vertebrae and are enclosed by joint capsules.

Ligaments in the spine protect the spinal cord by allowing spinal motion within the physiologic limits and preventing excess motion. They contain tough flexible collagen rich fibers. The ligaments in the lower cervical spine are shown in Figure 2-3. ${ }^{35}$ The anterior longitudinal ligament (ALL) attaches to the anterior surface of the cervical vertebra body and runs parallel to the length of the spine. The posterior longitudinal ligament (PLL) is located on the posterior aspect of the vertebra body and runs in the direction of the length of the spine. The interspinous ligament and the ligamentum flavum (LF) are located between adjacent spinous processes and adjacent laminae, respectively. The capsular ligament encloses the facet joint. The ligaments in the upper cervical spine are different from those in the lower region (Figure 2-3 B). The anterior occipito-atlantal membrane (AA-OM) and posterior occipito-atlantal membrane (PA-OM) are the continuations of anterior longitudinal ligament (ALL) and ligamentum flavum (LF), respectively. They attach between the skull and the C1 vertebral body. The transverse ligament is a strong horizontal ligament. The apical ligament and the alar ligament hold the dens anteriorly.

\subsubsection{Neck muscles}

There are three main functions of the neck muscles: (1) muscles protect the cervical spine, (2) muscles move the head and neck during the daily living activities, and (3) muscle reflexes stabilize the neck and head in a given posture. Neck muscles are named according to their location, i.e., anterior muscles, lateral muscles, and posterior muscles. Muscles usually appear 


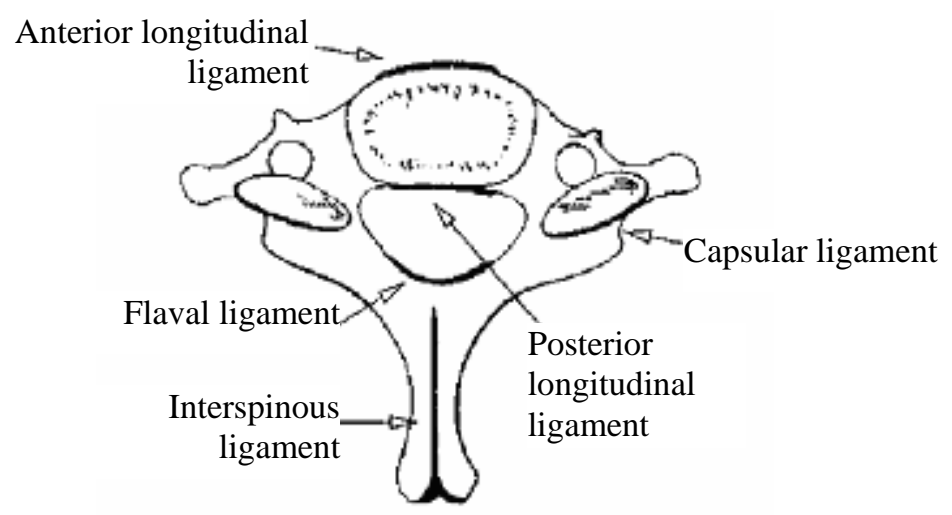

(A)

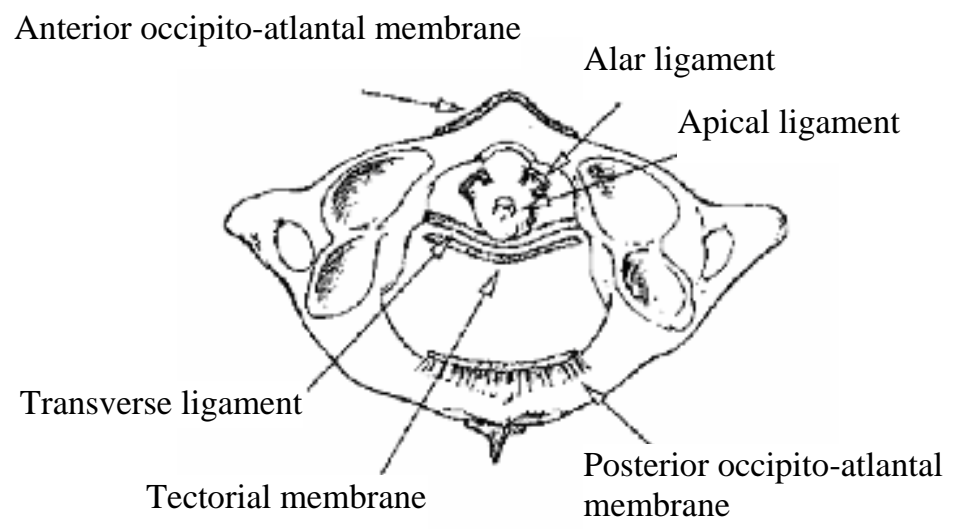

Figure 2-3: Cervical ligaments. (A) Lower cervical ligament. (B) Upper cervical ligament. (Adapted from Sances et al. and used with permission. Sances A, Jr., Myklebust JB, Maiman DJ, et al. The biomechanics of spinal injuries. Crit Rev Biomed Eng 1984;11:1-76.) 
symmetrically throughout the cervical spine. When paired muscles work simultaneously (bilaterally), they either flex or extend the head and neck, or induce lateral bending or axial rotation. The anterior muscles flex the cervical spine, and if they run obliquely and contract independently of the muscles on the opposite side, they will axially rotate the spine as well. Similarly, the posterior muscles in the back extend the spine when they contract, and if they run obliquely and contract independently of their counterpart on the opposite side, they will rotate the spine axially and bend the spine laterally. As lateral muscles contract, they will bend the spine to the same side. Furthermore, the neck muscles are categorized based on their functions as forward flexors (flexion), extensors (extension), rotators (axial rotation), and lateral flexors (lateral bending). According to the general location of the muscles, forward flexor muscles and extensor muscles belong to the anterior muscle group, while the rotator muscles and lateral flexors belong to the lateral muscle group (Table 2-1). ${ }^{6}$

\section{Table 2-1: Vertebral muscle types according to function and their corresponding name according to the locations.}

\begin{tabular}{c|c}
\hline Vertebral Muscles Types (Function) & Vertebral Muscles Types (Location) \\
\hline Forward flexors & Anterior muscles \\
Extensors & Posterior muscles \\
Rotators & Lateral muscles \\
Lateral flexors & Lateral muscles \\
\hline
\end{tabular}

Note: Modified from Bridwell K. Muscles of The Spine.

Website:http://spineuniverse.com/displayarticale.php/article1272.html Access on Feb 272006. 


\subsection{Kinematics of the cervical spine}

In this section, the range of motion of the cervical spine and the coupling motions are introduced. The representative values of the ranges of rotation of the cervical joints, based on various studies, were taken from White and Panjabi (Table 2-2). ${ }^{41}$ Most of the axial rotation occurred in the region between $\mathrm{C} 1$ and $\mathrm{C} 2$, while $\mathrm{C} 0-\mathrm{C} 1$ allowed for most of the flexion/extension and very little axial rotation. About $60 \%$ of the axial rotation of the entire cervical spine and occiput occured in the upper region (C0-C1-C2) and the remainder occurred in the lower region (C2-T1). There was no significant translatory movement at the C0-C1-C2 complex. In the lower cervical spine, most of the flexion/extension occurred in the central region.

There are two main coupled movements in the lower cervical spine that are due to the spatial orientation of the facet joints and the uncovertebral joints. ${ }^{22}$ The first is that flexion is coupled with anterior translation and extension is coupled with posterior translation. The second behavior is the coupled motion between lateral bending and axial rotation. In the upper cervical spine, there is a significant coupling pattern where the axial rotation of $\mathrm{C} 1$ is associated with vertical translation. ${ }^{41}$ 
Table 2-2: Representative ranges of motion for in vivo rotation of the cervical spine.

\begin{tabular}{c|cccccccc}
\hline & \multicolumn{7}{|c}{ Intervertebral joint motions (degree) } \\
Motion & C0-C1 & C1-C2 & C2-C3 & C2-C3 & C3-C4 & C4-C5 & C5-C6 & C7-T1 \\
\hline One side lateral bending & 5 & 5 & 10 & 11 & 11 & 8 & 7 & 4 \\
Combined flexion/extension & 25 & 20 & 10 & 15 & 20 & 20 & 17 & 9 \\
One side axial rotation & 5 & 40 & 3 & 7 & 7 & 7 & 6 & 2 \\
\hline
\end{tabular}

Note: Modified from White AA, Panjabi MM. Clinical Biomechanics of the Spine. 2nd ed. Philadelphia: J.B. Lippincott Company, 1990.

\subsection{Current computer modeling techniques of the human head-neck complex}

Current biomechanical neck models are categorized according to the modeling techniques: finite element models (based on small deflection theory) and rigid multi-body dynamic models. An obvious advantage of the finite element approach is it allows for a more detailed representation of geometry and material properties of the cervical spine. ${ }^{42}$ Several finite element neck models have been published in the past decade. ${ }^{19,37,48}$ In the multi-body dynamic neck models, the head and vertebral bodies are modeled as rigid bodies while the interconnecting tissues, such as intervertebral discs and facet joints, are represented by different joints or springdamper elements. ${ }^{3,14,39}$ Compared to the finite element neck models, multi-body dynamic models are computationally more efficient in the simulation of kinematics and dynamics responses. $^{5,39}$

Over the past few years, multi-body biomechanical neck models $3,14,15,17,39,40$ have been widely used in many fields, such as frontal/lateral acceleration impacts simulation, rear end 
impact simulation, Whiplash Associated Disorders (WAD) research, muscle moment arm influence, and the in vitro experiment protocol simulation.

\subsubsection{Previous simulation work in our biomechanics laboratory}

The Joint Implant Biomechanics Laboratory of the University of Tennessee Health Science Center has made an effort to integrate computer animation and engineering analysis into biomechanical research. Using a general engineering program, Visual Nastran 4D (MSC software, Santa Ana, CA), $\mathrm{Ahn}^{3}$ developed a graphic-oriented multi-body model of the human cervical spine that served as a virtual laboratory simulator for kinematic and kinetic testing. The model included seven rigid bodies from C2 to T1 with detailed definitions of the interconnecting soft tissue structures. The intervertebral discs were modeled as load-based joints with viscoelastic characteristics. The facets joints were defined as joints having compressive stiffness and tensile stiffness. The ligaments were modeled as non-linear spring-damper elements also having viscous behavior. Simulation results from this model were comparable to the in vivo data and the experimental results. One main advantage of the in vitro model developed by Ahn was that it provided a realistic simulation of the in vitro experimental protocol for studying the human cervical spine. As a result, the model could be used to study the kinematics and kinetics of various spine conditions (i.e. fusion, harvested, or intervertebral disc implantation). However, without the muscle components, their model lacked the ability to simulate the in vivo human cervical spine movements. 


\subsubsection{Other head-neck models}

Only a few numerical models of the human cervical spine have been developed that have included the neck muscles. ${ }^{7}$ Brolin $^{7}$ developed a model of the neck that allowed the muscles to curve around the vertebra bodies and included active muscles properties. De Jager ${ }^{14}$ developed a multi-body musculoskeletal head-neck model that consisted of rigid intervertebral bodies, a rigid head, and associated soft tissues. Some representation of active muscle involvement was incorporated into their model. On the basis of De Jager's work, ${ }^{14}$ van der Horst ${ }^{39}$ refined the model with new geometrical description of the cervical spine and new locations of the ligaments and muscles. In van de Horst's model, the muscles could follow the curvature of the neck, which resulted in a more realistic muscle force line of action.

Using a graphic-oriented computer model, an improved kinematics and dynamic simulation could be achieved. Vasavada et al. ${ }^{40}$ created a biomechanical head-neck simulation model capable of representing muscle architecture, musculoskeletal geometry, and cervical spine kinematics. Their model analyzed the effect of muscle morphometry and moment arm lengths on the moment-generating capacity of human neck muscles over physiological ranges of motion. Nineteen distinct muscles were included and the muscle attachments sites were based on anatomical description. The kinematics of the cervical spine were calculated from the “representative angles” of the intervertebral motions reported by White and Panjabi. ${ }^{41}$ Moment arms and force-generating capacity of each neck muscle were calculated for a range of head positions. One limitation of their study was that coupled motions were not included. Another limitation was that all joints were modeled as three degrees of freedom rotational joints having 
no translational components. As a result, their model could only be driven under motion control and could not simulate muscle force control strategies.

\subsection{Summary}

A full understanding of the functional anatomy of human cervical spine is essential to successfully model the human head-neck musculoskeletal system. Modeling techniques provide useful tools for the biomechanical study of human cervical spine. With muscle components

included, the head-neck model can be further developed to simulate in vivo human cervical spine movement through the incorporation with muscle control programs. 


\section{Methods}

In this chapter, a three-dimensional mathematical model of the human head-neck musculoskeletal system was developed. This model studied the motion responses of the human cervical spine under the control of muscles. The graphics-oriented multi-body human head-neck model consisted of three major parts: head and vertebrae, interconnecting joints and ligaments, and muscles. These three components are discussed in detail in the first Section (3.1). The second Section (3.2) describes the application of using inverse dynamics. A motion responsedriven model and a muscle-driven model were developed. The muscle changes determined from the motion-driven model served as input for the model driven by muscle control. The muscle driven model was studied under three different muscle sets, which are also discussed in this section. Lastly, the method used to evaluate the model output is discussed in the Section (3.3).

\subsection{Head-Neck model}

The model was developed within a rigid body dynamic simulation program, Visual Nastran 4D. The model developed by $\mathrm{Ahn}^{3}$ served as the basis for this research. The soft tissues of upper cervical spine region were defined and the muscle components of the head-neck complex were incorporated into the in vitro computational model. 


\subsubsection{Head and vertebrae anatomy}

The head-neck musculoskeletal model was developed based on the in vitro cervical spine (C2-T1) model developed by Ahn. ${ }^{3}$ Their anatomical model is shown in Figure 3-1. The skull, C1, clavicle, the scapula, and upper part of the thorax were added to their model. The geometry of the skeletal components, such as skull, cervical vertebrae, and thorax was created from axial computer tomography (CT) images obtained from National Library of Medicine’s Visible Human data set. ${ }^{1}$ Image processing software SliceOmatic 4.2 (Tomovision, Montreal, Canada) and surface modeling software Geomagic Studio 5 (Raindrop Geomagic, Research Triangle Park, NC) were also used to create the surface models before they were imported to Visual Nastran 4D (MSC software, Santa Ana, CA). ${ }^{3}$ Figure 3-2 to Figure 3-4 show the detailed model of skull (Figure 3-2), C0 (Figure 3-2), C1 (Figure 3-3 A), C2 (Figure 3-3 B), and the remaining vertebrae (Figure 3-4). In this model, the scapula and thorax were fixed and used only for muscular attachment, while the mass, the moment of inertia, and the position of the center of mass for the vertebral bodies were obtained from Visual Nastran 4D software and are listed in Table 3-1.

\subsubsection{The interconnecting joints and ligaments}

The interconnecting joints and ligaments were defined relative to the region they belong to: the lower sub-axial spine region (C2-T1) or the upper cervical spine region (C0-C2). 

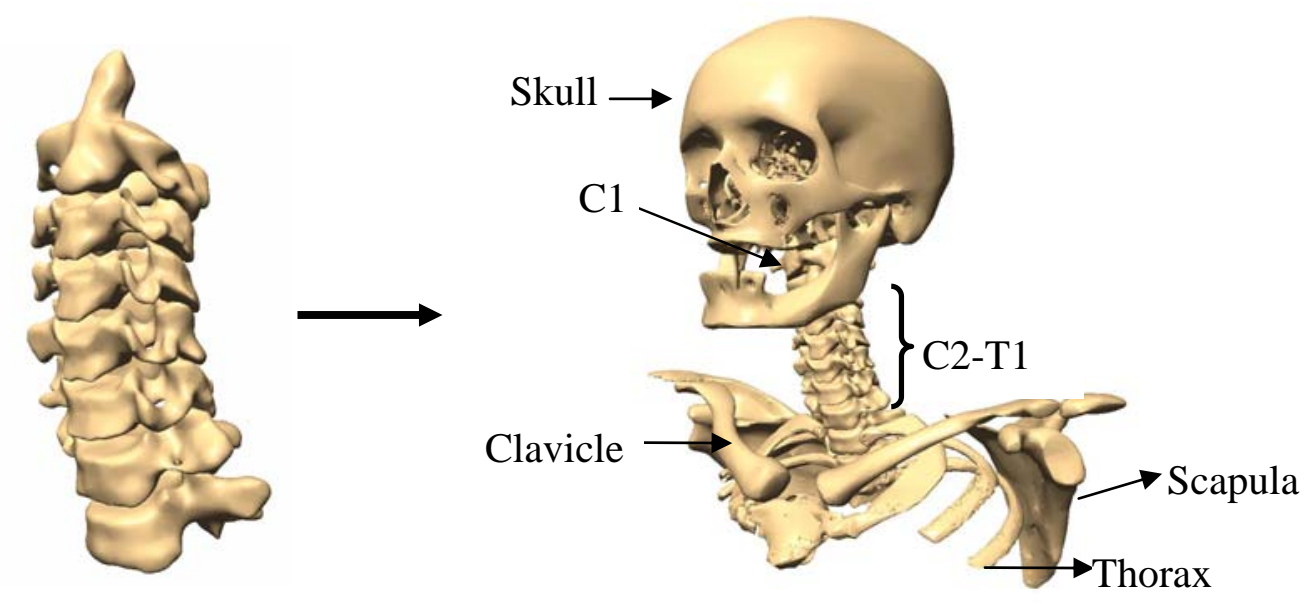

Figure 3-1: Development of the Head-Neck model from the (C2-T1) in vitro model. (A) Original in vitro model (C2-T1). (B) The current head-neck model.
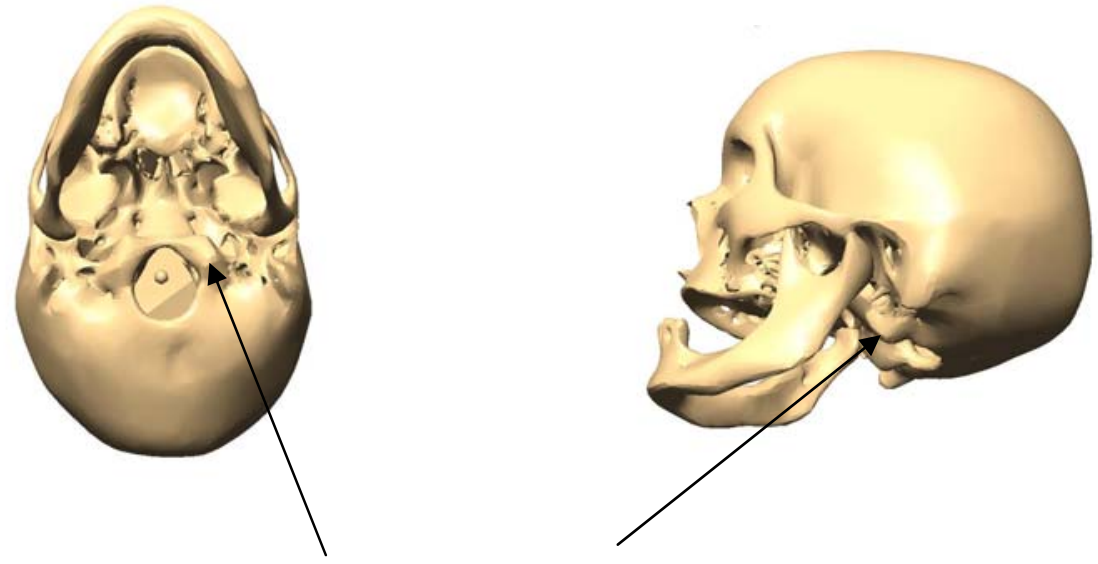

Occipital Condyles (OC)

Figure 3-2: Bottom and oblique views of the skull with occipital condyles (C0). 


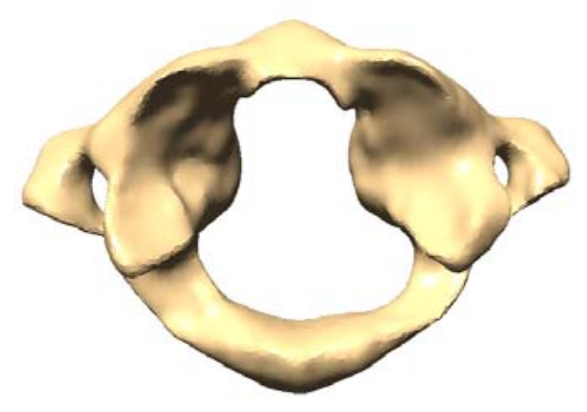

A

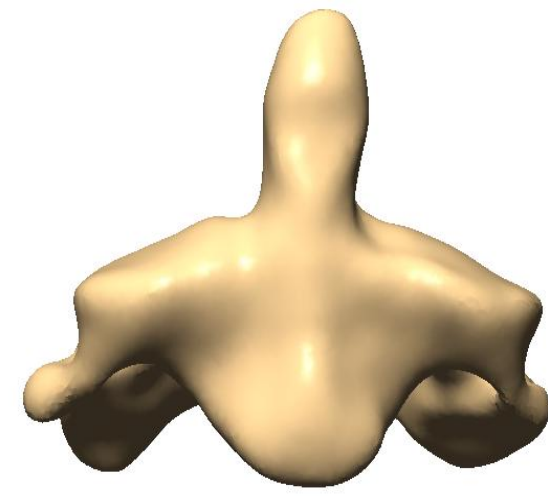

B

Figure 3-3: Anatomical model of C1 and C2 vertebrae. (A) C1. (B) C2.

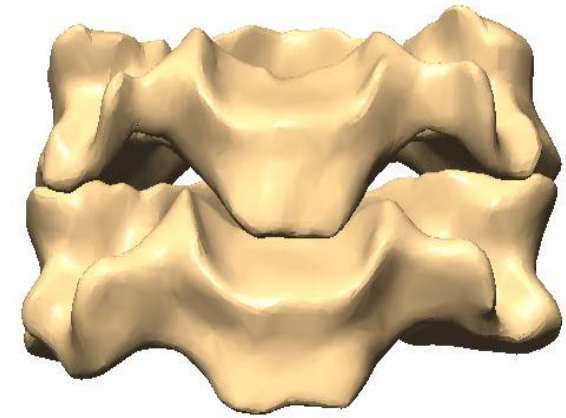

A

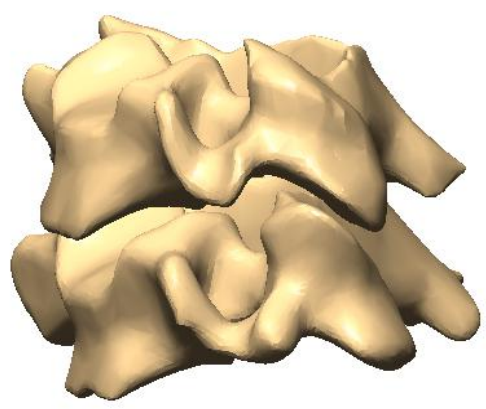

B

Figure 3-4: Anatomical model of C3 and C4 vertebrae. (A) Anterior view. (B) Lateral view. 
Table 3-1: Inertial and geometric data of vertebral bodies (C1-T1).

\begin{tabular}{|c|c|c|c|c|c|}
\hline \multirow{2}{*}{$\begin{array}{c}\text { Vertebral } \\
\text { body }\end{array}$} & \multirow{2}{*}{$\begin{array}{l}\text { Mass } \\
(\mathrm{kg})\end{array}$} & \multicolumn{3}{|c|}{ Moment of inertia $\left(\mathrm{kgmm}^{2}\right)$} & \multirow{2}{*}{$\begin{array}{l}\text { Center of mass position } \\
\text { relative to } \mathrm{T} 1 \text { center of } \\
\text { mass (mm) (X: right, Y: } \\
\text { anterior, } \mathrm{Z} \text { : upward) }\end{array}$} \\
\hline & & $I_{X X}$ & $I_{y y}$ & $I_{z z}$ & \\
\hline C1 & 0.21 & 29.3 & 81.6 & 99.0 & $(2.24,33.81,111.00)$ \\
\hline $\mathrm{C} 2$ & 0.25 & 46.3 & 47.7 & 64.9 & $(3.29,32.61,93.80)$ \\
\hline C3 & 0.26 & 23.7 & 48.9 & 65.3 & $(4.35,32.50,75.29)$ \\
\hline $\mathrm{C} 4$ & 0.27 & 23.8 & 57.7 & 72.2 & $(4.17,28.96,61.18)$ \\
\hline C5 & 0.26 & 24.3 & 61.2 & 73.4 & $(3.19,25.20,46.64)$ \\
\hline C6 & 0.29 & 38.8 & 62.7 & 79.0 & $(1.51,18.40,31.97)$ \\
\hline C7 & 0.41 & 72.1 & 89.2 & 132.8 & $(1.34,8.51,16.40)$ \\
\hline $\mathrm{T} 1$ & 0.27 & 50.4 & 68.7 & 93.9 & $(0.00,0.00,0.00)$ \\
\hline
\end{tabular}




\subsubsection{Definition of joints in lower cervical spine}

The joints connecting any two vertebral bodies in the lower cervical spine region (C2-C7) were described as "triple joint complexes" in the in vitro model developed by Ahn. ${ }^{3}$ The triple joint complexes were comprised of intervertebral disc joints in the anterior region, two facet joints in the posterior region, and ligament structures between two vertebrae. This definition was also adapted in the current head-neck musculoskeletal model.

\subsubsection{Definition of joints in upper cervical spine}

The rigid bodies of the upper cervical spine formed by C0, C1, and C2 were connected by intervertebral joints and represented the compound behaviors of occipital (C0)-atlano (C1)-axial (C2) complex. The joints included ligaments and facet joints. In this study, the stiffness properties of the transverse ligament, alar ligament, the apical ligament, and the facet joints in the upper region were lumped together in Visual Nastran 4D to form generic joints for C0-C1 and C1-C2. Both joints had six degrees of freedom as shown in Figure 3-5. To be consistent with previous ligaments development in the lower cervical spine, ${ }^{3}$ four types of ligaments were modeled: anterior atlanto-occipital membrane (AA-OM), posterior atlanto- occipital membrane (PA-OM), anterior longitudinal ligament (ALL), and ligamentum flavum (LF).

The origin of the coordinate system for $\mathrm{C} 0-\mathrm{C} 1$ joint was set above the occipital condyles (Figure 3-5 A), while the origin of the coordinate system for C1-C2 joint was located at twothirds the height of the dens along the central axis of the dens (Figure 3-5 B). The Xd, Yd, and Zd- axes for both C0-C1 and C1-C2 joints (Figure 3-5) were assigned pointing right laterally, 


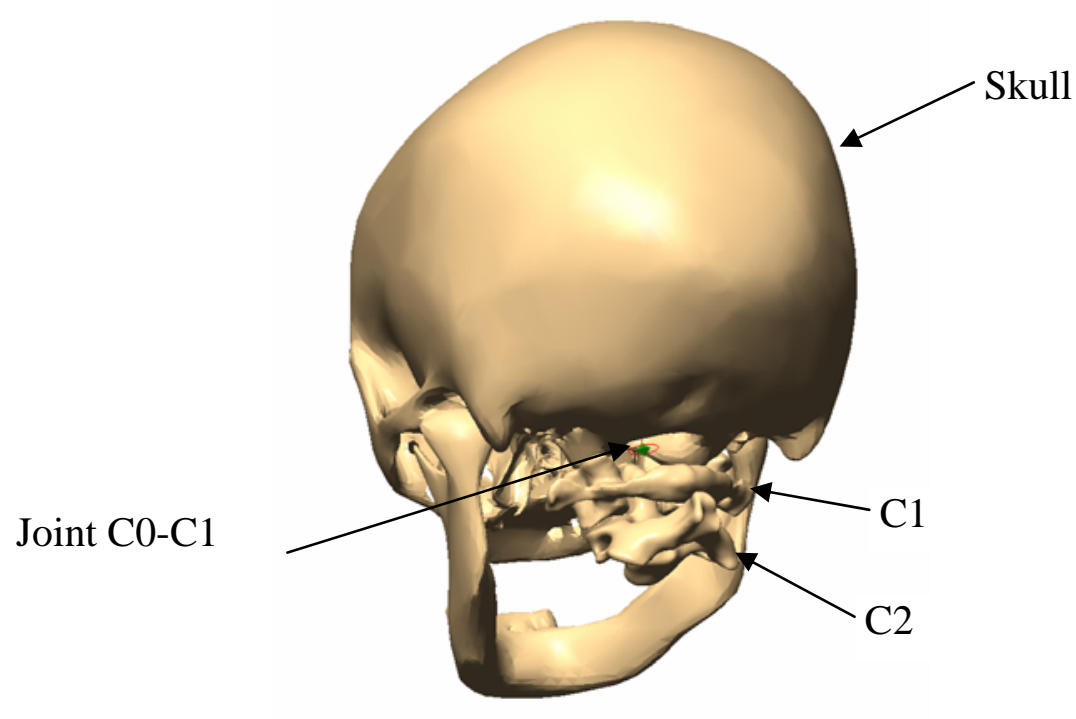

(A)

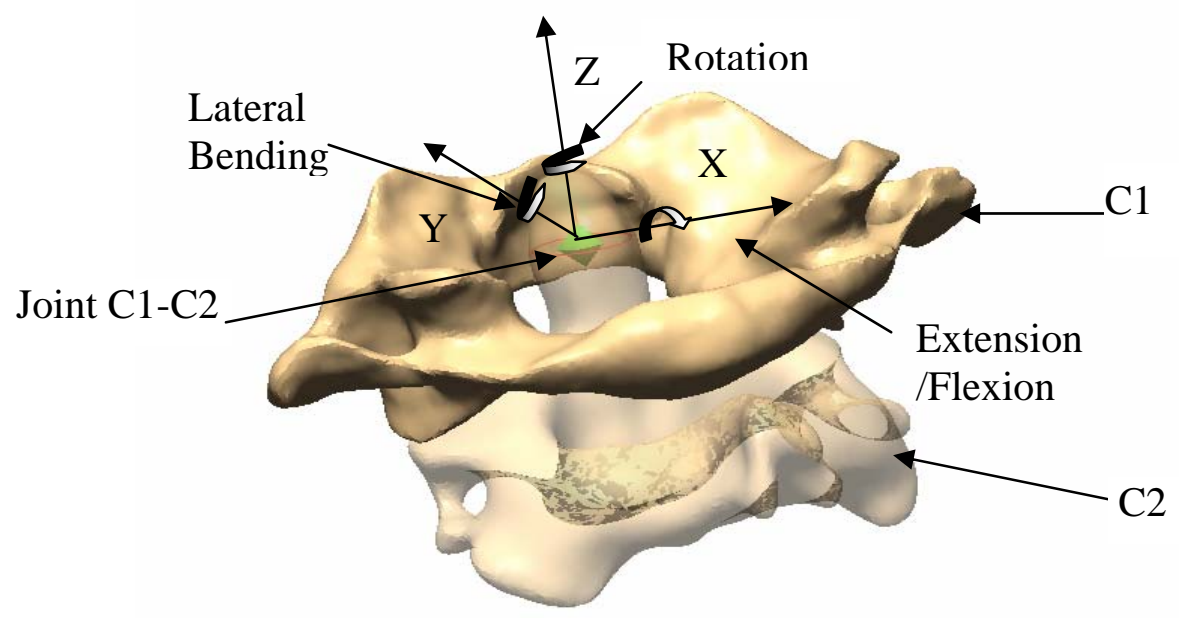

(B)

Figure 3-5: The three-dimensional coordinate system for the C0-C1-C2 complex used to describe rotatory motions. (A) $\mathrm{C} 0-\mathrm{C} 1$ joint. (B) $\mathrm{C} 1-\mathrm{C} 2$ joint. 
anteriorly, and cranially, respectively.

Viscous behavior of the C0-C1, C1-C2 generic joints was defined by constant damping coefficients obtained from De Jager's study. ${ }^{14}$ The damping coefficients varied for different motions. For example, the damping coefficients were $1 \mathrm{Ns} / \mathrm{mm}$ for translations and 26 Nmms/deg for rotation.

In order to model the elastic behavior of the joints, load-displacement curves were assigned for each degree of freedom and each level of C0-C1 and C1-C2 joints. The rotational stiffness of C0-C1 and C1-C2 joints were defined by nonlinear moment- rotation curves. ${ }^{14}$ Figure 3-6 and Figure 3-7 show the moment-rotation curves that represent the in vitro elastic behavior of C0-C1 and C1-C2 joints, respectively. Table 3-2 and Table 3-3 provide the corresponding data.

De Jager ${ }^{14}$ used a relatively large stiffness of $500 \mathrm{~N} / \mathrm{mm}$ to define the translatory stiffness in his model, as no force-translation data were available in the literature. In this study, the translatory stiffness values for the upper cervical spine joints were assumed to be identical to that used for the lower cervical spine intervertebral discs. These stiffness values resulted in minimal translational motion for normal physiologic loads, which agreed with the physical observations for these joints. As described in detail in Ahn's dissertaion, ${ }^{3}$ force-translation curves for Xd- and Yd- directions (shear) were adapted and modified from De Jager, ${ }^{14}$ while the force-translation curve for Zd- direction (tension-compression) were directly adapted from Shea et al.. ${ }^{36}$ Figure 3-8 shows the force-translation curves for C0-C1 joint and C1-C2 joint. The corresponding data are given in Table 3-4. 


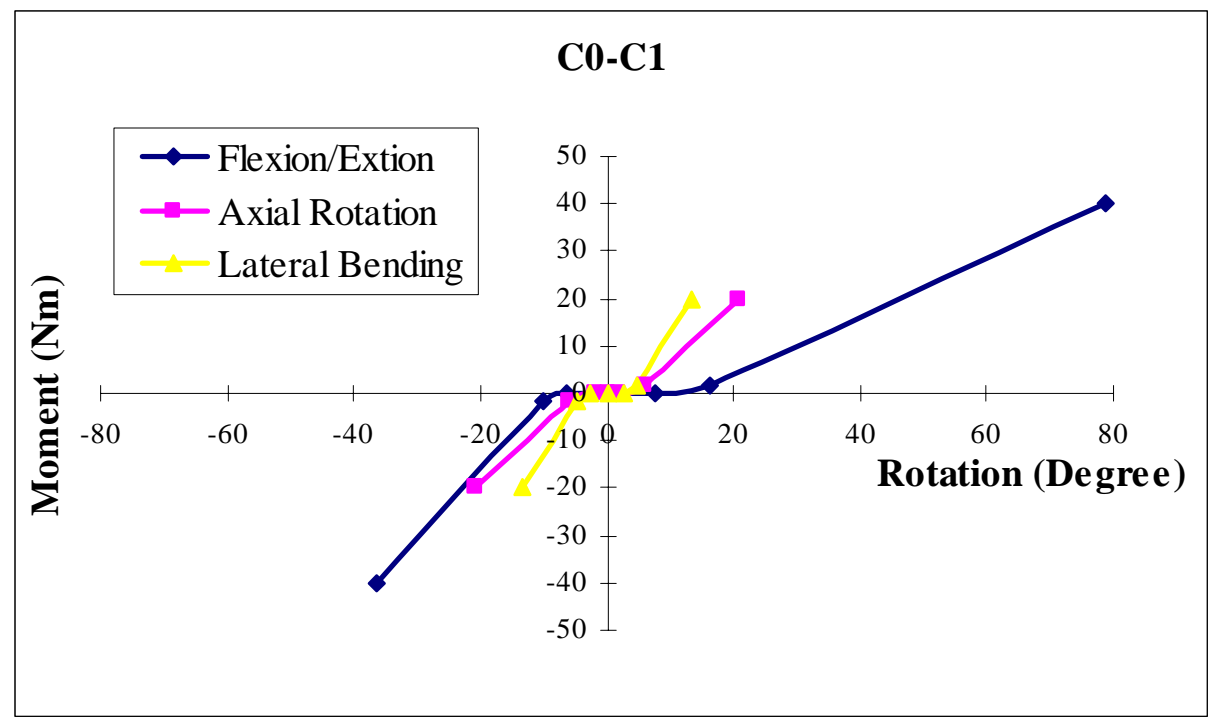

Figure 3-6: Moment-rotation curves for the C0-C1 joint. (Adapted from De Jager MKJ. Mathematical Head-Neck Model for Acceleration Impacts: Eindhoven University of Technology, 1996)

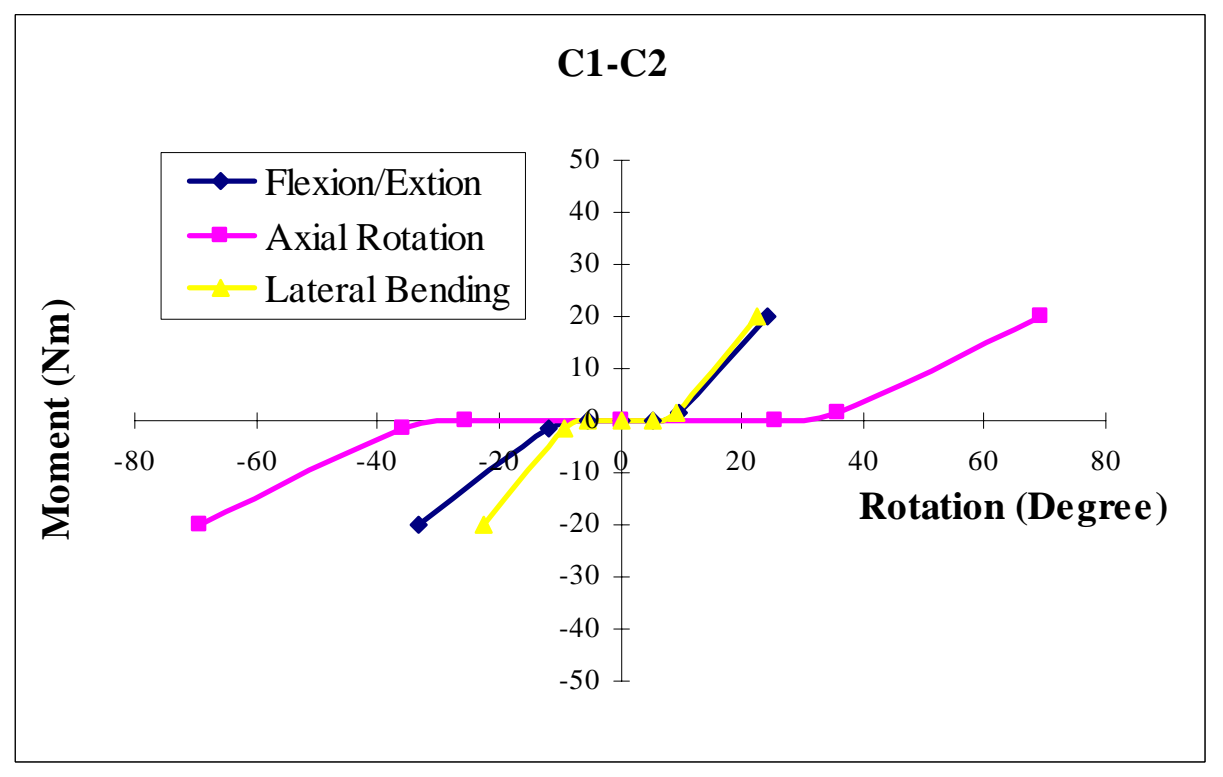

Figure 3-7: Moment-rotation curves for the C1-C2 joint. (Modified from De Jager MKJ. Mathematical Head-Neck Model for Acceleration Impacts: Eindhoven University of Technology, 1996) 
Table 3-2: Coordinate data for $\mathrm{C} 0$-C1 motion segment unit moment-rotation curves.

\begin{tabular}{c|c|c|c|c|c}
\hline \multicolumn{2}{c|}{ C0-C1 } \\
\hline \multicolumn{2}{c|}{ Axial Rotation } & \multicolumn{2}{c}{ Lateral Bending } & \multicolumn{2}{c}{ Flexion /Extension } \\
\hline Deg & $\mathrm{Nm}$ & Deg & Nm & Deg & Nmm \\
\hline-20.8 & -20 & -13.4 & -20. & -36.6 & -40 \\
-6.1 & -1.5 & -4.9 & -1.5 & -10.2 & -1.5 \\
-1.8 & -0.15 & -2.5 & -0.15 & -6.5 & -0.15 \\
1.8 & 0.15 & 2.5 & 0.15 & 7.6 & 0.15 \\
6.1 & 1.5 & 4.9 & 1.5 & 16.3 & 11.5 \\
20.8 & 20 & 13.4 & 20 & 78.7 & 40 \\
\hline
\end{tabular}

Note: Modified from De Jager MKJ. Mathematical Head-Neck Model for Acceleration Impacts: Eindhoven University of Technology, 1996

Table 3-3: Coordinate data for C1-C2 motion segment unit moment-rotation curves.

\begin{tabular}{c|c|c|c|c|c}
\hline \multicolumn{6}{c|}{ C1-C2 } \\
\hline Axial Rotation & \multicolumn{2}{c}{ Lateral Bending } & \multicolumn{2}{c}{ Flexion/ Extension } \\
\hline Deg & Nmm & Deg & Nmm & Deg & Nmm \\
\hline-69.4 & -20 & -22.7 & -20 & -33.1 & -20 \\
-35.8 & -1.5 & -9.3 & -1.5 & -11.7 & -1.5 \\
-25.6 & -0.15 & -5.4 & -0.15 & -5.5 & -0.15 \\
25.6 & 0.15 & 5.4 & 0.15 & 5.3 & 0.15 \\
35.8 & 1. & 9.3 & 1.5 & 9.6 & 1.5 \\
69.4 & 20 & 22.7 & 20 & 24.1 & 20 \\
\hline
\end{tabular}

Note: Modified from De Jager MKJ. Mathematical Head-Neck Model for Acceleration Impacts: Eindhoven University of Technology, 1996 


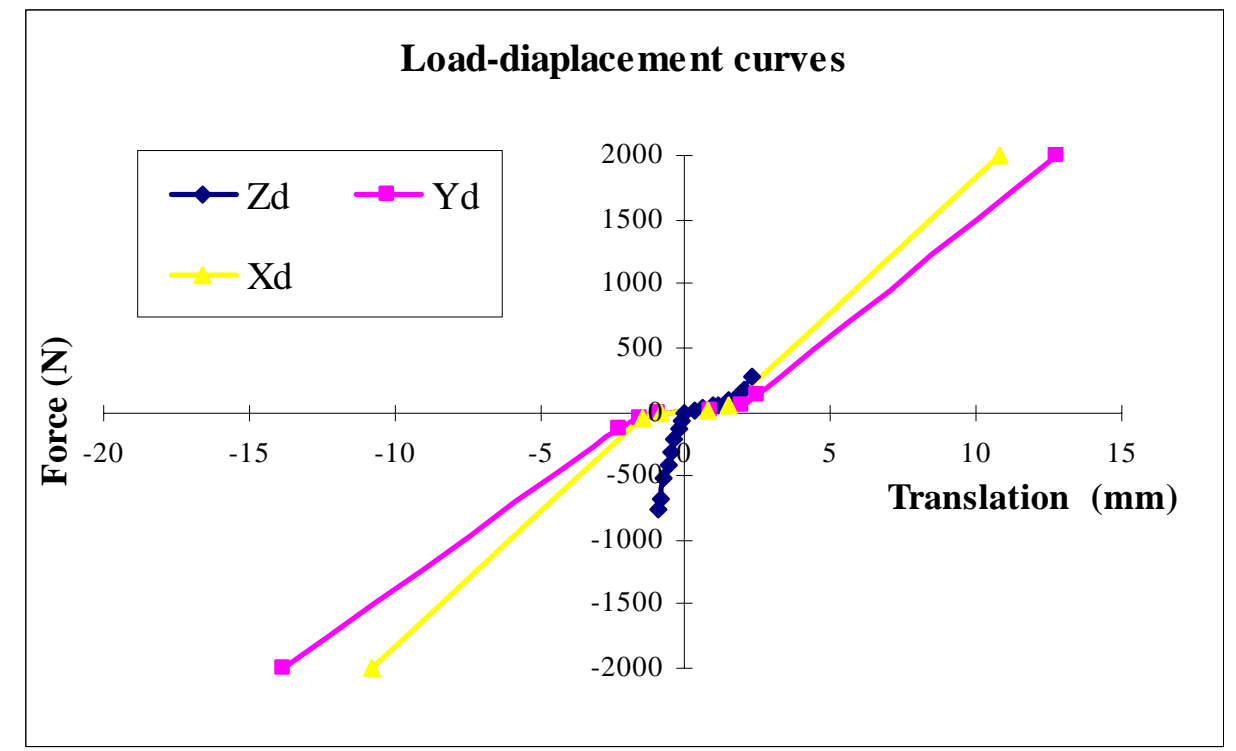

Figure 3-8: Load-displacement curves for the C0-C1 and C1-C2 joints. (Modified from De Jager MKJ. Mathematical Head-Neck Model for Acceleration Impacts: Eindhoven University of Technology, 1996)

Table 3-4: Coordinate data for the CO-C1 and C1-C2 motion segment units the loaddisplacement curves shown in Figure 3-8.

\begin{tabular}{c|cccccccc}
\hline \multicolumn{10}{c}{ Zd } \\
\hline Translation (mm) & -0.936 & -0.863 & -0.721 & -0.608 & -0.475 & -0.377 & -0.23 & -0.132 \\
Force (N) & -757.27 & -689.33 & -524.28 & -415.63 & -308.73 & -207.84 & -133.99 & -75.725 \\
Translation (mm) & 0.328 & 0.613 & 0.902 & 1.162 & 1.461 & 1.765 & 2.054 & 2.309 \\
Force (N) & 19.417 & 38.882 & 42.761 & 58.282 & 83.514 & 120.4 & 182.53 & 275.72 \\
\hline \multicolumn{7}{c}{ Yd } \\
\hline Translation (mm) & -13.8 & -2.3 & -1.6 & -0.9 & 0.9 & 1.9 & 2.5 & 12.8 \\
Force (N) & -2000 & -125 & -50 & -5 & 5 & 50 & 125 & 2000 \\
\hline \multicolumn{7}{c}{ Xd } \\
Translation (mm) & -10.8 & -1.5 & -0.8 & 0.8 & 1.5 & 10.8 & & \\
Force (N) & -2000 & -50 & -5 & 5 & 50 & 2000 & & \\
\hline
\end{tabular}

Note: Modified from De Jager MKJ. Mathematical Head-Neck Model for Acceleration Impacts:

Eindhoven University of Technology, 1996 
The ligaments, anterior atlanto-occipital membrane (AA-OM), posterior atlanto-occipital membrane (PA-OM), anterior longitudinal ligament (ALL), and ligamentum flavum (LF) were modeled as non-linear spring-damper systems that resisted tensile or distractive forces (Figure 3-9), similar to the method used to define ligaments in the lower cervical spine region. ${ }^{3}$ Since AA-OM, PA-OM, and ALL are narrower at the upper level, ${ }^{28}$ they were modeled as two-parallel spring-damper elements compared to the ligaments ALL and PLL in the lower cervical spine, which were modeled as three parallel spring-damper elements. ${ }^{3}$ A three-parallel spring-damper element was used to define LF located between C1 and C2. The origin and insertion of ligaments were located at the margin of the vertebrae. ${ }^{3}$

The viscous behavior for the ligaments was represented by a constant damping coefficient of $0.4 \mathrm{NS} / \mathrm{mm}$ that was adapted from van der Horst ${ }^{39}$ and Yoganandan et al. ${ }^{45}$ The stiffness-displacement curves (Figure 3-10) represent the non-linear elastic behavior of each ligament. The data were derived from published literatures ${ }^{44}$ and plotted in Figure 3-11.

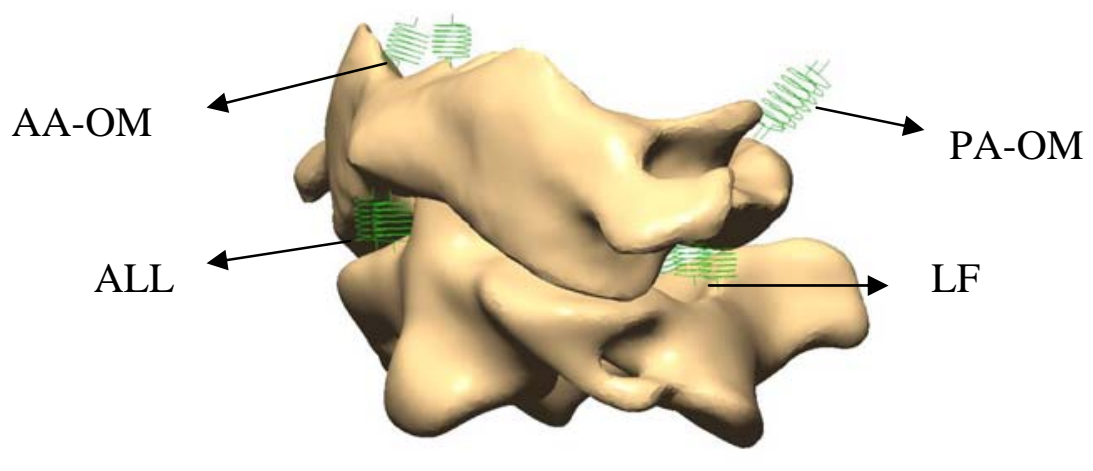

Figure 3-9: The ligaments modeled in the upper cervical spine region. 


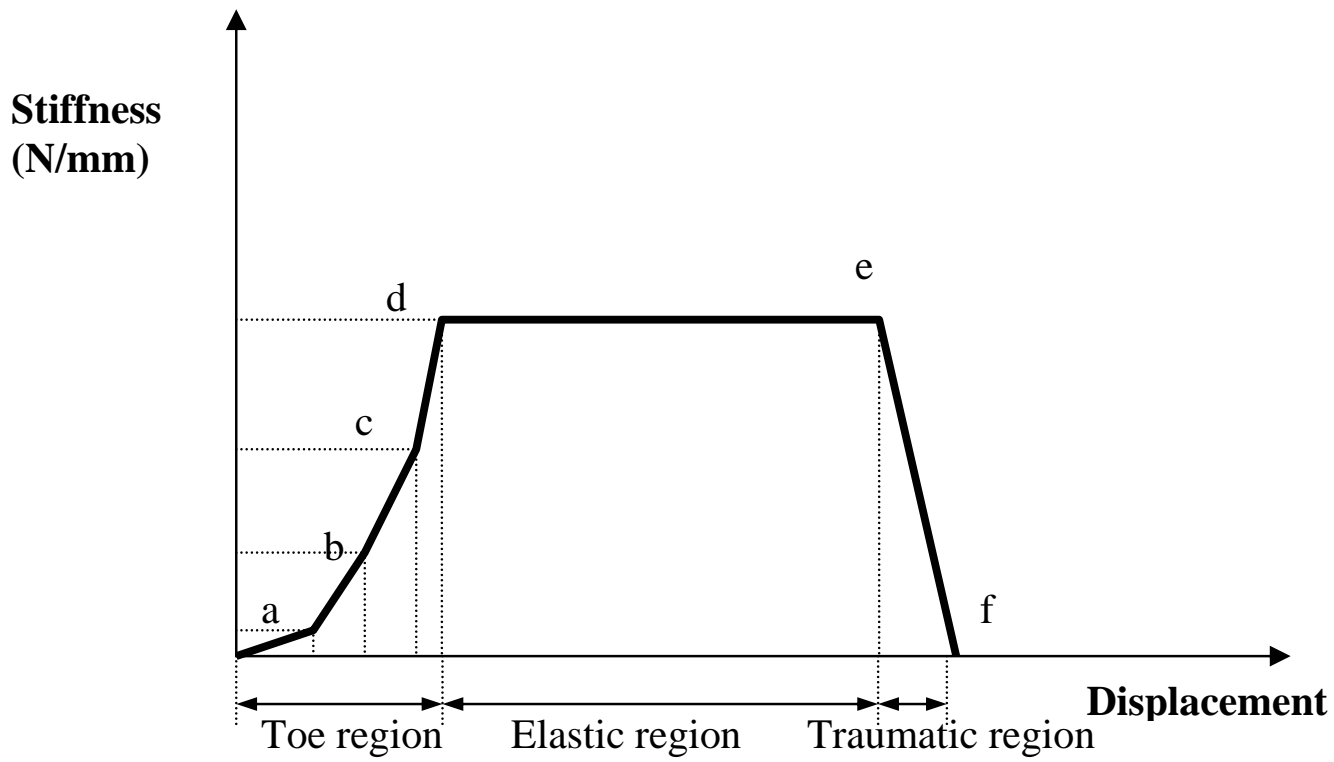

Figure 3-10: The stiffness versus displacement of a ligament. Point "a”, "b", and "c" were defined at 5\%, 10\%, and 15\% of failure strain and 6.15\%, 25\%, and 50\% of stiffness respectively. Point "d" and point "e" were assumed at $20 \%$ and $90 \%$ of the failure displacement (point f), respectively. (Modified from Ahn HS. A Virtual Model of the Human Cervical Spine for Physics-beased Simulation and Applications. Department of Biomedical Engineering. Memphis, Tennessee: The University of Tennessee Health Science Center, 2005.)

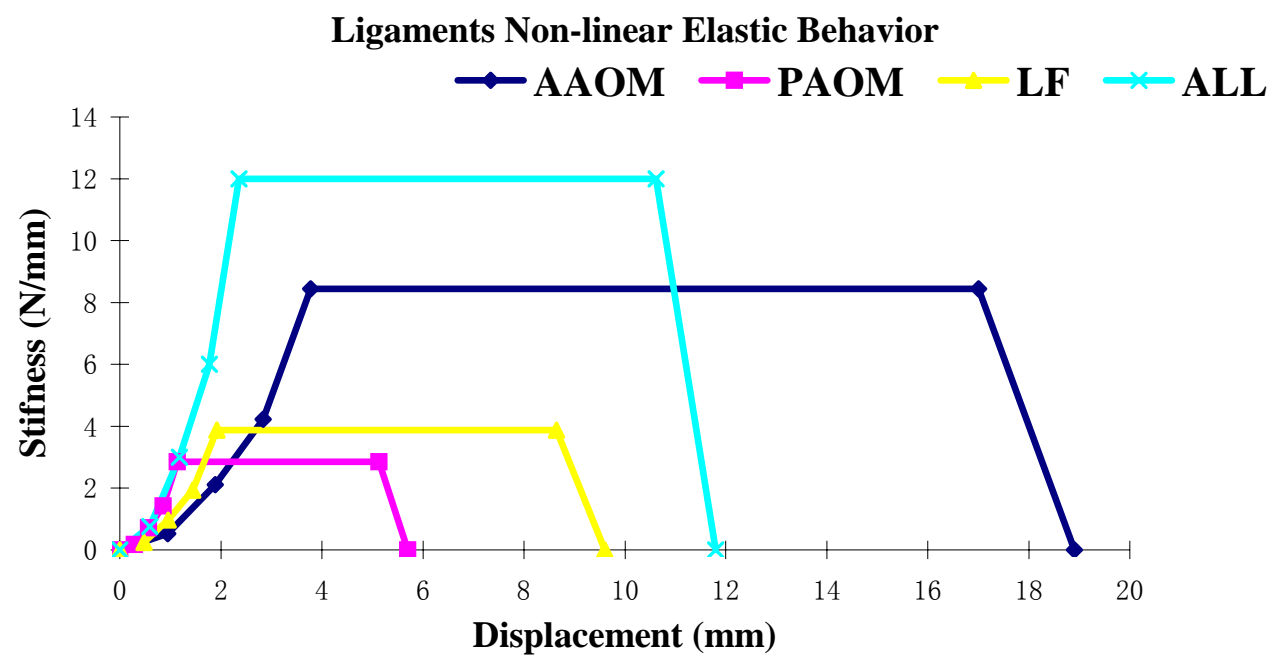

Figure 3-11: The stiffness-displacement curves plot based on Figure 3-10 for the ligaments in the upper cervical spine. 


\subsubsection{Muscles}

The complicated muscle system of the head and neck was represented by 19 distinct muscles, which were classified into 9 groups and modeled by 25 sub-volumes. In some neck muscle models, one bundle of muscle may be represented by more than one element to account for different points of attachment of the muscle. However, in this study, each muscle was modeled as one straight line element that was represented by an actuator in Visual Nastran 4D. Table 3-5 shows all the neck muscles developed in this muscle model and their attachment sites, which were defined according to published anatomic descriptions. ${ }^{2,11,18}$ The coordinates of the origins and insertions of the muscles represented an average position for the muscle attachment. Since T4 (the fourth thoracic body) and T5 (the fifth thoracic body) were not available in this model, the origin sites for the muscles splenius cervicis and longissimus cervicis were approximated.

According to the muscle motor functions, such as axial rotation, lateral bending, extension, and flexion, muscles were further categorized into a "Primary Muscle Group" for each movement (Table 3-6 and Table 3-7). As mentioned in Section (2.1.3), primary muscles causing axial rotation, lateral bending, extension, and flexion were called rotators, lateral flexors, extensors, and forward flexors, respectively. The motor functions were determined based on the classic text of Gray's Anatomy. ${ }^{34}$ 
Table 3-5: Neck muscle attachment sites.

\begin{tabular}{|c|c|c|}
\hline Muscle Name & Origin & Insertion \\
\hline \multicolumn{3}{|l|}{ Sternocleidomastoid } \\
\hline Sternomastoid & Sternum & Skull (m.p) \\
\hline Cleidomastoid & Clavicle & Skull (m.p) \\
\hline Cleido-occipital & Clavicle & Skull (s.n.l) \\
\hline \multicolumn{3}{|l|}{ Trapezius } \\
\hline Clavotapeius & Clavicle & Skull (m.p) \\
\hline Acromiotrapezius & Scapula & C7 (t.p) \\
\hline \multicolumn{3}{|l|}{ Sbocclpital } \\
\hline Rectus capitis posterior major & C2 (s.p.) & Skull (i.n.l) \\
\hline Rectus capitis posterior minor & C1 (p.t.) & Skull (i.n.l) \\
\hline Obliquus capitis superior & C1 (t.p.) & Skull (s.-i.n.l) \\
\hline Obliquus capitis inferior & C2 (s.p.) & C1( t.p.) \\
\hline \multicolumn{3}{|l|}{ Longus capitis and colli } \\
\hline Longus capitis & C4 (t.p.) & Skull (bas-occ) \\
\hline Longus colli-vetical & C3 ( ant v.b.) & C1 ( ant v.b.) \\
\hline Longus colli-superior oblique & C5 (t.p.) & C1 ( ant v.b \\
\hline Longus colli-inferior oblique & C3 ( ant v.b.) & C5 (t.p.) \\
\hline \multicolumn{3}{|l|}{ Splenius } \\
\hline Splenius capitis-medial & C6 (s.p.) & Skull (s.n.l.) \\
\hline Splenius capitis-lateral & T1 (s.p.) & Skull (m.p.) \\
\hline Splenius cervicis & T4 (s.p.) & C3 (s.p.) \\
\hline \multicolumn{3}{|l|}{ Semispinalis } \\
\hline Semispinalis capitis-lateral & C5 (a.p.) & Skull (s.-i.n.l) \\
\hline Semispinalis capitis-medial & T1 (t.p.) & Skull (s.-i.n.l) \\
\hline Semispinalis cervicis & T1 (t.p.) & C3 (s.p.) \\
\hline \multicolumn{3}{|l|}{ Scalenes } \\
\hline Scalenus anterior & Rib 1 & C4 (t.p.) \\
\hline Scalenus medius & Rib 1 & C3 (t.p.) \\
\hline Scalenus posterior & Rib 2 & C5 (t.p.) \\
\hline Levator scapulae & Scapula (med) & C2 (t.p.) \\
\hline \multicolumn{3}{|l|}{ Erector spinae } \\
\hline Longissimus capitis & C6 (a.p.) & Skull (i.n.l) \\
\hline Longissimus cervicis & T5 (t.p.) & C4 (t.p.) \\
\hline Iliocostalis cervicis & Rib 3 & C5 (t.p.) \\
\hline
\end{tabular}

Note: Modified from Vasavada AN, Li S, Delp SL. Influence of muscle morphometry and moment arms on the moment-generating capacity of human neck muscles. Spine 1998;23:412-22.
a.p. = articular process;
med $=$ medial border scapula,
p.t. = posterior tubercle;
acr. = acromion of scapula;
between spine and superior
ant v.b. = anterior vertebral
border;
body;
m.p. = mastoid process;
bas-occ = basi-occiput;
occ pr =external occipital
i.n.l. = inferior nuchal line;
protuberance;

$$
\begin{aligned}
& \text { s.-i.n.l = between superior and } \\
& \text { inferior nuchal lines; } \\
& \text { s.p. = spinous process; } \\
& \text { s.n.l. = superior nuchal line; } \\
& \text {.p. = transverse process. }
\end{aligned}
$$


Table 3-6: Primary muscles involved in axial rotation and lateral bending.

\begin{tabular}{|c|c|c|}
\hline Movement & Axial Rotation (Rotator) & Lateral Bending (Lateral Flexor) \\
\hline Muscles & $\begin{array}{l}\text { Sternocleidomastoid } \\
\text { Sternomastoid }(O) \\
\text { Cleidomastoid }(O) \\
\text { Cleido-occipital }(O) \\
\text { Trapezius } \\
\text { Clavotrapezius }(O) \\
\text { Acromiotrapezius }(O) \\
\text { Suboccipital } \\
\text { Rectus capitis posterior major (S) } \\
\text { Obliquus capitis inferior (S) } \\
\text { Longus capitis and colli } \\
\text { Longus colli-inferior oblique (O) } \\
\text { Splenius } \\
\text { Splenius capitis-medial (S) } \\
\text { Splenius capitis-lateral (S) } \\
\text { Splenius cervicis (S) } \\
\text { Semispinalis } \\
\text { Semispinalis capitis-lateral (S) } \\
\text { Semispinalis capitis-medial (S) } \\
\text { Semispinalis cervicis (S) } \\
\text { Scalenus } \\
\text { Scalenus anterior }(O) \\
\text { Erector Spinae } \\
\text { Longissimus capitis (S) }\end{array}$ & $\begin{array}{l}\text { Sternocleidomastoid } \\
\text { Sternomastoid (S) } \\
\text { Cleidomastoid (S) } \\
\text { Cleido-occipital (S) } \\
\text { Trapezius } \\
\text { Clavotrapezius (S) } \\
\text { Acromiotrapezius (S) } \\
\text { Longus capitis and colli } \\
\text { Longus colli-inferior oblique (O) } \\
\text { Longus colli-superior oblique (O) } \\
\text { Splenius } \\
\text { Splenius cervicis (S) } \\
\text { Scalenus } \\
\text { Scalenus anterior (O) } \\
\text { Scalenus medius (S) } \\
\text { Scalenus posterior (S) } \\
\text { Levator scapulae (S) } \\
\text { Erector spinae } \\
\text { Longissimus capitis (S) } \\
\text { Longissimus cervicis (S) } \\
\text { Iliocostalis cervicis (S) }\end{array}$ \\
\hline
\end{tabular}

Notes: The muscles in Italic are the ones involved in both axial rotation and lateral bending. "O" mean muscle rotates head in the opposite side to the muscles when contracting; "S" means muscles rotate head to the same side when shortening. 
Table 3-7: Primary muscles involved in extension and flexion.

\begin{tabular}{l|l|l}
\hline Movements & Extension (Extensor) & Flexion (Flexor) \\
\hline & Sternocleidomastoid & Sternocleidomastoid \\
cleidomastoid & Sternomastoid \\
& cleido-occipital & Longus capitis and colli \\
Trapezius & Longus capitis \\
& Clavotrapezius & longus colli-vetical \\
& Acromiotrapezius & longus colli-superior oblique \\
& Suboccipital & longus colli-inferior oblique \\
& Rectus capitis posterior major & Scalenus \\
& Rectus capitis posterior major & Scalenus anterior \\
& Obliquus capitis superior & Scalenus medius \\
& Splenius & Scalenus posterior \\
& Splenius capitis-medial & Levator scapulae \\
& Splenius capitis-lateral & \\
& Splenius cervicis & \\
& Semispinalis & \\
& Semispinalis capitis-lateral & \\
& Semispinalis capitis-medial & \\
& Semispinalis cervicis & \\
& Erector spinae & \\
& Longissimus capitis & \\
& Longissimus cervicis & \\
& Iliocostalis cervicis & \\
\hline
\end{tabular}




\subsection{Inverse dynamics}

\subsubsection{Method overview}

The application of inverse dynamics is widely used in biomechanics as a tool for motion analysis. ${ }^{33}$ Riener et al. ${ }^{33}$ concluded that inverse dynamic modeling can be an effective tool for motion analysis in patients with cerebellar disorders. Pedrocchi et al. ${ }^{29}$ applied inverse dynamics to investigate voluntary trunk movements during weightlessness. Vasavada et $a l .{ }^{40}$ investigated the effect of moment arms and morphometry on the moment-generating capacities of individual muscles by developing a biomechanical head-neck musculoskeletal model that represented cervical spine kinematics.

The objectives of this study were first to develop a human head-neck musculoskeletal system that could simulate axial rotation, lateral bending, flexion, and extension under the control of muscles, and then second to define and validate soft tissues properties. Since there are no muscle excitation functions available in our Biomechanics Laboratory or in the literature, neck muscle forces could not be calculated. The use of inverse dynamics method offered an alternative approach. A head-neck model driven under motion response was developed first. Its main function was to obtain muscle length changes over time. That data served as input date for the second model driven under muscle control. A general comparison of these two models is summarized in Table 3-8, and a schematic flow chart of the inverse dynamic method used in this study is presented in Figure 3-12. The detailed descriptions of the two models are provided in the following sections. 
Table 3-8: Comparison of motion-driven model and muscles-driven model.

\begin{tabular}{c|c|c}
\hline & Model Driven by Motion Response & Model Driven by Muscles \\
\hline Muscle & Force actuator (F=0) & $\begin{array}{c}\text { Length actuator (Length-time curve) } \\
\text { Force actuator (Inactive muscle: F=0) }\end{array}$ \\
\hline $\begin{array}{c}\text { Soft } \\
\text { Tissues }\end{array}$ & $\begin{array}{c}\text { No soft tissues } \\
\text { Motion driven joint }\end{array}$ & $\begin{array}{c}\text { Intervertebral discs, facet joints, } \\
\text { ligaments, C0-C1 and C1-C2 joints }\end{array}$ \\
\hline $\begin{array}{c}\text { Driving } \\
\text { Force }\end{array}$ & $\begin{array}{c}\text { Motion response obtained from literatures } \\
\text { and Ahn' models }\end{array}$ & $\begin{array}{c}\text { Muscle length changes over running time } \\
\text { obtained from the models driven by } \\
\text { motion response }\end{array}$ \\
\hline
\end{tabular}

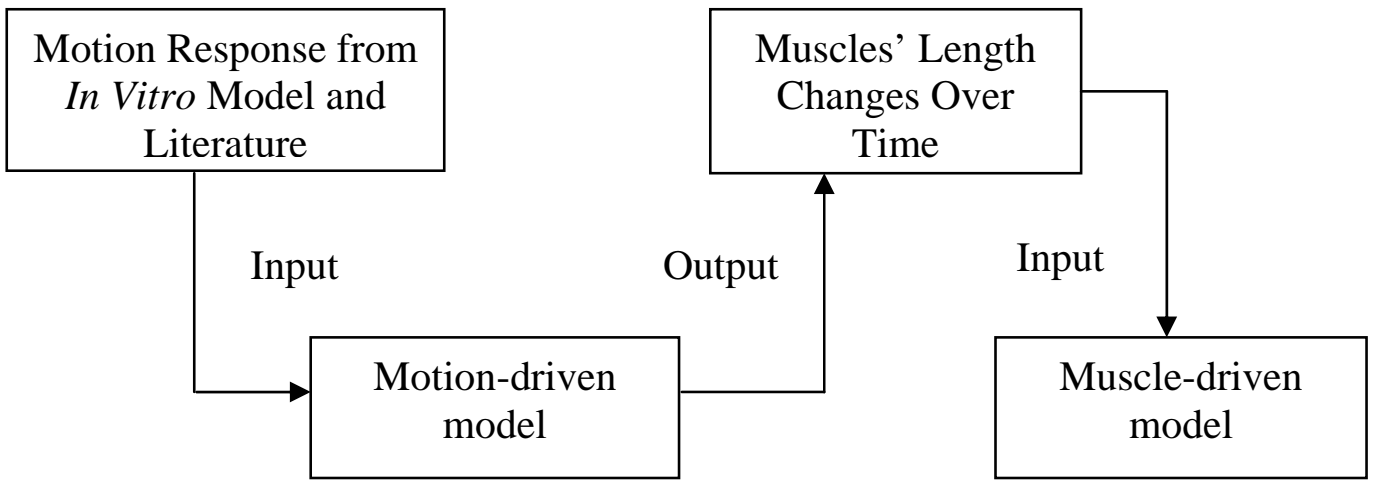

Figure 3-12: Flow chart of the inverse dynamic method. The input for the model driven by motion response was the motion response data from in vitro model and the literature. The output of the model driven by motion response was the muscle length-time relationship, which served as an input for the model driven by muscle length control. 


\subsubsection{Model driven by motion response}

In order to obtain the muscle length changes over time, a head-neck musculoskeletal model driven by motion responses was developed (Figure 3-13). Motion response was the driving force for this model, thus at each spinal level a motion driven joint, represented by a generic joint in Visual Nastran 4D, was developed. Further, each intervertebral joint between the C0 and T1 had three rotational degrees of freedom and three translational degrees of freedom. Therefore, motion driven joints were placed at each motion segment unit (C0-T1) and replaced all soft tissues, including intervertebral discs (C2-T1), C0-C1-C2 complex (C0-C2), facet joints, and ligaments. All motion driven joints from C2 to C7 were positioned at the exact same location as the native discs in the lower cervical spine. Joints C0-C1 and C1-C2, defined above, were

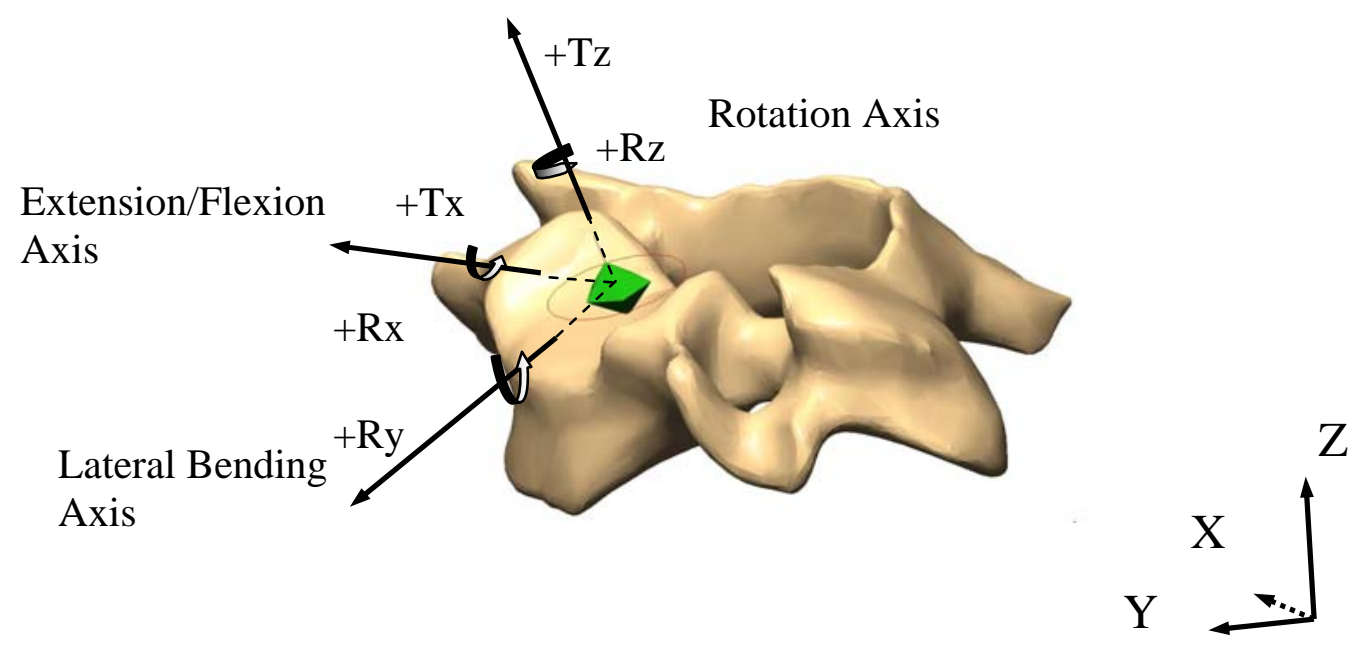

Figure 3-13: The three dimensional coordinate system for the motion driven joints. 
replaced by motion driven joints that similar to the intervertebral discs in the lower region, although no discs were present in the native upper cervical spine region. The $\mathrm{Xd}, \mathrm{Yd}$, and $\mathrm{Zd}$ coordinate axes were defined pointing to the right-laterally, anteriorly, and cranially, respectively (Figure 3-13).

Displacement-time and rotation-time curves were applied to each generic joint. $\mathrm{Ahn}^{3}$ developed a multi-body model of human cervical spine (C2-T1) and built a virtual laboratory simulator for kinematic and kinetic testing of the models. From the simulation results, the contributions of the individual motion segment rotations to the global rotation were comparable to the in vivo data from White and Panjabi. ${ }^{27,41}$ Therefore, translational data $(\mathrm{X}, \mathrm{Y}, \mathrm{Z})$ and rotational data $(\mathrm{Rx}, \mathrm{Ry}, \mathrm{Rz})$ of each joint in the lower cervical spine were adapted and modified from the kinematics output of Ahn's in vitro models.

However, similar detailed information for the upper cervical spine was not available in Ahn's in vitro model. As such, the upper spinal motion driven joints were modeled as motionbased joints that allowed Rx- rotation, Ry- rotation, Rz- rotation in flexion/extension, one-side lateral bending, and axial rotation, respectively. Intervertebral rotations were specified as a percentage of the total rotation, and the motions for level C0-C1 and C1-C2 were scaled from the “representative values” of intervertebral motions reported by White and Panjabi. ${ }^{27,41}$

Translations of the upper vertebrae were so small, they were considered negligible. No coupling behaviors were included here.

The aim of motion-driven model was to obtain the changes in muscle length over time. Force actuators were chosen to represent muscles in Visual Nastran 4D. The force resistance of the actuator was set to $0 \mathrm{~N}$ manually and the length changes of each "muscle" (actuator) was recorded by the length meter in Visual Nastran 4D. 


\subsubsection{Musculoskeletal head-neck system driven under muscle control}

The description of the dynamic cervical spine model was provided in the Section (3.1). Motion driven joints in the motion-driven model were replaced with the intervertebral discs, facet joints, ligaments, and joints $\mathrm{C} 0-\mathrm{C} 1$ and $\mathrm{C} 1-\mathrm{C} 2$ to accomplish this task. Compared to other regions of the human musculoskeletal system, the head-neck region is one of the more complex structures to computationally model because of the many muscles attached between them. Different combinations of neck muscles used to drive this musculoskeletal model may produce different modeling results. However, in order to perform a computer simulation and numerical optimization of the cervical spine dynamics within a reasonable computational run time, it was necessary to limit the complexity of the model. ${ }^{7}$ One of the key methods to reduce the complexity of this model in this study was to focus on the major muscles related to each movement. Therefore, for each specific movement, only the primary muscles involved in the movement were considered.

A concentric contraction is defined as a contraction causing movement when the muscle is shortening. An eccentric contraction is when the muscle lengthens under tension which controls movement caused by other muscles or gravity. One-side axial rotation and one-side lateral bending were caused by the single-side muscle contractions and were assumed to represent a concentric contraction in this study. Muscle lengthening activities provide a resistive force that tries to maintain the head and neck in the neutral position. In extension, the extensor muscles contract concentrically, moving the neck to the same side, while the anterior forward flexor muscles help to stabilize the spine. A similar action occurs in flexion, but in that instance the forward flexor muscles flex the head forward instead. 
The vertebral body was actuated by three different sets of muscles to construct the "All muscles" mode, the "Concentric contraction muscles only" mode, and the "Eccentric contraction muscles only" mode. The eccentric muscle activity during axial rotation can be referred to as "Passive elongation muscles only”, as the lengthening muscles act passively during this movement. Within Visual Nastran 4D, the muscles were categorized into eccentric contraction muscles and concentric contraction muscles mainly based on the trend of the muscle length changes. If the muscle length decreased over time, it belonged to the concentric contraction muscles; otherwise, it was considered as a member of eccentric contraction muscles. Table 3-9 shows the behavior of the muscles in these three study modes for each movement. As mentioned in Section (3.2.1), muscles in the motion-driven model were represented by force actuators in Visual Nastran 4D, while in the muscle-driven model, activated muscles were modeled as displacement actuators. The muscle length-time relationship for an individual muscle was obtained from the motion-driven model and became the input data for the muscle-driven model.

\subsection{Model evaluation}

Published in vivo data from White and Panjabi ${ }^{41}$ were used to validate motion-driven and muscle-driven models. The contribution of each motion segment unit rotation relative to the overall global motion of cervical spine was compared between the simulation model (both the model driven by motion response and model driven under muscle control) and the in vivo data ${ }^{41}$ and served as the model evaluation step. 
Table 3-9: Muscle behaviors in the three study modes.

\begin{tabular}{|c|c|c|c|}
\hline & Movement & Muscles & $\begin{array}{c}\text { Muscles (Actuator) } \\
\text { in VN 4D }\end{array}$ \\
\hline \multirow{4}{*}{$\begin{array}{c}\text { “All Muscles" } \\
\text { Mode }\end{array}$} & Axial rotation & $\begin{array}{c}\text { Both sides of the paired } \\
\text { muscles }\end{array}$ & \multirow{4}{*}{$\begin{array}{c}\text { Some are lengthening, } \\
\text { some are shortening }\end{array}$} \\
\hline & Lateral Bending & $\begin{array}{c}\text { Both sides of the paired } \\
\text { muscles }\end{array}$ & \\
\hline & Extension & Extensor+Flexor muscles & \\
\hline & Flexion & Extensor+Flexor muscles & \\
\hline \multirow{4}{*}{$\begin{array}{c}\text { “Eccentric } \\
\text { Contraction } \\
\text { Muscles Only" } \\
\text { Mode (Passive } \\
\text { Elongation } \\
\text { Muscles Only" } \\
\text { mode for Axial } \\
\text { Rotation) }\end{array}$} & Axial rotation & $\begin{array}{c}\text { Only one side of paired } \\
\text { the muscles }\end{array}$ & \multirow{4}{*}{ Lengthening } \\
\hline & Lateral Bending & $\begin{array}{l}\text { Only one side of paired } \\
\text { the muscles }\end{array}$ & \\
\hline & Extension & Flexor muscles, paired & \\
\hline & Flexion & Extensor muscles, paired & \\
\hline \multirow{4}{*}{$\begin{array}{c}\text { “Concentric } \\
\text { Contraction } \\
\text { Muscles Only" } \\
\text { Mode }\end{array}$} & Axial rotation & $\begin{array}{l}\text { Only one side of the } \\
\text { paired muscles }\end{array}$ & \multirow{4}{*}{ Shortening } \\
\hline & Lateral Bending & $\begin{array}{l}\text { Only one side of the } \\
\text { paired muscles }\end{array}$ & \\
\hline & Extension & Extensor muscle, paired & \\
\hline & Flexion & Flexor muscle, paired & \\
\hline
\end{tabular}




\section{Results}

This chapter consists of three parts: a musculoskeletal head-neck model is represented in Section (4.1), the results from the model driven by motion response are discussed in Section (4.2), and the results from the model driven by muscle length are provided in Section (4.3).

\subsection{Simulation of musculoskeletal head-neck complex}

A musculoskeletal head-neck model that simulated in vivo movements was developed. The model consisted of the skull, cervical and thoracic vertebrae, interconnecting joints, ligaments, and nine groups of head-neck muscles. The structure of the skull and vertebrae in the model were shown in Figure 3-1. The structure of the interconnecting joints and ligaments in the model are demonstrated in Figure 3-5. A detailed description of the origin and insertion of each muscle element is provided in Appendix A. Illustration of the frontal, lateral, and rear views of the final model are shown in Figure 4-1.

\subsection{Motion-driven model results}

Two sets of simulation results were generated from the head-neck model driven under motion control. The first data set represented the percent contribution of each motion segment unit to the overall global response for axial rotation, lateral bending, extension, and flexion. The 


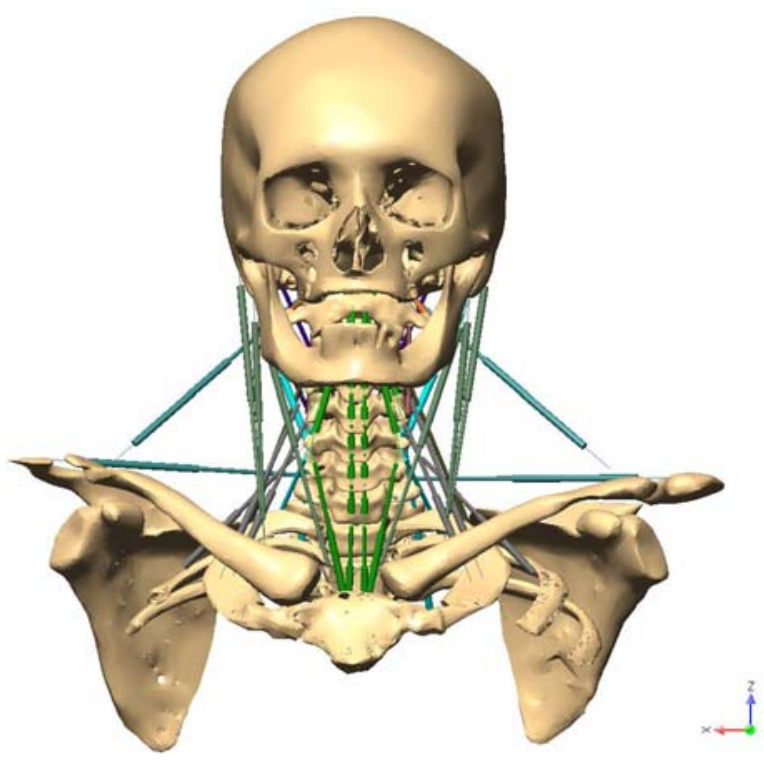

(A)

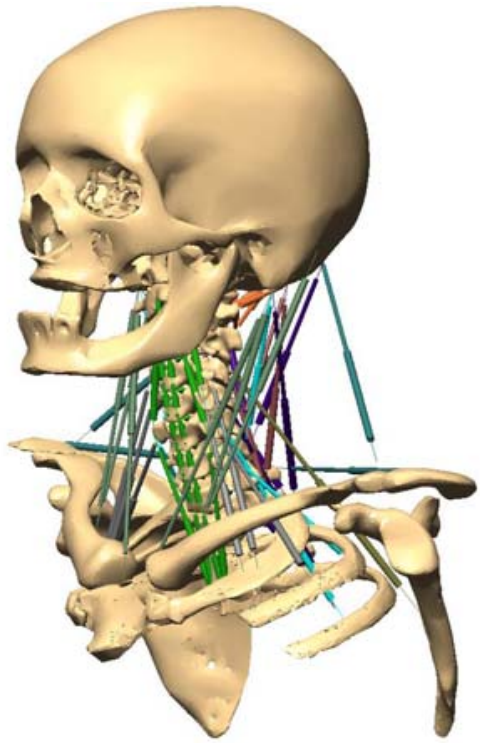

(B)

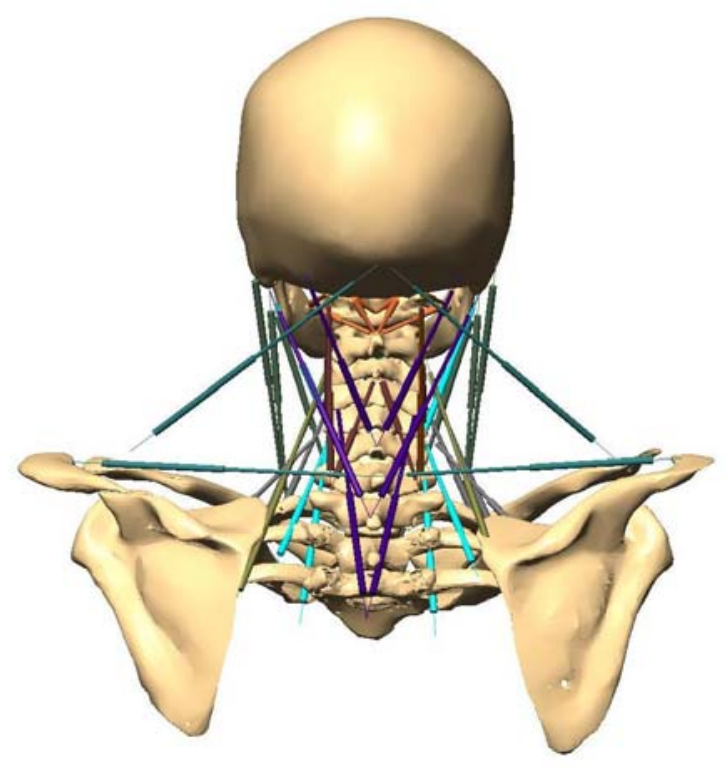

(C)

Figure 4-1: Musculoskeletal head-neck simulation model. (A) Frontal view. (B) Lateral view. (C) Rear view. 
second data set represented the change in length of each muscle over time for different movements. This information was used as input data to drive the muscle-driven model.

\subsubsection{Evaluation of motion-driven model}

The motion segment unit rotations, expressed as a percent of the global rotation, are shown in Figure 4-2 for the motion-driven model and published in vivo data ${ }^{41}$. There was a close match between the two data sets for all head movements.

\subsubsection{Muscle length-time relationship}

The most important result from the model driven under motion control was the lengthtime curve for each muscle in one-side axial rotation, one-side lateral bending, flexion, and extension. During one-side axial rotation and one-side lateral bending, the muscles on one side of the paired primary muscles shorten while the other side lengthens, indicating muscles in these two movements work alone to rotate or bend the head and neck obliquely. However, the paired muscles lengthen or shorten simultaneously, acting together to complete the motion during flexion and extension. 

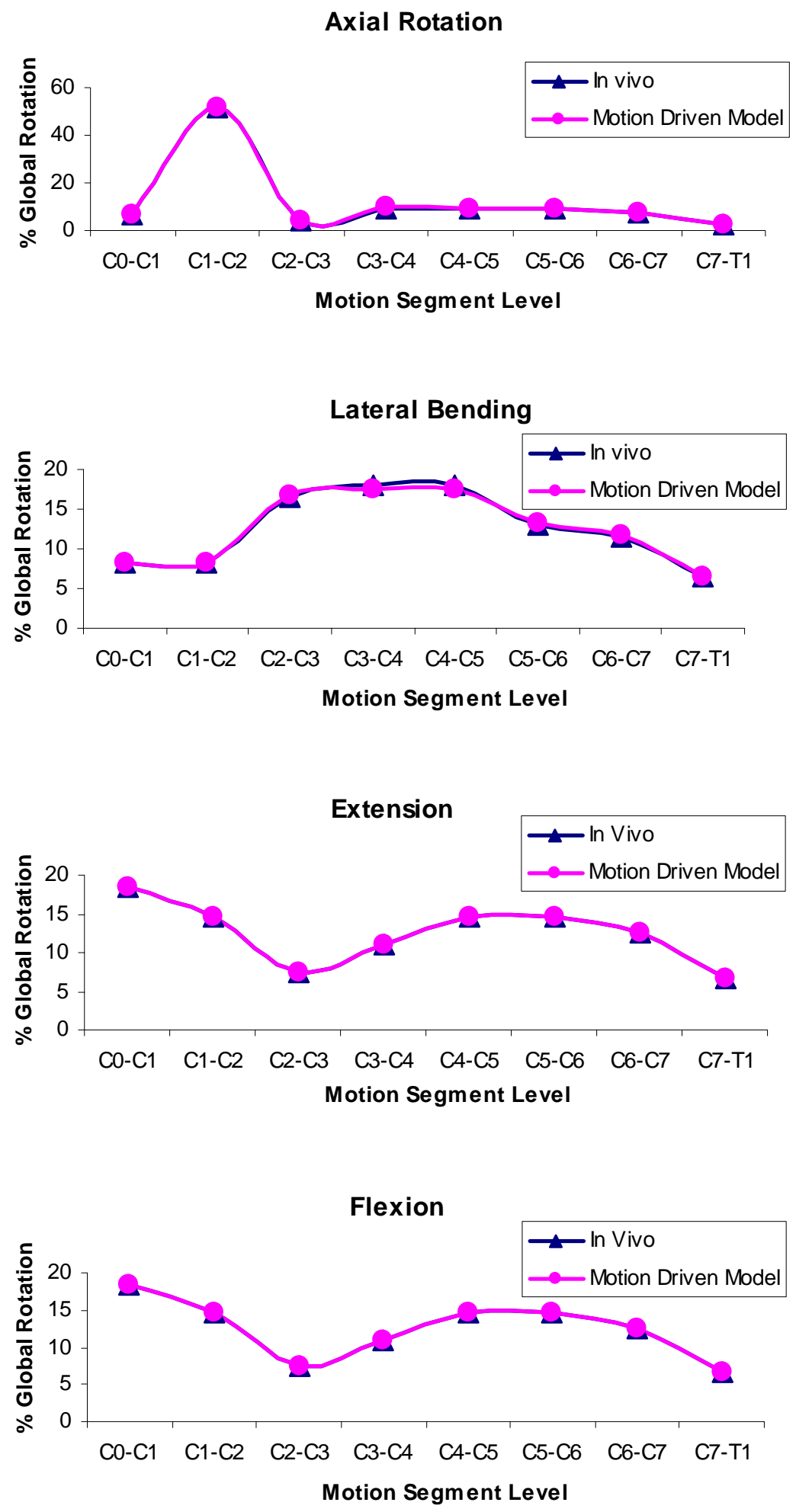

Figure 4-2: Comparisons of the segment unit rotations between the simulation model driven under motion control and in vivo data for different movements. (A) Axial rotation, (B) One side lateral bending, (C) Extension, and (D) Flexion. 


\subsection{Muscle driven model results}

The simulation results were divided into four parts according to the four types of movements: axial rotation, lateral bending, flexion, and extension. For each specific movement, muscle selections for the three different modes: "All muscles” mode, "Concentric contraction muscles only" mode, and "Eccentric contraction muscles only" mode, were based on the muscle length-time relationship obtained from the motion-driven model. The "All muscles" mode included all the primary muscles involved in the specific movement; "Concentric contraction muscles only" mode only included those muscles shortened with time; while "Eccentric contraction muscles only mode" only included those muscles lengthened with time. The motion segment unit rotations for each type of movement tested under each mode were plotted against the corresponding run time. Figure 4-3 shows the typical curves of the rotation versus time for each segment level under the "All muscles" mode in axial rotation. The remainder curves are provided in Appendix B.

\subsubsection{Axial rotation}

For the axial rotation movement under the "All muscles" mode, all of the primary muscles involved in axial rotation (rotator) were chosen. Under the "Concentric contraction muscles only” mode (Figure 4-4), only those muscles that shortened with time were considered (Figure 4-5), while under the "Passive elongation muscles only" mode, only those muscles that 


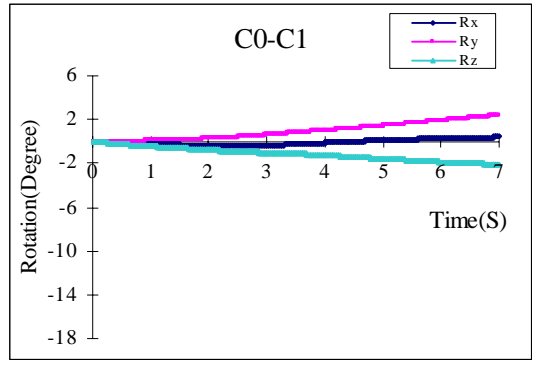

(A)

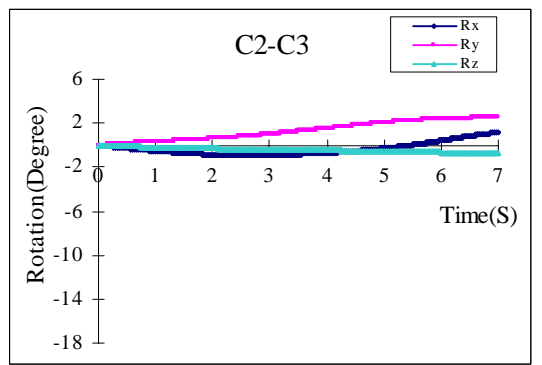

(C)

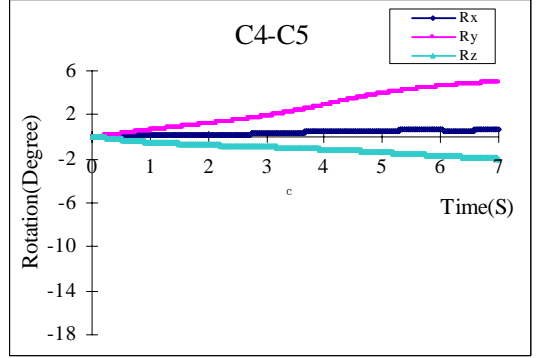

(E)

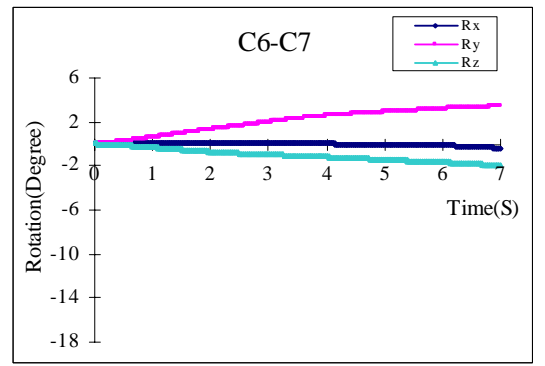

(G)

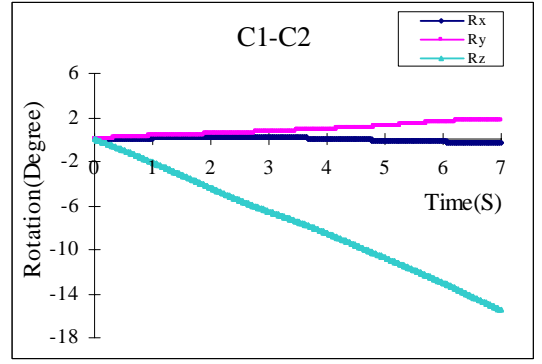

(B)

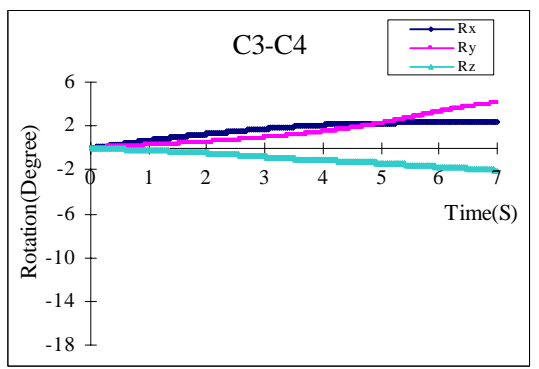

(D)

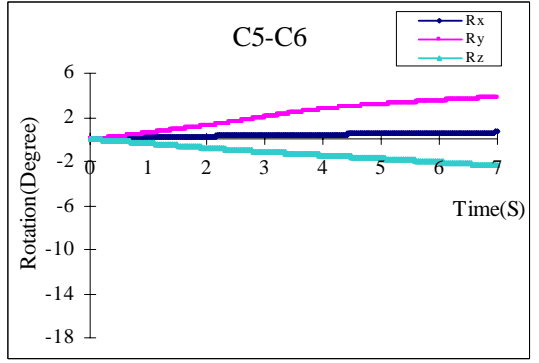

(F)

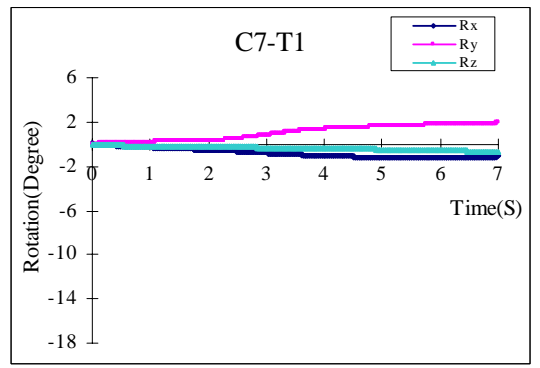

$(\mathrm{H})$

Figure 4-3: The rotation versus time curves for each segment level under the "All muscles" mode in axial rotation. (A) C0-C1. (B) C1-C2. (C) C2-C3. (D) C3-C4. (E) C4-C5. (F). C5-C6. (G) C6-C7. (H) C7-T1. 


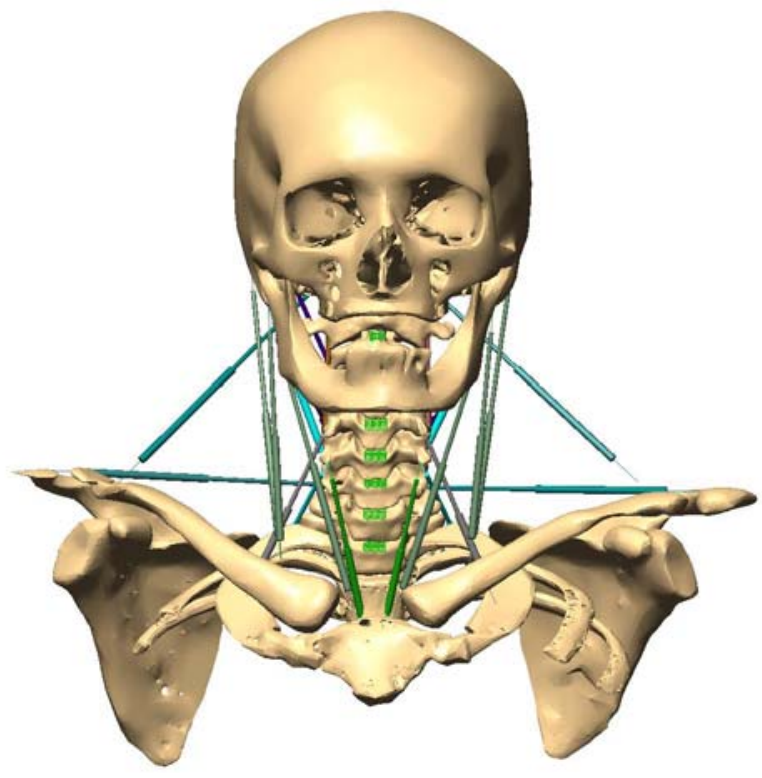

(A)

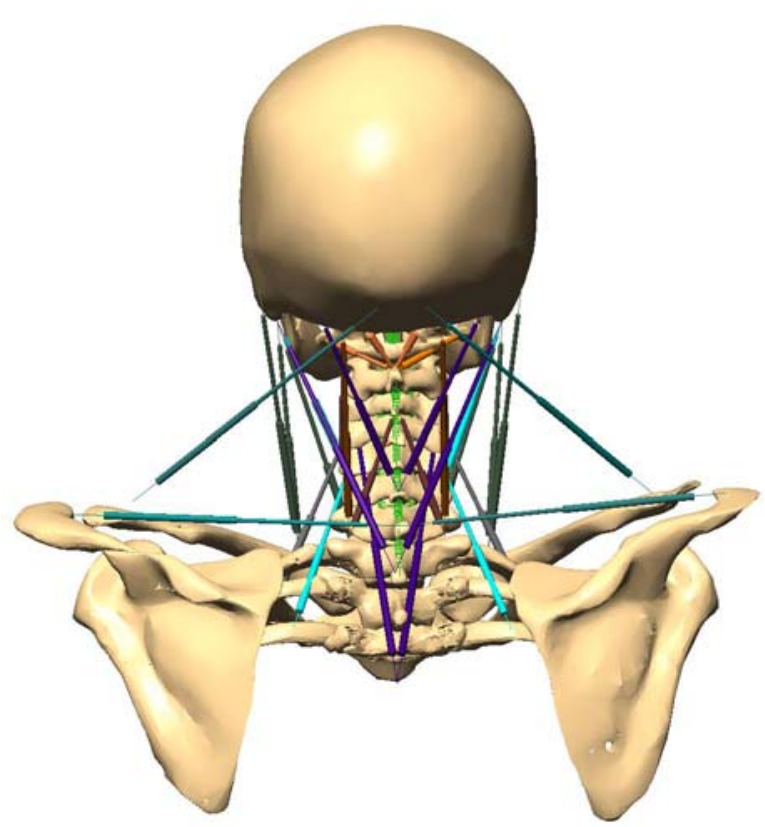

(B)

Figure 4-4: Musculoskeletal head-neck model under “All muscles” mode during right-side axial rotation. (A) Frontal view. (B) Rear view. 


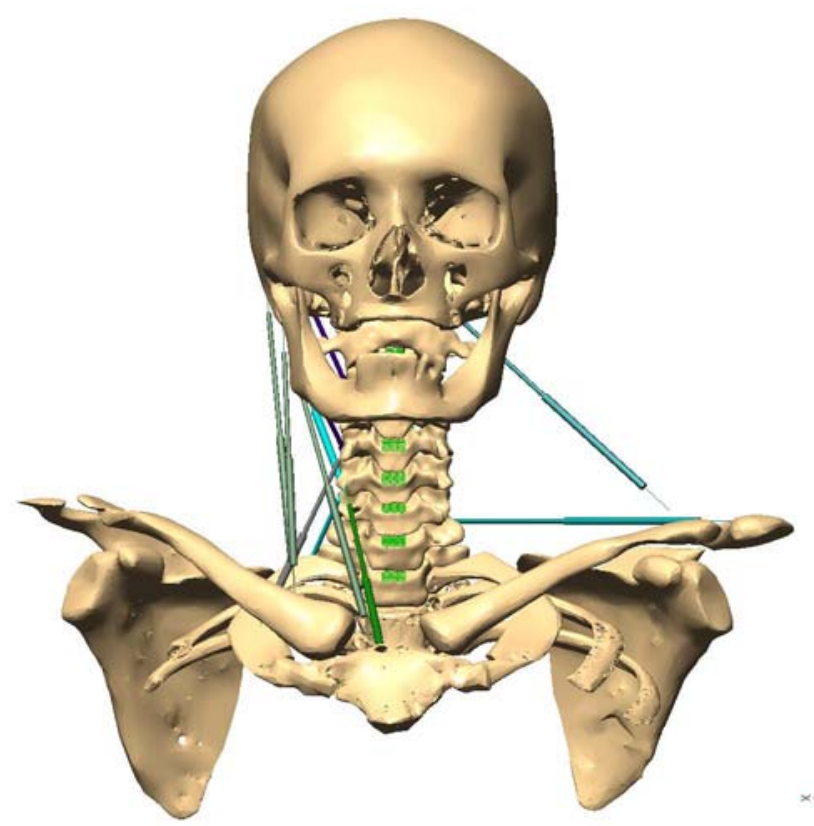

(A)

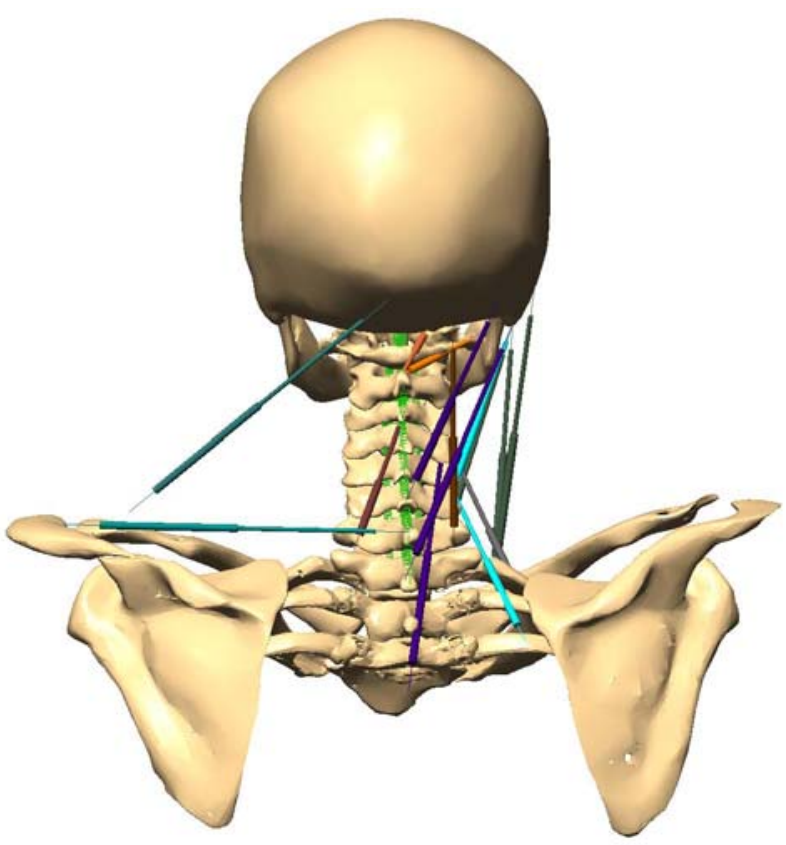

(B)

Figure 4-5: Musculoskeletal head-neck model under “Concentric contraction muscles only" mode during right-side axial rotation. (A) Frontal view. (B) Rear view. 
lengthened were activated (Figure 4-6). Muscle selection for each study mode are listed in Table 4-1. The distribution of relative motion segment unit rotations normalized to the global (C0-T1) rotation during axial rotation is shown in Figure 4-7 for different muscle activation modes and in vivo data. The simulation results of all the three modes were comparable to the in vivo data. The contributions of C1-C2 segment to the overall global rotation in the "Concentric contraction muscles only" mode and the "Eccentric contraction muscles only" mode were $61 \%$ and 62\%, respectively, compared to the in vivo data value of 52\%. The contribution from the "All muscles” mode was the closest at 57\%.

\subsubsection{Lateral bending}

The muscle selection for the three muscle activation modes used for lateral bending was similar to the ones used to study axial rotation. Under the “All muscles” mode, all the primary muscles involved in lateral bending (lateral flexor muscles) were chosen (Figure 4-8). Under the “Concentric concentration muscles only” mode (Figure 4-9), only those muscles that shortened in length with time were considered. Lastly, for the "Eccentric contraction muscles only mode”, only those muscles that lengthened were activated (Figure 4-10). Muscle selection for each study mode is listed in Table 4-2.

The distribution of relative motion segment unit rotations normalized to the global (C0T1) rotation during lateral bending is shown in Figure 4-11 for the different muscle activation modes and in vivo data. The results from “All muscles” mode had the closest fit with in vivo 


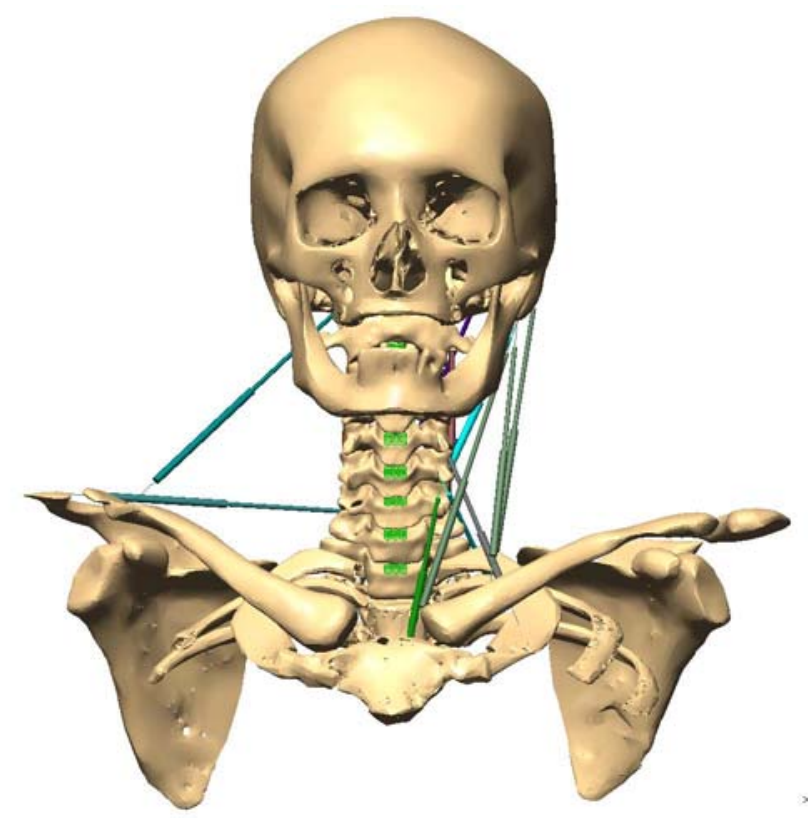

(A)

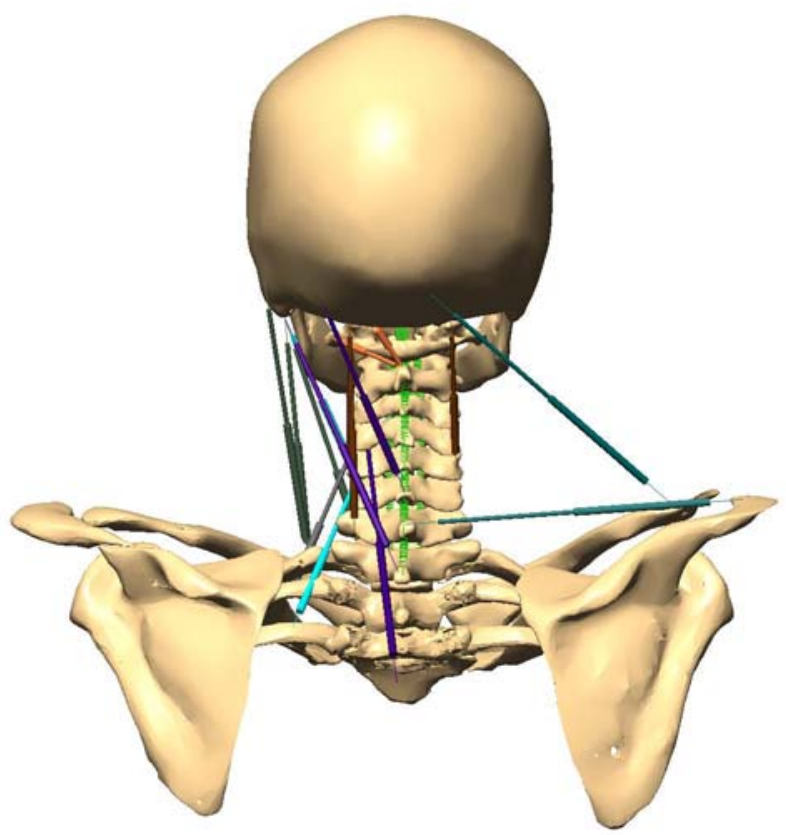

(B)

Figure 4-6: Musculoskeletal head-neck model under "Passive elongation muscles only" mode during right-side axial rotation. (A) Frontal view. (B) Rear view. 
Table 4-1: Muscle associated with each muscle activation mode in right-side axial rotation.

\begin{tabular}{|c|c|c|c|}
\hline Muscles & 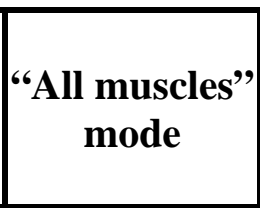 & \begin{tabular}{|c|} 
“Concentric \\
contraction \\
muscles \\
only” mode
\end{tabular} & \begin{tabular}{|c|} 
"Eccentric \\
contraction \\
muscles only" \\
mode
\end{tabular} \\
\hline \multicolumn{4}{|l|}{ Sternocleidomastoid } \\
\hline Sternomastoid & B & $\mathrm{R}$ & $\mathrm{L}$ \\
\hline Cleidomastoid & B & $\mathrm{R}$ & $\mathrm{L}$ \\
\hline Cleido-occipital & B & $\mathrm{R}$ & $\mathrm{L}$ \\
\hline \multicolumn{4}{|l|}{ Trapezius } \\
\hline Clavotrapezius & B & $\mathrm{L}$ & $\mathrm{R}$ \\
\hline Acromiotrapezius & B & $\mathrm{L}$ & $\mathrm{R}$ \\
\hline \multicolumn{4}{|l|}{ Suboccipital } \\
\hline Rectus capitis posterior major & B & $\mathrm{R}$ & $\mathrm{L}$ \\
\hline Obliquus capitis inferior & B & $\mathrm{R}$ & $\mathrm{L}$ \\
\hline \multicolumn{4}{|l|}{ Longus capitis and colli } \\
\hline Longus colli-inferior oblique & B & $\mathrm{R}$ & $\mathrm{L}$ \\
\hline \multicolumn{4}{|l|}{ Splenius } \\
\hline Splenius capitis-medial & B & $\mathrm{R}$ & $\mathrm{L}$ \\
\hline Splenius capitis-lateral & B & $\mathrm{R}$ & $\mathrm{L}$ \\
\hline Splenius cervicis & B & $\mathrm{R}$ & $\mathrm{L}$ \\
\hline \multicolumn{4}{|l|}{ Semispinalis } \\
\hline Semispinalis capitis-lateral & B & $\mathrm{R}$ & $\mathrm{L}$ \\
\hline Semispinalis capitis-medial & B & $\mathrm{R}$ & $\mathrm{L}$ \\
\hline Semispinalis cervicis & B & $\mathrm{R}$ & $\mathrm{L}$ \\
\hline \multicolumn{4}{|l|}{ Scalenus } \\
\hline Scalenus anterior & B & $\mathrm{R}$ & $\mathrm{L}$ \\
\hline \multicolumn{4}{|l|}{ Erector Spinae } \\
\hline Longissimus capitis & B & $\mathrm{R}$ & $\mathrm{L}$ \\
\hline
\end{tabular}

Note: B, R, L represents both-side, right-side, and left-side muscles were activated, respectively. 


\section{Axial Rotation}

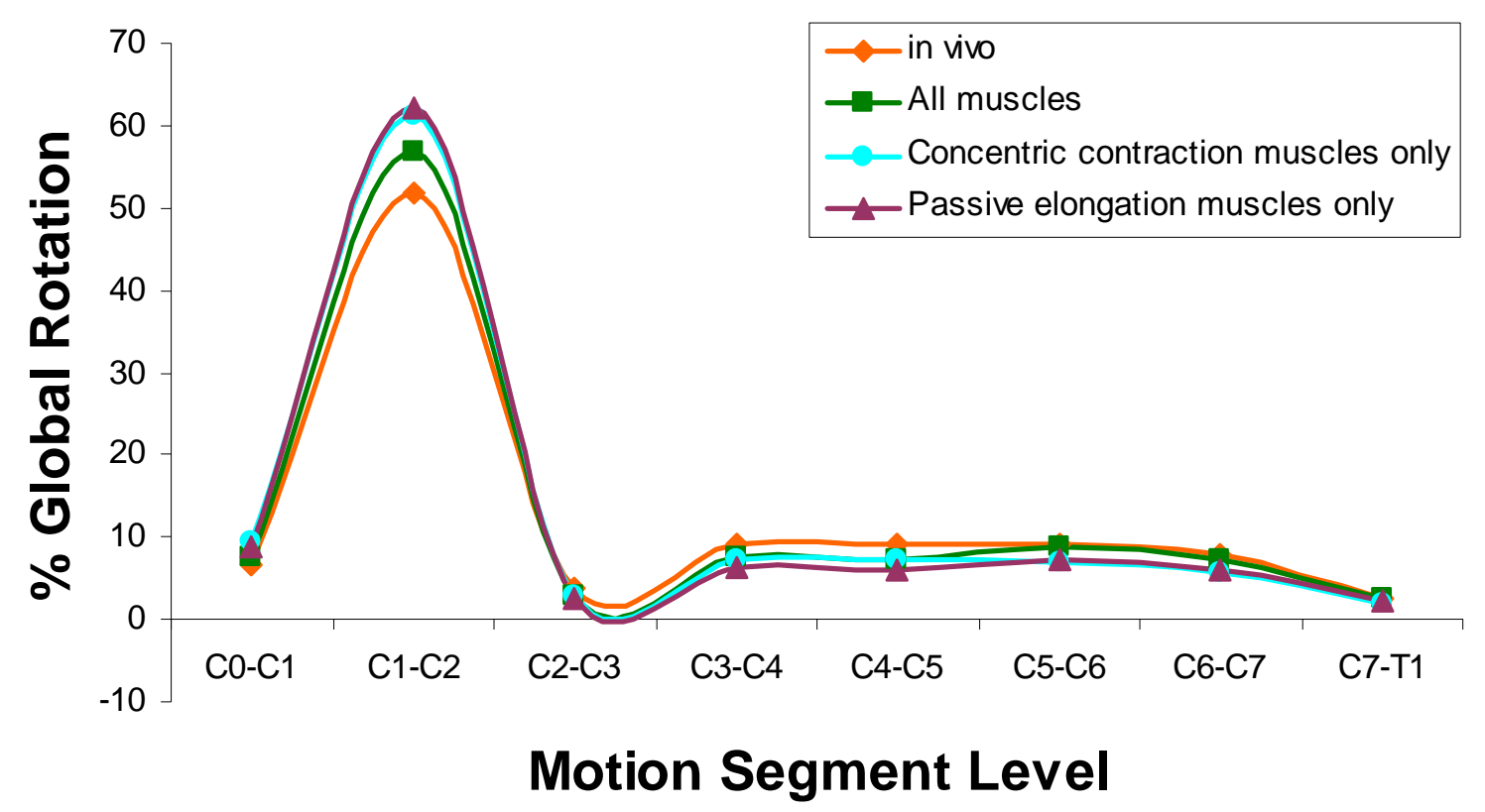

Figure 4-7: Comparisons of the distribution of relative motion segment unit rotations during axial rotation for the "All muscles" mode, "Concentric contraction muscles only" mode, "Passive elongation muscles only" mode, and in vivo data. 


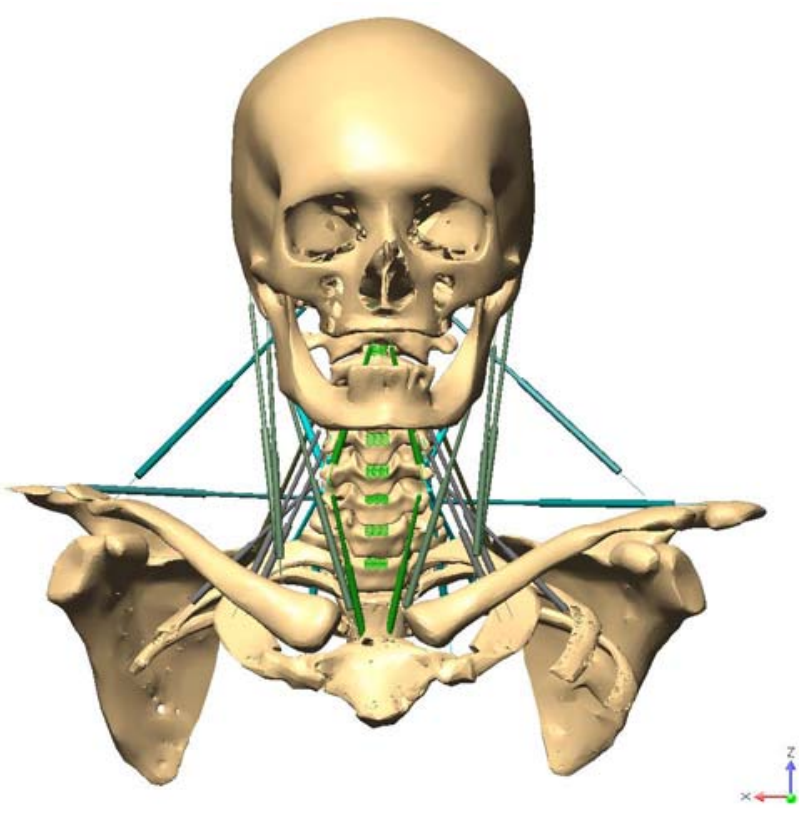

(A)

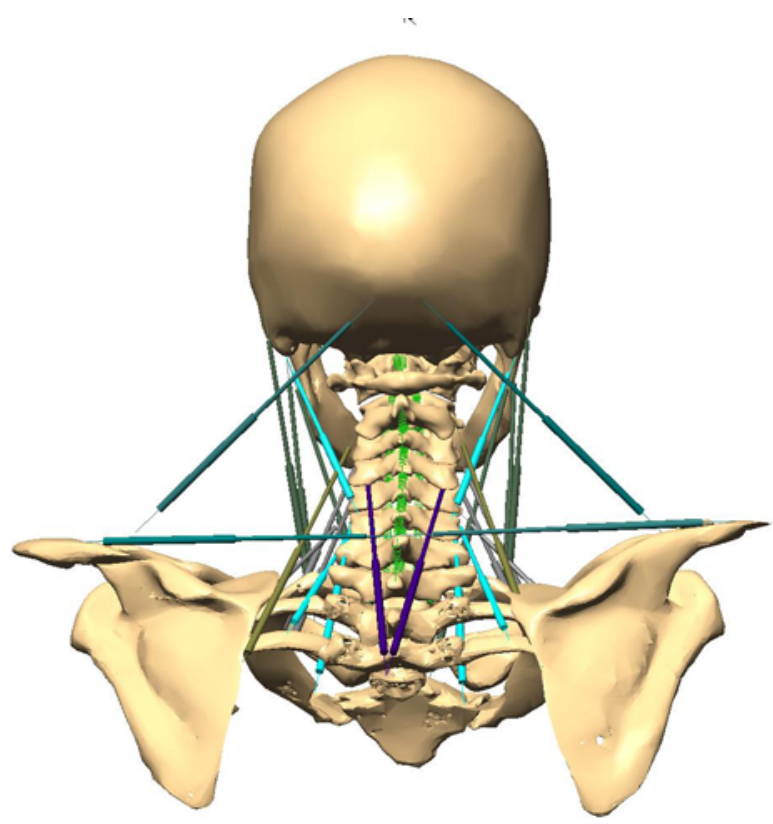

(B)

Figure 4-8: Musculoskeletal head-neck model under "All muscles" mode during left-side lateral bending. (A) Frontal view. (B) Rear view. 


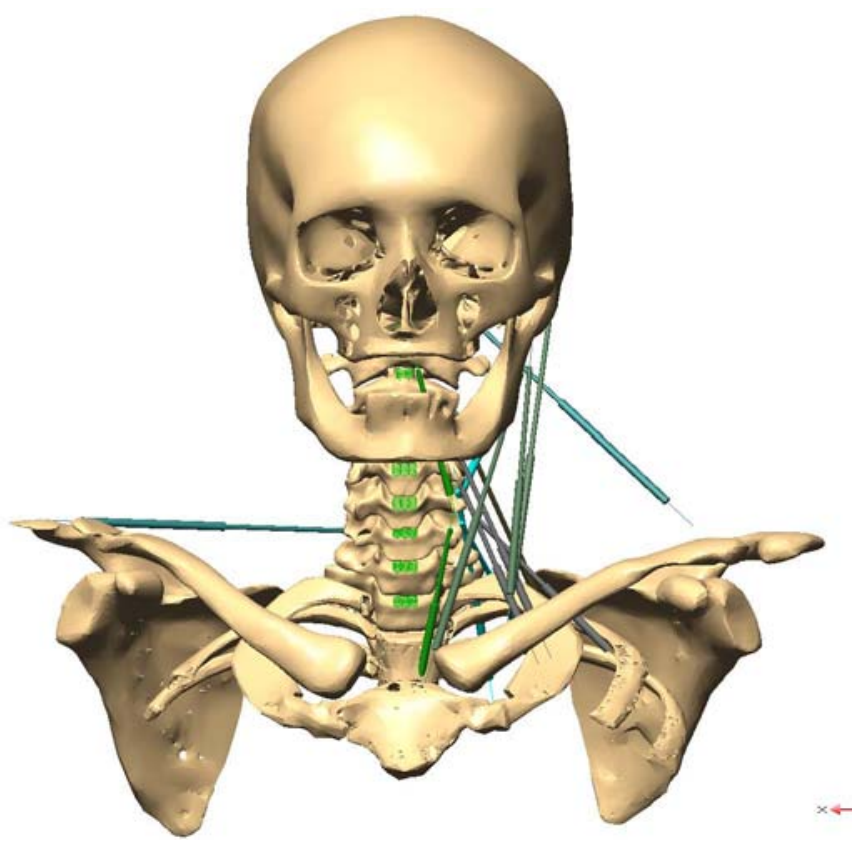

(A)

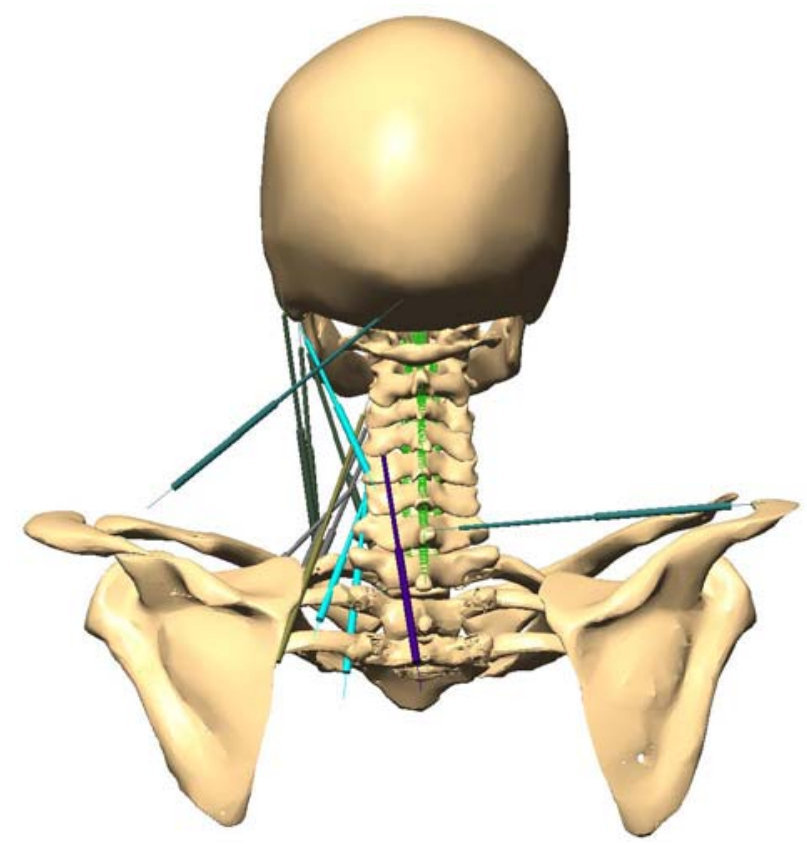

(B)

Figure 4-9: Musculoskeletal head-neck model under "Concentric contraction muscles only" mode during left-side lateral bending. (A) Frontal view. (B) Rear view. 


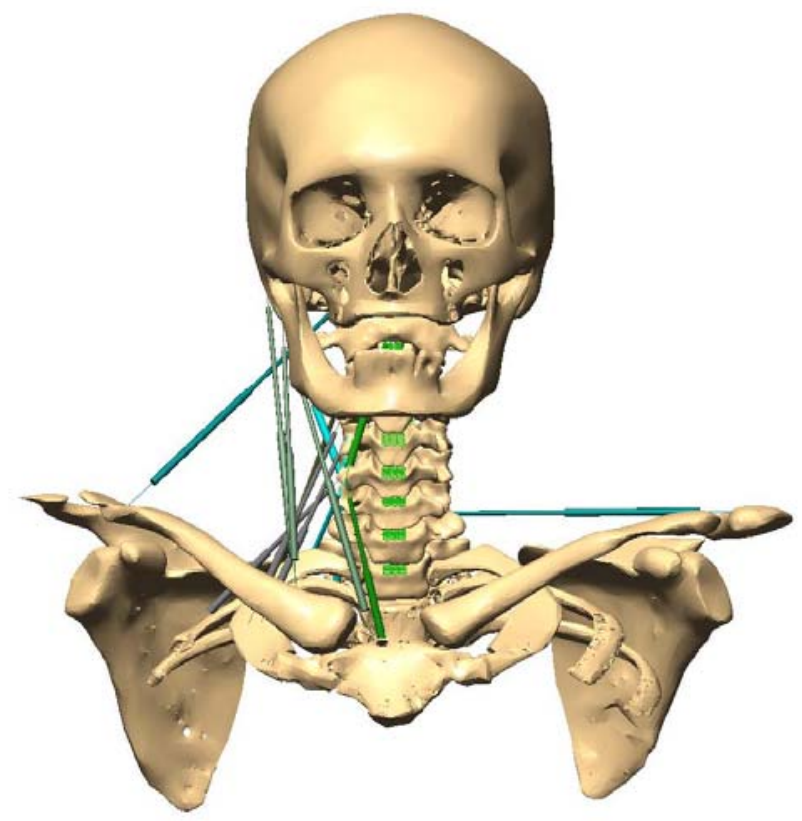

(A)

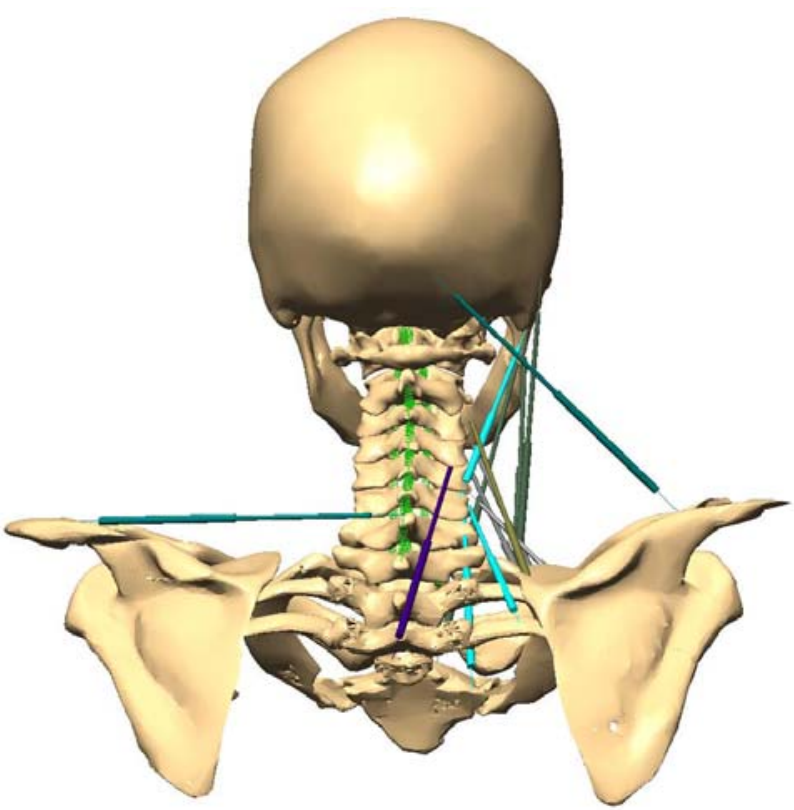

(B)

Figure 4-10: Musculoskeletal head-neck model under "Eccentric contraction muscles only" mode during left-side lateral bending. (A) Frontal view. (B) Rear view. 
Table 4-2: Muscles associated with each muscle activation mode in left-side lateral bending.

\begin{tabular}{|c|c|c|c|}
\hline Muscles & $\begin{array}{c}\text { "All } \\
\text { muscles" } \\
\text { mode }\end{array}$ & $\begin{array}{c}\text { “Concentric } \\
\text { contraction } \\
\text { muscles } \\
\text { only” mode }\end{array}$ & $\begin{array}{c}\text { "Eccentric } \\
\text { contraction } \\
\text { muscles } \\
\text { only" mode }\end{array}$ \\
\hline \multicolumn{4}{|l|}{ Sternocleidomastoid } \\
\hline Sternomastoid & B & $\mathrm{L}$ & $\mathrm{R}$ \\
\hline Cleidomastoid & B & $\mathrm{L}$ & $\mathrm{R}$ \\
\hline Cleido-occipital & B & $\mathrm{L}$ & $\mathrm{R}$ \\
\hline \multicolumn{4}{|l|}{ Trapezius } \\
\hline Clavotrapezius & B & $\mathrm{L}$ & $\mathrm{R}$ \\
\hline Acromiotrapezius & B & $\mathrm{R}$ & $\mathrm{L}$ \\
\hline \multicolumn{4}{|l|}{ Longus capitis and colli } \\
\hline Longus colli-inferior oblique & B & $\mathrm{L}$ & $\mathrm{R}$ \\
\hline Longus colli-superior oblique & B & $\mathrm{L}$ & $\mathrm{R}$ \\
\hline \multicolumn{4}{|l|}{ Splenius } \\
\hline Splenius cervicis & B & $\mathrm{L}$ & $\mathrm{R}$ \\
\hline \multicolumn{4}{|l|}{ Scalenus } \\
\hline Scalenus anterior & B & $\mathrm{L}$ & $\mathrm{R}$ \\
\hline Scalenus medius & B & $\mathrm{L}$ & $\mathrm{R}$ \\
\hline Scalenus posterior & B & $\mathrm{L}$ & $\mathrm{R}$ \\
\hline Levator scapulae & B & $\mathbf{L}$ & $\mathbf{R}$ \\
\hline \multicolumn{4}{|l|}{ Erector spinae } \\
\hline Longissimus capitis & B & $\mathrm{L}$ & $\mathrm{R}$ \\
\hline Longissimus cervicis & B & $\mathrm{L}$ & $\mathrm{R}$ \\
\hline Iliocostalis cervicis & B & $\mathrm{L}$ & $\mathrm{R}$ \\
\hline
\end{tabular}

Note: B, R, L represents both-side, right-side, and left-side muscles were activated, respectively. 


\section{Lateral Bending}

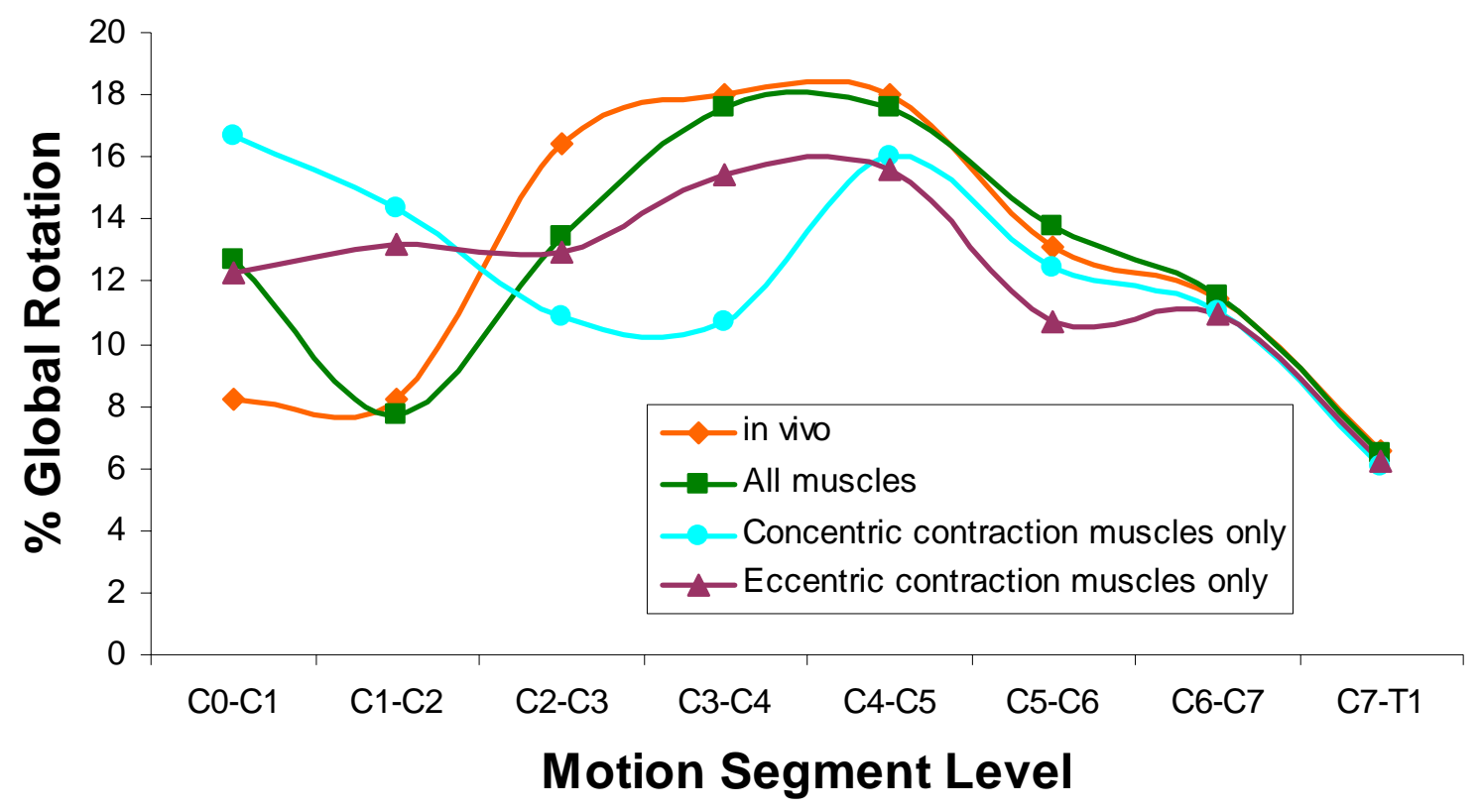

Figure 4-11: Comparisons of the distribution of relative motion segment unit rotations during lateral bending for the "All muscles" mode, "Concentric contraction muscles only" mode, "Eccentric contraction muscles only" mode, and in vivo data. 
data as reported by White and Panjabi, ${ }^{27,41}$ except level C0-C1 which had a higher value. For all three study modes, the rotational contribution to the overall global rotation of levels C4-C5, C5C6, and C7-T1 were comparable to the in vivo values.

\subsubsection{Extension}

In the “All muscles mode”, all the primary muscles involved in both extension and flexion were chosen (Figure 4-12). For the “Concentric contraction muscles only” mode, only the primary muscles involved in extension (extensor muscles) were considered, as shown in Figure 4-13. While in the "Eccentric contraction muscles only" mode, only the primary muscles involved in flexion (forward flexor muscles) were activated (Figure 4-14). Refer to Table 3-7 for the list of extensor and flexor muscles.

The distribution of relative motion segment unit rotation normalized to the global (C0-T1) rotation during extension is shown in Figure 4-15 for different muscle activation modes and in vivo data. Only the simulation results from the "All muscles” mode were comparable to the in vivo data. The results from "Concentric contraction muscles only" mode and "Eccentric contraction muscles only" mode agreed with the in vivo data, except at C3-C4, which was 14\% compared to $11 \%$ for the in vivo case, and at C4-C5, which was $9 \%$ compared to $14 \%$ for the in vivo case. 


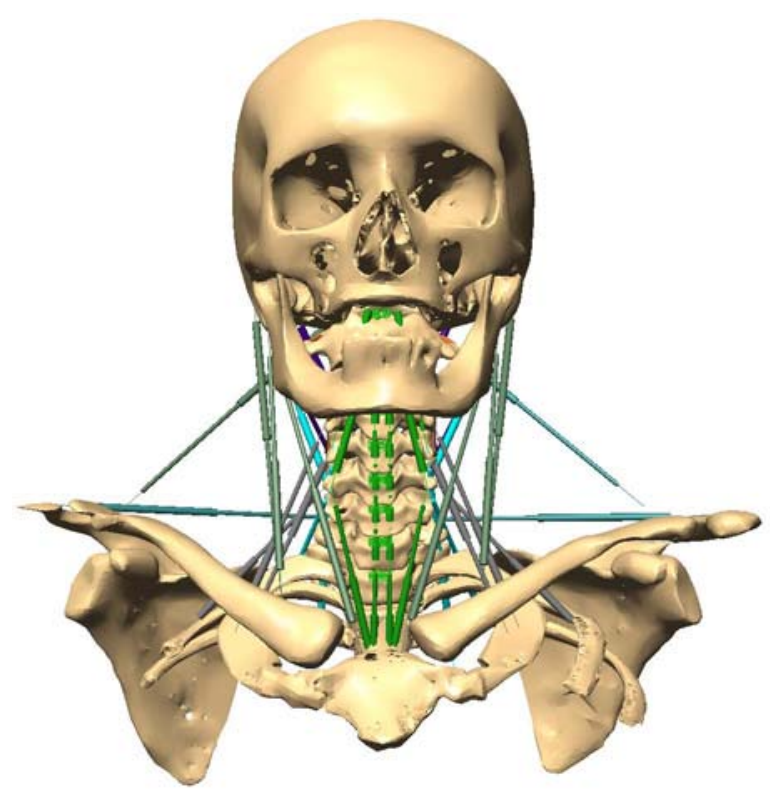

(A)

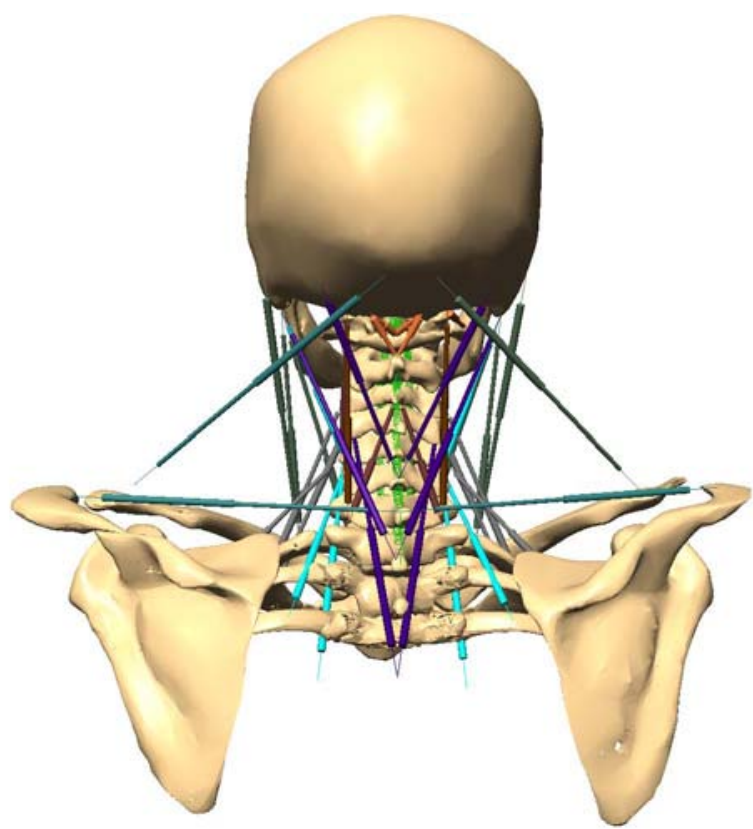

(B)

Figure 4-12: Musculoskeletal head-neck model under “All muscles" mode during extension. (A) Frontal view. (B) Rear view. 


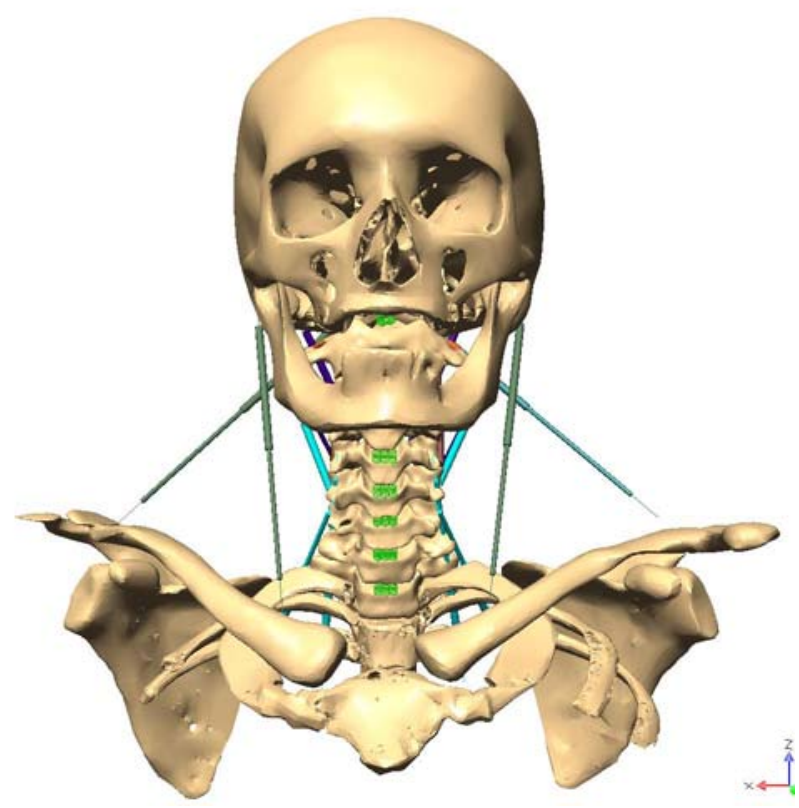

(A)

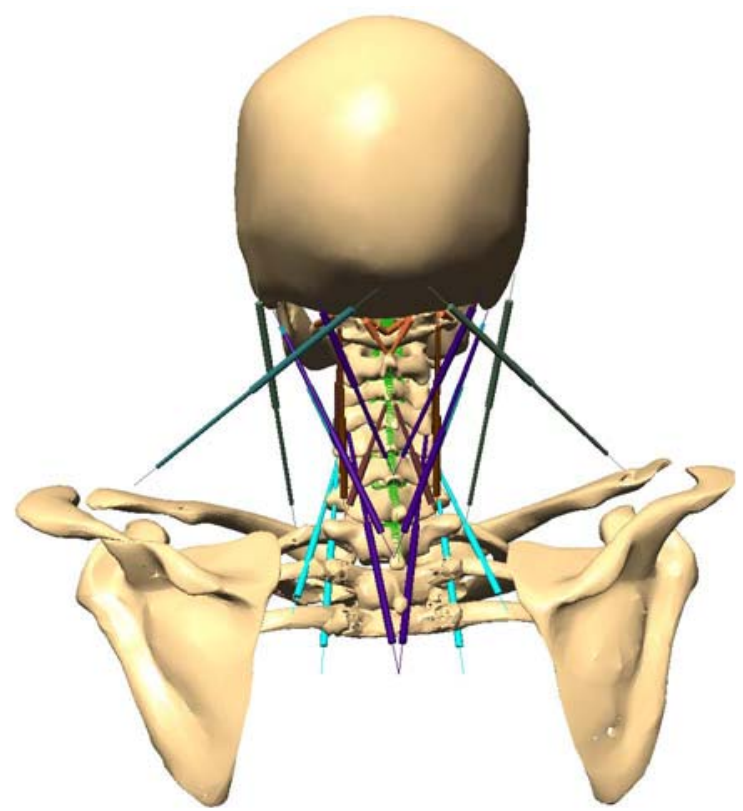

(B)

Figure 4-13: Musculoskeletal head-neck model under "Concentric contraction muscles only” mode during extension. (A) Frontal view. (B) Rear view. 


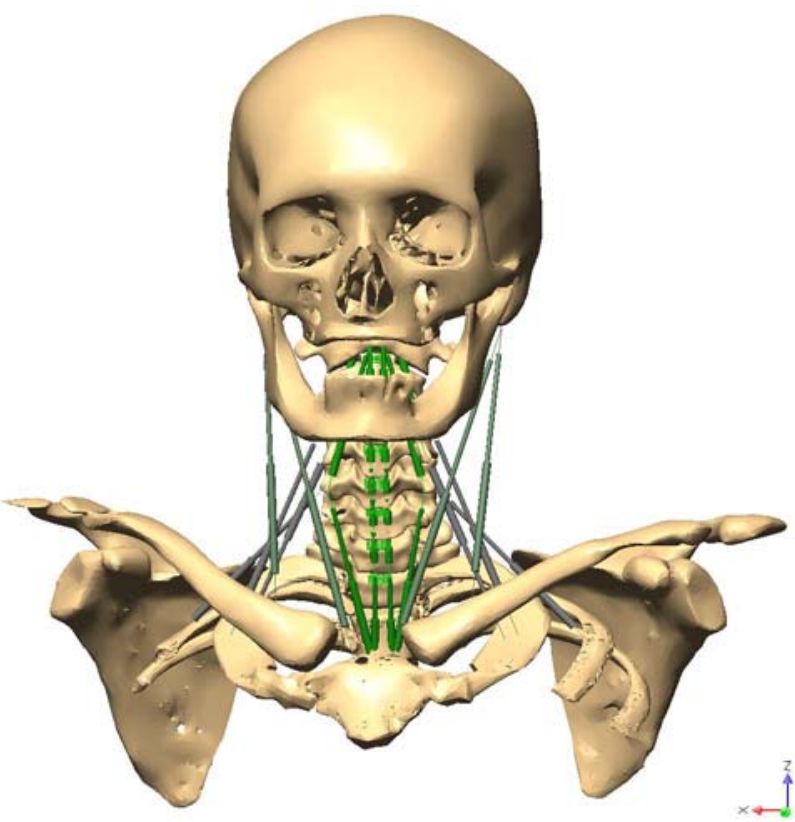

(A)

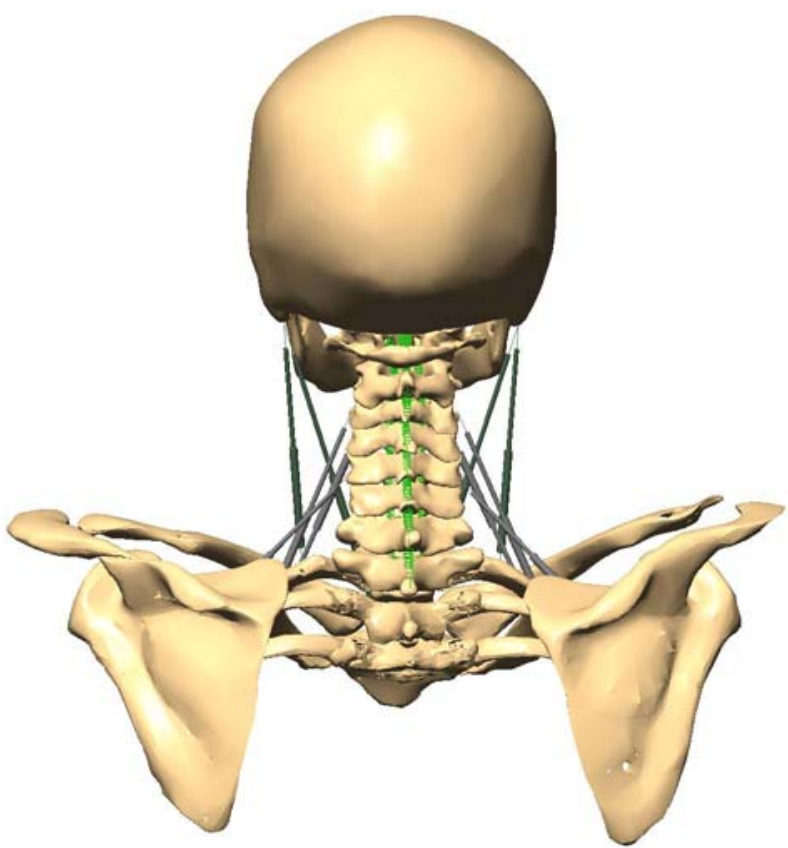

(B)

Figure 4-14: Musculoskeletal head-neck model under "Eccentric contraction muscles only" mode during extension. (A) Frontal view. (B) Rear view. 


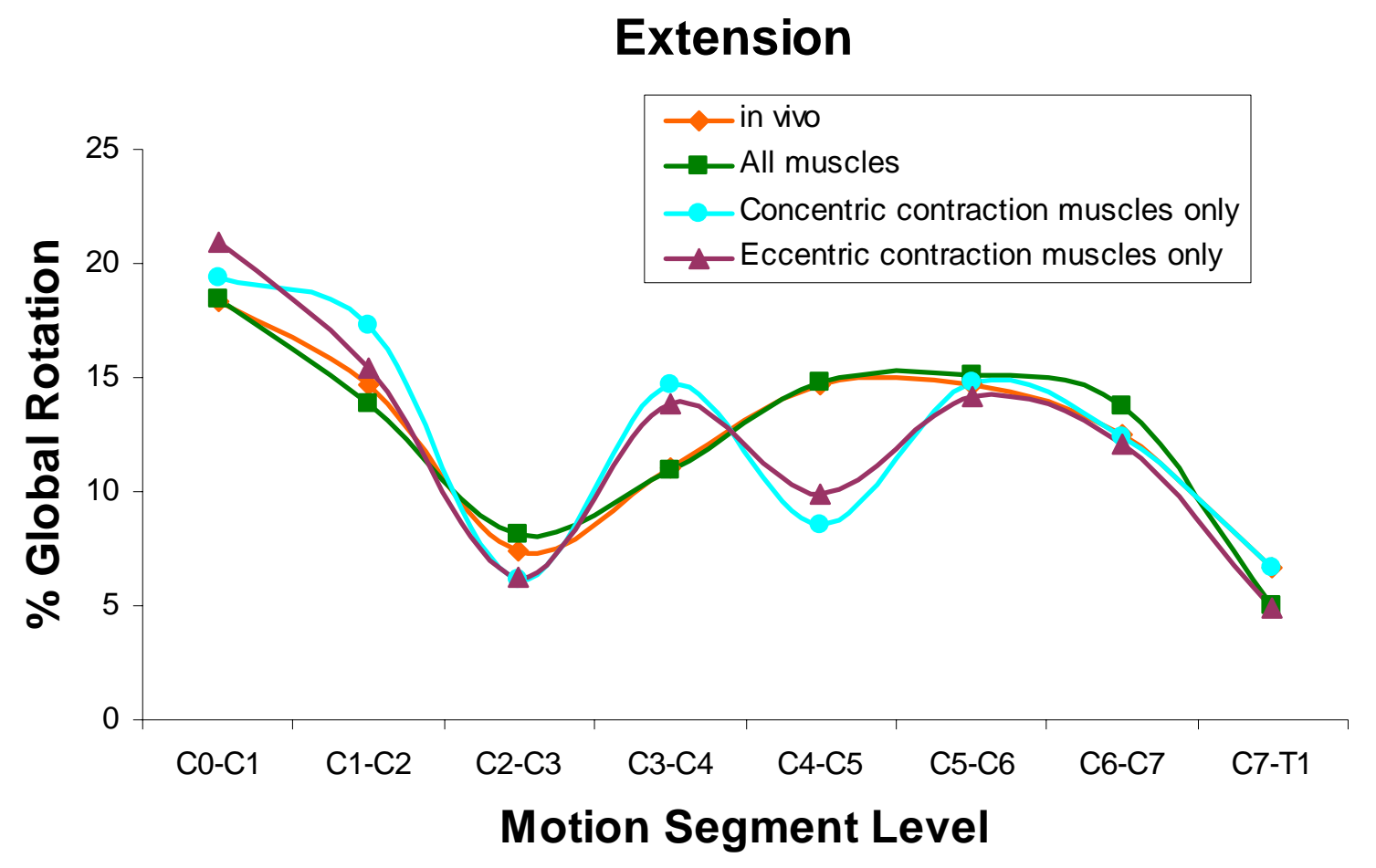

Figure 4-15: Comparisons of the distribution of relative motion segment unit rotations during extension for the "All muscles" mode, "Concentric contraction muscles only" mode, "Eccentric contraction muscles only" mode, and in vivo data. 


\subsubsection{Flexion}

A similar set of muscles used for extension modeling was used to study flexion, as shown in Figure 4-16. The only differences were for the "Concentric contraction muscles only” mode, only forward flexor muscles (i.e. those that shorten) were considered (Figure 4-17), while for “Eccentric contraction muscles only” mode, only those muscles that lengthened (extensor muscles) were activated (Figure 4-18). For the “All muscles” mode, both the primary muscles involved in extension (extensor muscles) and flexion (forward flexor muscles) were chosen. Refer to Table 3-7 for the list of the extensor and forward flexor muscles.

The distribution of relative motion segment unit rotations normalized to the global (C0T1) rotation during extension is shown in Figure 4-19 for different muscle activation modes and in vivo data. All the simulation results were comparable to the in vivo data, except at $\mathrm{C} 0-\mathrm{C} 1$ level, for the "Concentric contraction muscles only", which was 21\% compared to $18 \%$ for the in vivo case. 


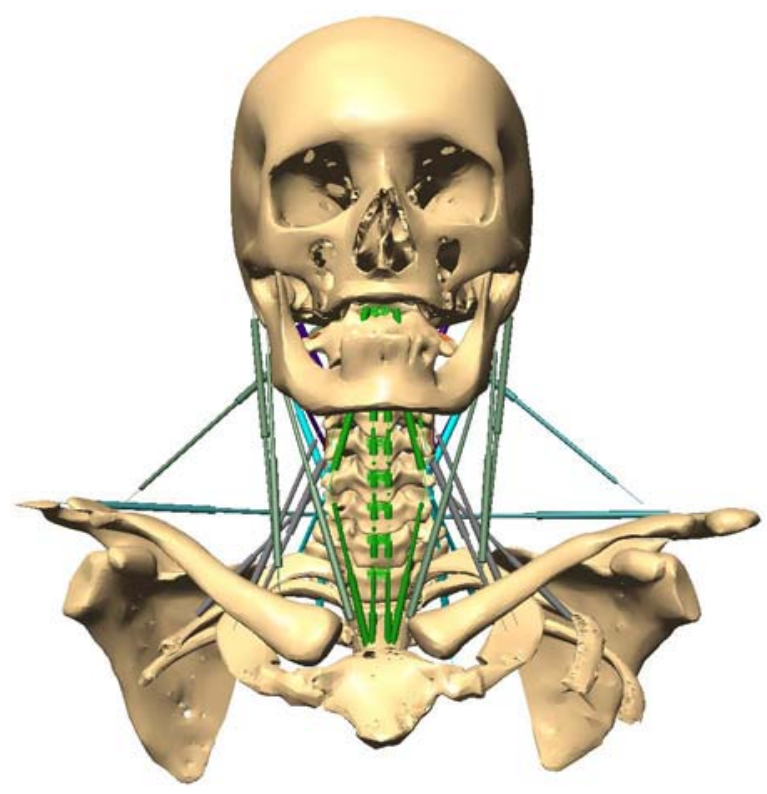

(A)

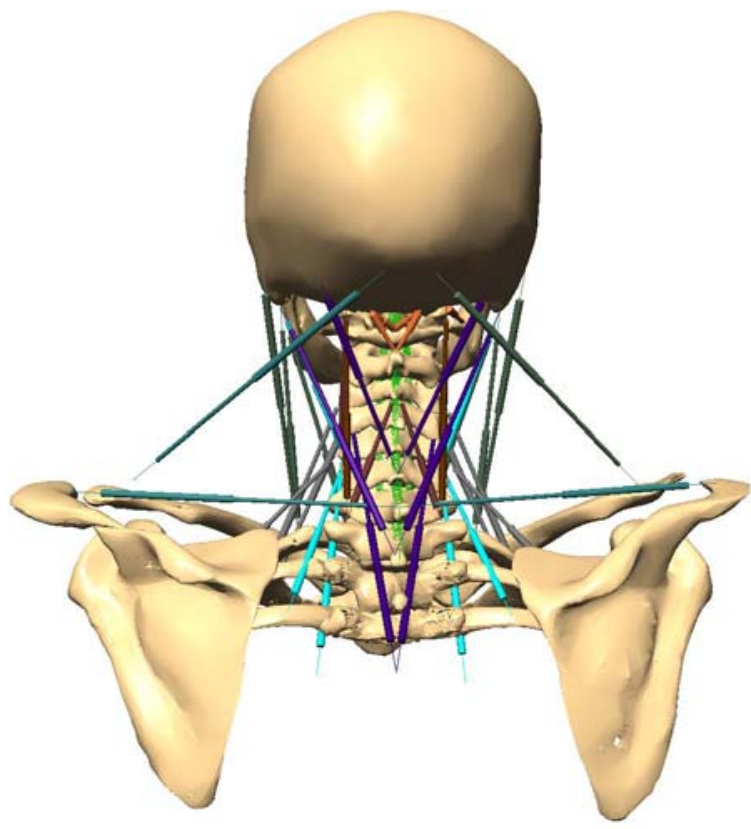

(B)

Figure 4-16: Musculoskeletal head-neck model under “All muscles” mode during flexion. (A) Frontal view. (B) Rear view. 


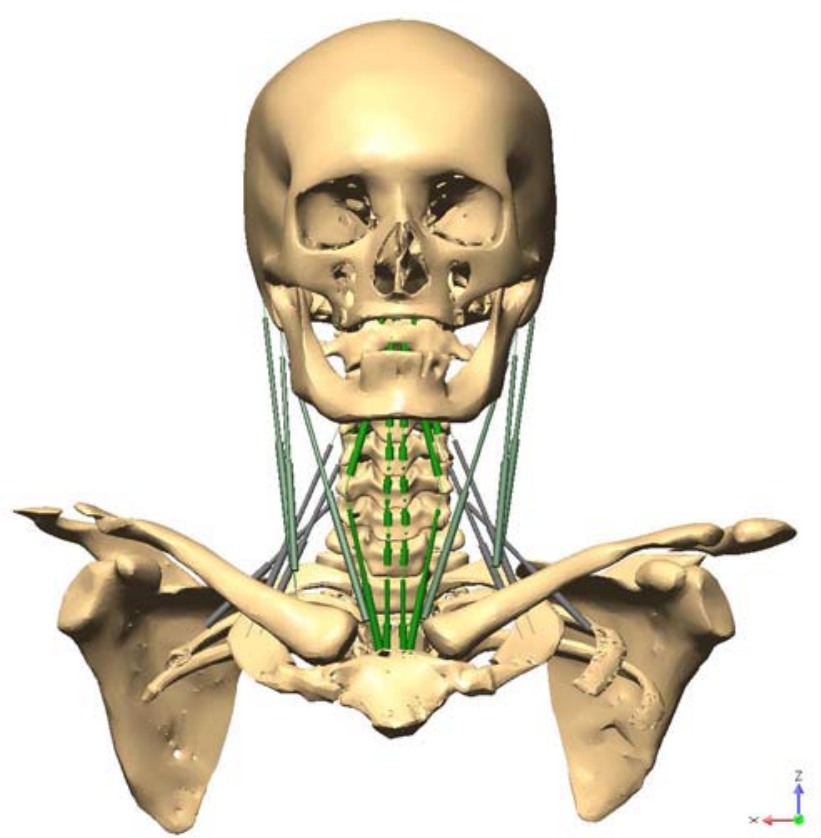

(A)

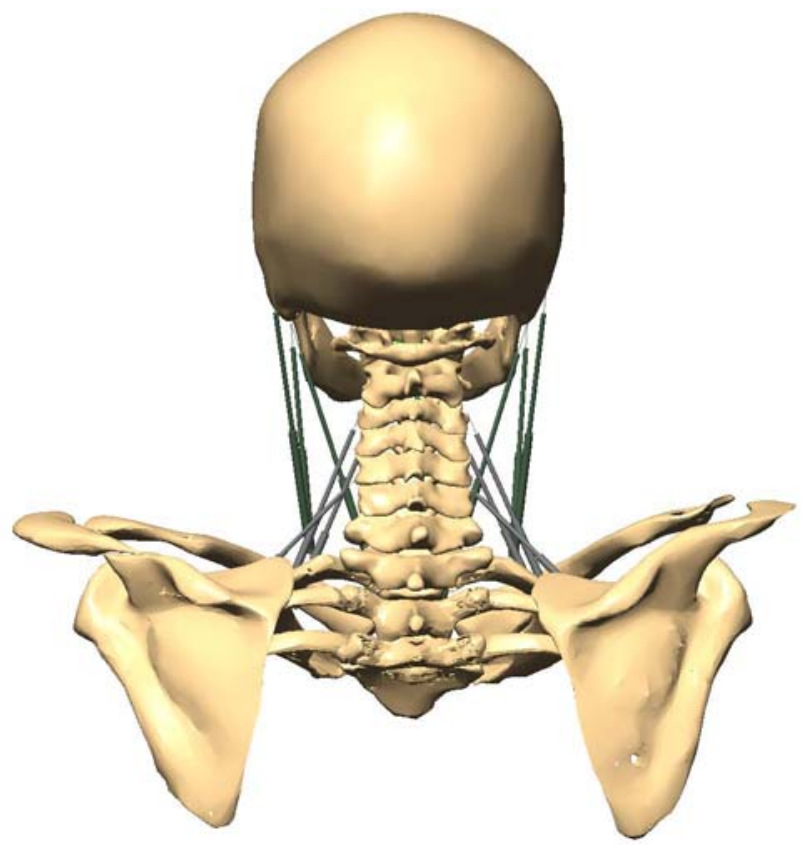

(B)

Figure 4-17: Musculoskeletal head-neck model under "Concentric contraction muscles only” mode during flexion. (A) Frontal view. (B) Rear view. 


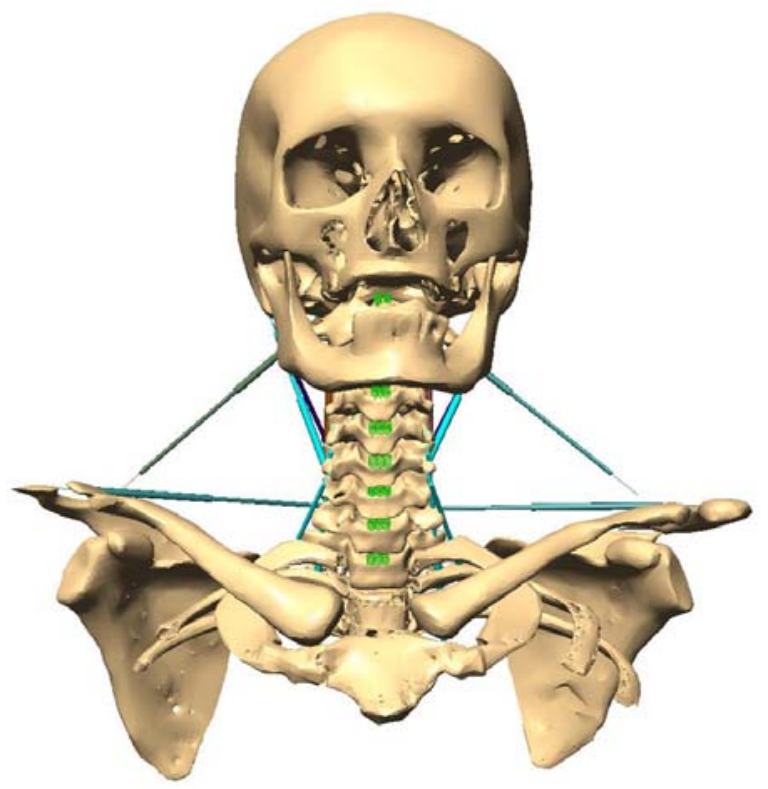

(A)

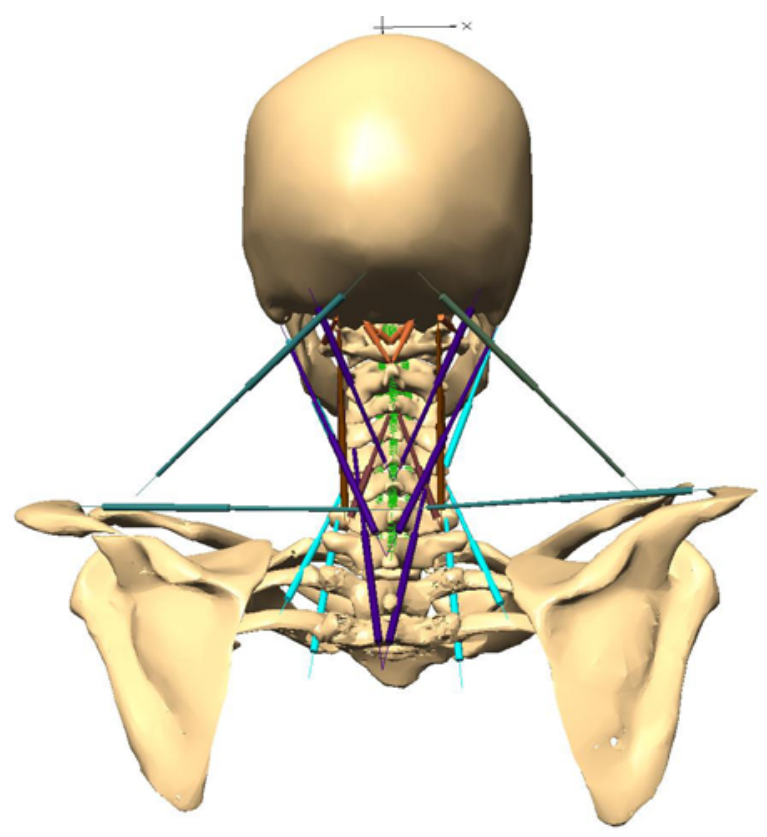

(B)

Figure 4-18: Musculoskeletal head-neck model under "Eccentric contraction muscles only" mode during flexion. (A) Frontal view. (B) Rear view. 


\section{Flexion}

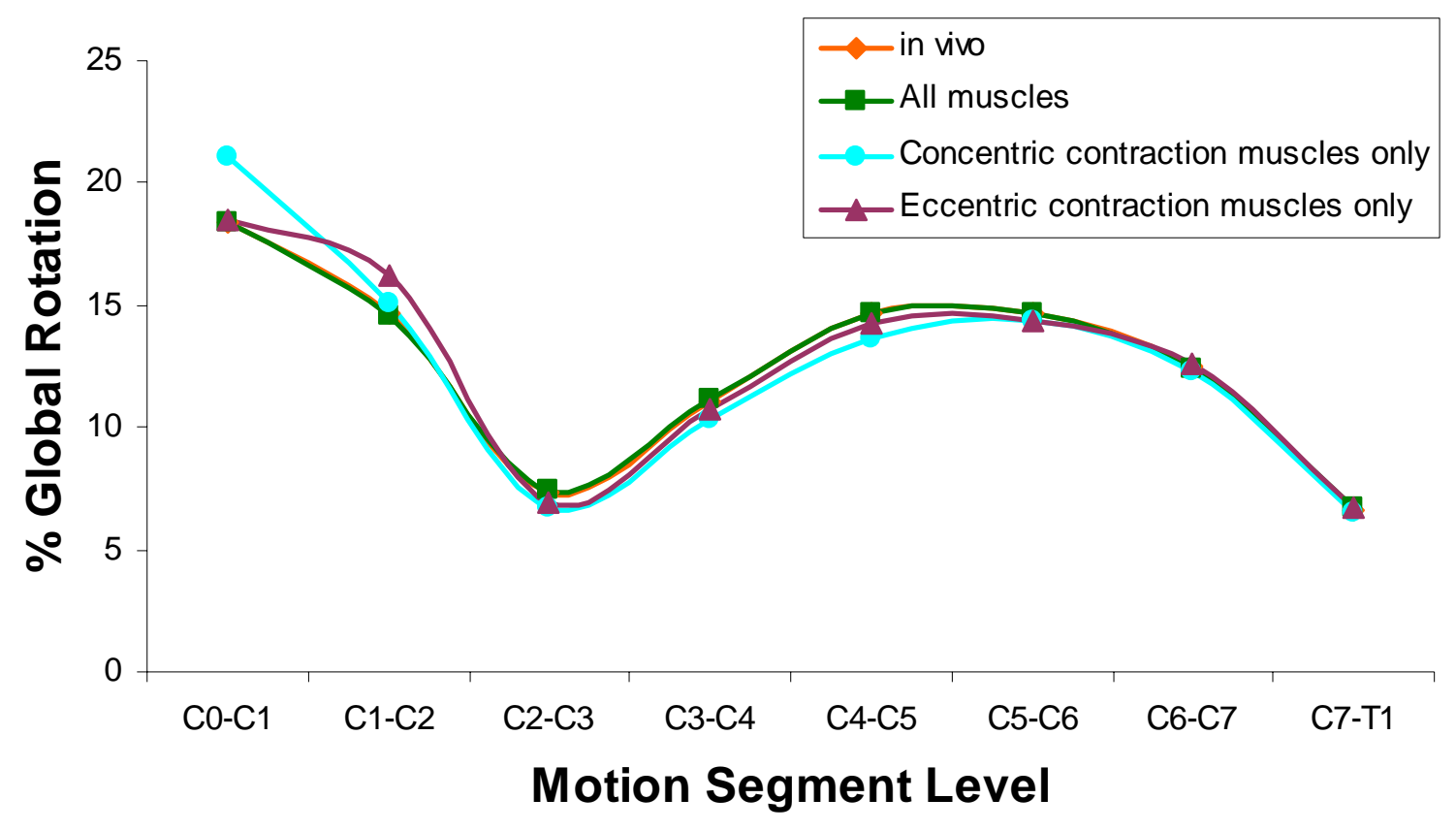

Figure 4-19: Comparisons of the distribution of relative motion segment unit rotations during flexion for the "All muscles" mode, "Concentric contraction muscles only" mode, "Eccentric contraction muscles only" mode, and in vivo data. 


\section{Discussion}

This chapter summarizes the major findings of this study. Section (5.1) discusses the simulation results and some conclusions are drawn. Section (5.2) discusses the limitations with the muscle selection process when developing the model under muscle control, especially for axial rotation and lateral bending. Section (5.3) discusses the similarities and differences between this musculoskeletal model with the previous in vitro model of the sub-axial human

cervical spine developed by Ahn. ${ }^{3}$ Lastly, in order to improve the current model, the limitations of this study are given in Section (5.4).

\subsection{Simulation results}

In Section (4.3), the results of the three study modes for flexion movement matched well with the in vivo data. There are no significant influences of involvement of extensor muscles during flexion. However, the data from the "Eccentric contraction muscles only" mode and the “concentric contraction only” mode for extension neck movement do not agree with the current research outcome. These results imply that the forward flexor muscles play a more important role in extension than the extensor muscles do in flexion. For future work, the contribution of these flexor muscles in extension should be considered as well.

From the simulation results under "All muscles" mode, the translations that occur in the upper cervical spine were very small, which agreed with physiological observations. ${ }^{41}$ 
Furthermore, this finding validated the force-translation data used to define the transtalory stiffness of the C0-C1 and C1-C2 joints.

Coupled motions of axial rotation and lateral bending are very important features in cervical spine kinematics. Clinically, two kinds of coupled motions are especially well established in the cervical spine. ${ }^{27,40}$ Axial rotation is coupled with same direction lateral bending in the lower cervical spine but with opposite direction lateral bending in the upper

cervical spine region. ${ }^{27}$ Lateral bending is accompanied by axial rotation of the same side. ${ }^{27}$ The simulation results obtained from the "All muscles” mode, where left side axial rotation was associated with left-side lateral bending, were in accordance with the previous study by Panjabi. ${ }^{27}$ However, axial rotation was coupled with the same side lateral bending even in the upper cervical region. This finding can be explained by the unavailable definition of the coupling behavior of the upper cervical spine region in the motion-driven model. This study agrees with the results from the current in vitro and in vivo studies to some extent, and provides a reasonable foundation for future modeling work.

\subsection{Comparisons with Ahn model and Vasavada model}

There were several similarities between the current head-neck model and the in vitro model developed by Ahn. ${ }^{3}$ First, the musculoskeletal head-neck model was an extension of the in vitro model. Second, the soft tissue properties in the lower cervical spine region of the model presented in this study were adapted from Ahn. ${ }^{3}$ Lastly, these two models were both developed within the Visual Nastran 4D program. Three major differences existed between these two models. They were the area of focus, model driving forces, and the existence of muscle 
components. Further, the in vitro model developed by $\mathrm{Ahn}^{3}$ only included the sub-axial (C2-T1) spinal segments. A velocity actuator was used to drive the motion response in flexion/extension and lateral bending and a revolute motor was used for axial rotation. The current head-neck models were driven by motion response and muscle length.

Vasavada et al. ${ }^{27,40}$ developed a detailed musculoskeletal head-neck model to investigate how muscle morphometry and moment arms influence moment-generating capacity of human neck muscles in physiologic ranges of motion. The muscle selection of the current head-neck model was based on Vasavada's study. The main difference between Vasavada's model, Ahn’s model, and the current head-neck model, is the modeling platform. Vasavada's model was accomplished in Software for Interactive Musculoskeletal Modeling (SIMM) (Musculographic, Inc. CA), while the other two were developed in Visual Nastran 4D. Table 5-1 shows the main differences between the three models.

\subsection{Muscle selection for axial rotation and lateral bending}

As mentioned in Section (2.1.3), axial rotation and lateral bending are due to unilateral contraction of a number of muscles. Therefore, some muscle contractions will cause the opposite-direction rotation or bending, while some will rotate or bend the head and neck to the same side as their location. Right-side axial rotation and left-side lateral bending were represented in this study. Gray's Anatomy ${ }^{34}$ provided detailed information about which side the neck muscle rotates or bends the head when it is shortening. However, for some muscles, the length-time relationship obtained from the model driven by motion response was in conflict to the description of Gray’s anatomy. ${ }^{2}$ The sternomastoid muscle is an example of when this 
Table 5-1: Comparisons between the current head-neck model, Ahn's model ${ }^{3}$ and Vasavada's model. ${ }^{40}$

\begin{tabular}{c|l|l|l}
\hline $\begin{array}{c}\text { Development } \\
\text { Program }\end{array}$ & Current Model & Vasavada Model & \multicolumn{1}{c}{ Ahn Model } \\
\hline $\begin{array}{c}\text { Interconnecting } \\
\text { Joints }\end{array}$ & $\begin{array}{l}\text { Intervertebral discs, } \\
\text { C0-C1 joint, C1-C2 } \\
\text { joint, Ligaments, } \\
\text { Facet joints }\end{array}$ & SIMM & Visual Nastran 4D \\
\hline Muscles & $\begin{array}{l}\text { 19 distinct neck } \\
\text { muscles }\end{array}$ & $\begin{array}{l}\text { 19 distinct neck } \\
\text { muscles }\end{array}$ & $\begin{array}{l}\text { Intervertebral discs, } \\
\text { Ligaments, Facet } \\
\text { joint }\end{array}$ \\
\hline Area of Focus & $\begin{array}{l}\text { Upper cervical spine } \\
\text { (C0-C2)+Lower } \\
\text { cervical spine (C2- } \\
\text { C7) }\end{array}$ & $\begin{array}{l}\text { Upper cervical spine } \\
\text { (C0-C2)+Lower } \\
\text { cervical spine (C2- } \\
\text { C7) }\end{array}$ & $\begin{array}{l}\text { Lower cervical } \\
\text { spine (C2-C7) }\end{array}$ \\
\hline Driving Force & $\begin{array}{l}\text { 1. Motion response } \\
\text { 2. Muscle length }\end{array}$ & Motion response & $\begin{array}{l}\text { Lateral bending) } \\
\text { 2. Revolute motor } \\
\text { (Axial rotation) }\end{array}$ \\
\hline
\end{tabular}


occurred. According to Gray's anatomy, ${ }^{2}$ the left-side sternomastoid is supposed to shorten and rotate the cervical spine to the right side in right-side axial rotation. However, based on the results, it was the right-side sternomastoid muscle that shortened during right-side axial rotation. This can be explained by the significant coupled motion of axial rotation and lateral bending which occurs in the cervical spine. A shortening sternomastoid muscle also contributes to the same side lateral bending. Therefore, in the coupled right-side axial rotation and right-side lateral bending, the right-side sternomastoid muscle shortens. The response of the motion-driven model confirmed this explanation. It was found that the left-side sternomastoid contracted in right-side axial rotation, which was in a very close agreement with the description of the anatomical reference. This explanation can also be applied to some muscles that are not the major function muscles in lateral bending and rotate the spine to the opposite side when contracting. For instance, the left side of longus colli-inferior oblique and scalenus anterior muscles, were activated in right-side axial rotation in the model driven by muscle length, not the right-side ones as the anatomy reference suggested. A similar explanation can be applied. The right side of the muscles longus colli-inferior oblique, longus colli-superior oblique, and scalenus anterior were supposed to shorten during left-side lateral bending since they rotate the neck to the right side, however, a descending trend was found in the length-time curves of the left-side muscles. 


\subsection{Limitations}

The limitations of this study were divided into two parts: (1) the limitations of defining the soft tissue structures within the anatomical model development, and (2) the limitations of the methods used in this study, including the inverse dynamics and the method for model evaluation.

\subsubsection{Anatomical model development}

No simulation model is without limitations. The model developed in this study is not an exception. This model shares some common limitations with the one developed by Ahn. ${ }^{3}$ This model does not have a general representation due to the singularity of the vertebral body geometries, and the initial alignment of the cervical column may not reflect neutral alignment of the general population. $\mathrm{Ahn}^{3}$ provided a detailed description of process used to model the soft tissue structures of the lower cervical spine.

In this study C0-C1-C2 complex was modeled as simple spherical joints at C0-C1 and C1-C2. In addition, four types of ligaments (AA-OM, PA-OM, ALL and LF) were identified. However, since the moving axis of rotation of the spinal bodies changes during the movement, ${ }^{41}$ the physiologic behavior of the complex structure of C0-C1-C2 could not be completely simulated. In addition, the translatory stiffness properties for the upper cervical spine joints were derived from the ones used in the discs of the lower cervical spine due the unavailability of published data. 
Muscles were represented by straight-line elements in Visual Nastran 4D, which was a limiting factor because the simulated muscles could not exactly replicate the intact muscle structure. For example, the muscle cleidomastoid was modeled as a very long actuator inserting between clavicle and skull (Figure 5-1). In addition, if a muscle inserts over a large area of bone, it cannot have multiple attachment sites. In this model, some muscle was separated into two or more portions. ${ }^{23}$ For instance, the muscle longus colli-vertical attaching from C1 to T3 (the third thoracic body) was model as seven separate actuators that inserted between each vertebra body (Figure 5-2). However, for future studies muscles should have the ability to follow the curvature of the neck, resulting in more realistic muscle force line of action. ${ }^{39}$

\subsubsection{Inverse dynamics}

The inverse dynamics method used in this study is not the same as the traditional approach cited in the biomechanical literature. ${ }^{33}$ The definition of inverse dynamics is a process of deriving the kinetics response for a given motion from the kinematics of the motion itself. However, Visual Nastran 4D was not designed to model muscles and lacked the ability to calculate the muscle forces through muscle properties, such as PCSA (physiologic crosssectional area), distribution of muscle fiber type, or muscle velocity-length properties. Thus, the only information obtained from the kinematics model (model driven by motion response) was muscle length. However, neck movement is caused by the muscle contraction that generates muscle forces. As a result, the muscle-driven model was actually driven under muscle length control, and could not simulate the in vivo human neck movement. 


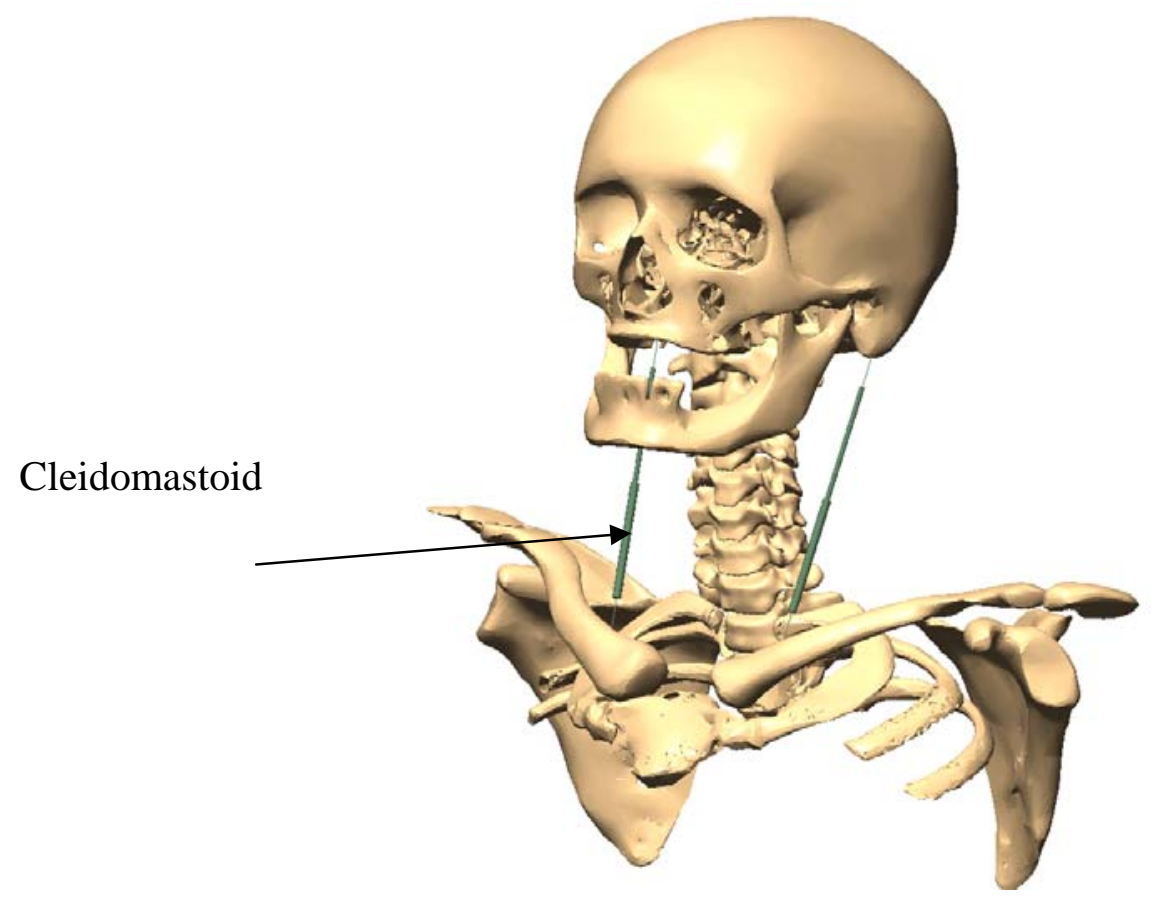

Figure 5-1: The muscle cleidomastoid.

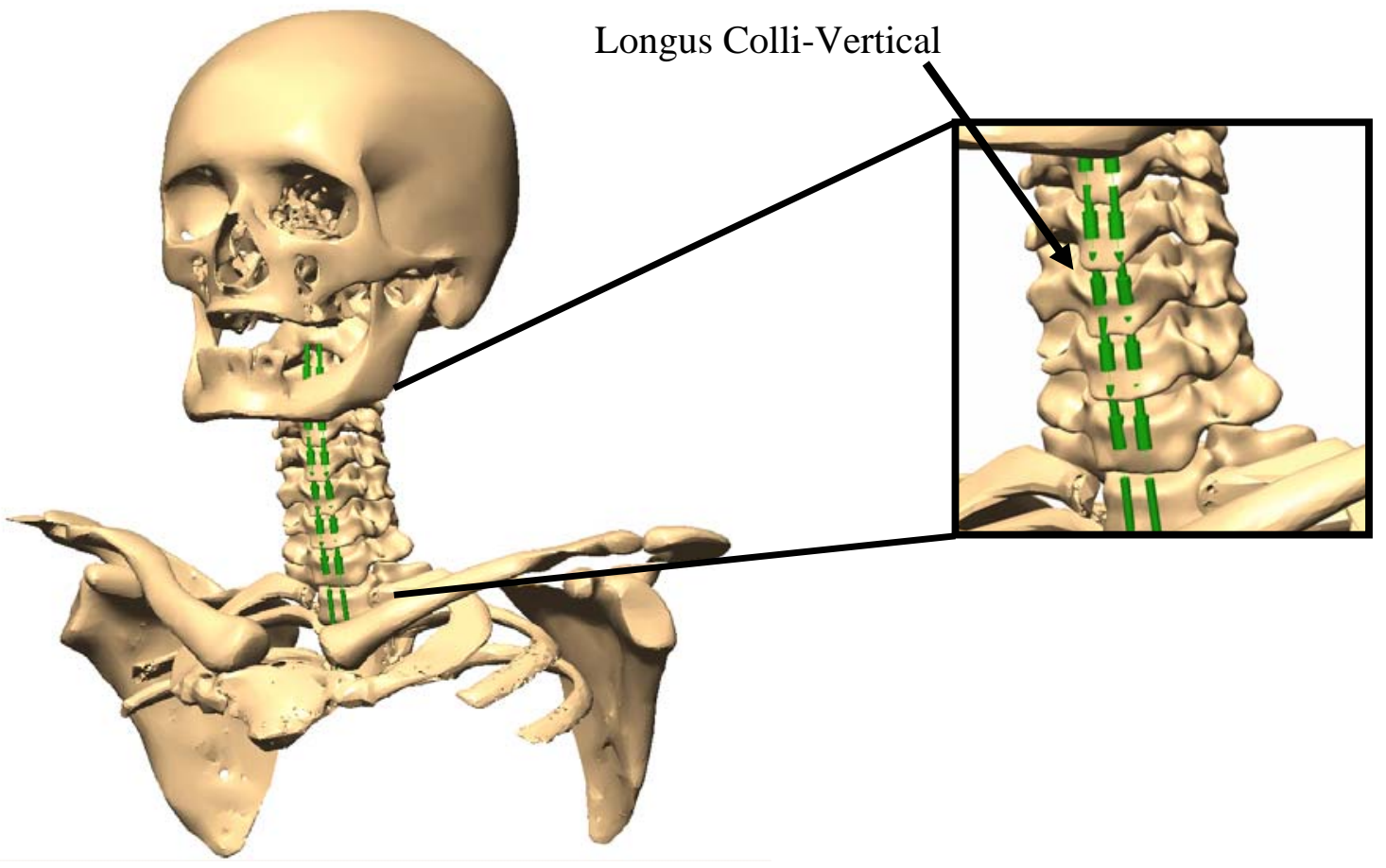

Figure 5-2: The muscle longus colli-vertical. 
Inverse dynamics approach requires an accurate description of the motion response to obtain physiological results in biomechanical studies. However, the input data for the motion driven model discussed in Section (3.2.2) were from two sources: the output data from Ahn's in vitro model for the lower cervical spine region and in vivo data from literature for the upper cervical spine region. Ahn's in vitro model focused on obtaining a close match with the rotational contribution to the global rotation, but it did not include any analysis of the translational parameters. In addition, the coupled motions were not considered in the upper cervical spine. These two factors limited the accuracy of the results when using the inverse dynamics method and the same limitations noted with the response of motion segment unit rotations associate with the in vitro model are also present in this muscle model. However, by using the inverse dynamics method, the information of the muscle length-time relationship needed to drive the musculoskeletal head-neck model was available. Future work should introduce a forward dynamics simulation, which is a powerful approach for investigating how the elements of the musculoskeletal system interact to produce movement. ${ }^{38}$

\subsubsection{Methods of model evaluation}

Evaluation of the kinematics response only compared the motion segment unit rotations to the in vivo published data reported by White and Panjabi. ${ }^{41}$ A detailed analysis should include the coupling characteristics that occur between lateral bending and axial rotation. Another parameter that should be added to the kinematic analysis is the three-dimensional helical axis of motion (HAM) between adjacent vertebrae. 


\section{Conclusion and future work}

This chapter includes two parts. A general conclusion of this study is given in Section (6.1). Section (6.2) suggests future work, including some recommendations for improving the anatomical model and the application of a muscle control program, such as the "Virtual Muscle" program.

\subsection{Concluding discussion}

A computational model of the human head-neck musculoskeletal system was developed that simulated the dynamic motion response of the head and cervical spine under physiologic head movements. This model included 19 distinct major muscles of the neck. According to the motor function of the muscles, the muscles were categorized to form the primary muscle group for each motion. The motion-driven model provided excellent replication of in vivo vertebral kinematics. A similar response occurred for the muscle-driven model when the muscle groups on both sides were activated. Although there was no significant involvement of the extensor muscles during flexion, the forward flexor muscle played an important role during extensional

head movement. In the future, this model can be used to explore muscle control strategies within the "Virtual Muscle" program to simulate EMG muscle force activation conditions. 


\subsection{Future work}

A computational approach to model the complex mechanical properties of muscles and tendons under physiological conditions is introduced. ${ }^{10}$ Furthermore, recommendations for improving the anatomical model are given in this section.

\subsubsection{The application of "Virtual Muscle" program}

This study presented the development of a musculoskeletal head-neck model that simulated four different movements under muscle length control. The next developmental step is to add the muscle force capabilities to simulate the in vivo active muscle motion response. There are two methods to determine the muscle force in the simulation model. One method is formulate a static optimization solution that solves different optimization problems at each instant during the movement to calculate the muscle force. ${ }^{23}$ In this method, the net joint torques are solved from the same kinematic data used in the inverse dynamics solution. The distribution of activations among muscles that could contribute to such net torques is solved by minimizing some criterion such as the total torque or total energy consumption. This could be extremely difficult to do in a structure like the neck, which is dominated by viscoelastic properties rather than Newtonian mechanics (personal communication with Dr. Gerald Loeb from University of South Californica and Jun Yan, 2004). The second method is to use EMG data to calculate the corresponding muscle forces. $^{20,21}$ 
The integration of muscle control strategies through the use of the "Virtual Muscle" program may be a possible solution to the second method. The Visual Nastran 4D program allows easy integration of many programs, such as Matlab (The MathWorks, Natick, MA) and Pro/ENGINEER (Parametric Technology Corporation, Needham, MA). The "Virtual Muscle" program is embedded within Matlab/Simulink and it allows the user to assign different muscle fiber types that are based on the muscle morphometry data, such as the physiological crosssectional area of the muscles and the fascicle length. ${ }^{9,10}$ With the architectural parameters of the muscles defined in the "Virtual Muscle" program, a simulation block that calculates the muscle force production is built. The simulation muscle block would require two inputs as shown in Figure 6-1: the musculotendon path length in units of centimeter and the neural activation level (between 0 and 1). An EMG signal that represents the neural activity can be scaled to the level of maximal voluntary contraction and used to control the muscle response. ${ }^{9}$ In order to integrate the "Virtual Muscle" program in Visual Nastran 4D, only the force actuator component would need to be modified.

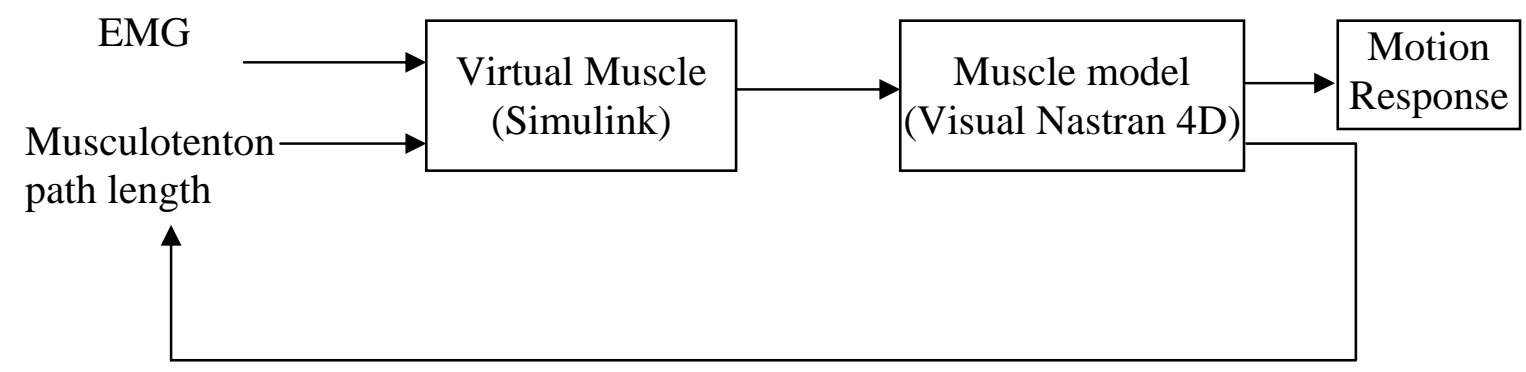

Figure 6-1: Interactions between "Virtual Muscle” and Visual Nastran 4D. 
A forward dynamic simulation can be performed by integrating the model with a set of Simulink blocks that describe the properties of the musculoskeletal system. In contrast to the inverse dynamics method, the forward dynamics method uses muscle excitations as inputs to calculate the corresponding body motions. ${ }^{13,24,30,31,43,47}$ EMG data would drive the muscle mechanics in Simulink, which in turn would drive the skeletal model in Visual Nastran 4D. ${ }^{10}$ Further, the muscle mechanics are modeled in the "Virtual Muscle" program computationally and the existing feedback would enhance the stability of the model. The interactions between these programs are shown in Figure 6-1.

In order to generate the muscle block, information of the muscle fiber type and muscle morphometry are needed to be properly defined in the "Virtual Muscle" program. However, not all the properties for the muscles used in this musculoskeletal head-neck model are available, due to the lack of research in this field.

The morphologic and functional characteristics of the neck muscles are not well defined due to the complicated nature of the human neck musculature. ${ }^{18}$ Kamibayashi et al. ${ }^{18}$ created a systematic database of morphometric parameters for human neck muscles. As such, some of the data needed to model the neck muscles in the "Virtual Muscle" program, such as PCSA and mass of the muscle, can be directly derived from their study. Alternatively, some muscle parameters not directly available in the literature can be calculated using other unknown muscle property values and their relationships. For example, the "Virtual Muscle" program requires that the tendon length be input but it is unavailable in the published literatures. Zajac ${ }^{46}$ has established the relationship between the tendon slack lengths and the length of the tendon at the muscle's optimal force. This approach offers an alternative way to calculate the tendon length by using slack tendon length values provided in literatures. ${ }^{18,40}$ 
As for the muscle fiber types, the comparison of the vertebral muscle fiber characteristics between human and rhesus monkey shows there are considerable similarities between the deep muscle samples of these two species. ${ }^{4}$ This finding indicates that some research on the muscle fiber of rhesus monkey may be used to define the human muscles’ properties. Richmond et al. ${ }^{32}$ described the distribution of each fiber type in the rhesus monkey neck muscles in detail. This information could be used to model human neck muscles in "Virtual Muscle" program.

\subsubsection{Recommendation for improving the anatomical model}

To enhance the development of the joints of the upper cervical spine in this model, the C0-C1-C2 complex can be connected by nonlinear viscoelastic ligaments that include an anterior membrane, posterior membrane, capsular ligament, alar ligament, transverse ligaments, and the

nonlinear viscoelastic facet joints. ${ }^{14}$ The technique used to make the facet joints in the sub-axial spine would not work for the C0-C1-C2 complex, since the lower facets of C1 and the upper facets of C2 are convex, whereas the facet joints of the sub-axial spine (C2-T1) have flat articular surfaces. This anatomy allows C1 to descend and ascend relative to C2 during axial rotation. ${ }^{5}$ Bioconvex surfaces that can collide with each other need to be developed to simulate this movement. In addition, some amount of overlap should be allowed for enhancing the model accuracy. $^{3}$

Muscles were modeled in Visual Nastran 4D with actuator, which functioned as straightline components. In future studies, the muscles should be allowed to follow the curvature of the spine. One approach to do this is to connect multiple actuators together along the curved path. ${ }^{3}$ However, this would result in an increase in computational time, different shortening rate, and 
the need for multiple actuator components per muscle. Having multiple actuators per muscle groups would also not be easy to adapt to the "Virtual Muscle" program. Further, a more sophisticated control strategy is needed to balance all the extra components. Another approach is to choose a commercially-available musculoskeletal modeling program, such as SIMM. SIMM is a graphics-based software package that enables the users to quickly develop and analyze musculoskeletal models. In SIMM, muscle-tendon actuators can be defined as spheres, ellipsoids, and cylinders to wrap over the bones. However, there are some limitations with SIMM. SIMM does not integrate with Simulink as easily as Visual Nastran 4D. In order to calculate the muscle force in the "Virtual Muscle" program, a second program would be needed to allow the "Virtual Muscle” program to interact with SIMM. The Muscuoskeletal Modeling in Simulink (MMS) is a software developed by A.E. Mann Institute for Biomedical Engineering at University of South California for building computer models of the musculoskeletal systems in Matlab’s Simulink environment. It may be possible to use this to serve as the bridge between SIMM and the "Virtual Muscle” program. Another limitation with SIMM is that each joint can only be modeled with three rotational degrees of freedom; no translation motion is permitted.

\subsubsection{Applications of this model}

There are many applications for this musculoskeletal head-neck model. First, it could be used as a design tool developing and improving instrumentation. Second, this model can be used to study the effect of surgical techniques on spinal kinematics and kinetics compared to a normative group. Third, it could provide an in vivo simulation environment for the investigation of ligamentous, intervertebral disc, or muscular injuries, as the soft tissue injuries are a major 
reason for the neck injuries. Last,ly, it could readily support research on each injury caused by car collisions (e.g. Whiplash Associated Disorders), which cannot be accomplished in vivo. 14,17,39 


\section{List of references}




\section{List of reference}

1. Ackerman MJ, Banvard RA. Imaging outcomes from the National Library of Medicine's Visible Human Project. Comput Med Imaging Graph 2000;24:125-6.

2. Agur A, Lee, MJ, ed. Grant's Atlas of Anatomy. 9th ED. ed. Baltimore: Williams \& Wilkins, 1991.

3. Ahn HS. A Virtual Model of the Human Cervical Spine for Physics-based Simulation and Applications. PhD. Thesis. Department of Biomedical Engineering. Memphis, Tennessee: The University of Tennessee Health Science Center, 2005.

4. Bagnall KM, Ford DM, McFadden KD, et al. A comparison of vertebral muscle fiber characteristics between human and monkey tissue. Acta Anat (Basel) 1983;117:51-7.

5. Bogduk N, Mercer S. Biomechanics of the cervical spine. I: Normal kinematics. Clin Biomech (Bristol, Avon) 2000;15:633-48.

6. Bridwell K. Muscles of The Spine. Website:http://spineuniverse.com/displayarticale.php/article1272.html Access on Feb 272006.

7. Brolin K, Halldin P, Leijonhufvud I. The effect of muscle activation on neck response. Traffic Inj Prev 2005;6:67-76.

8. Chao EY. Graphic-based musculoskeletal model for biomechanical analyses and animation. Med Eng Phys 2003;25:201-12.

9. Cheng EJ, Brown IE, Loeb GE. User's Manual: Virtual Muscle 3.1.5 Muscle Model for Matlabed, 2001.

10. Cheng EJ, Brown IE, Loeb GE. Virtual muscle: a computational approach to understanding the effects of muscle properties on motor control. J Neurosci Methods 2000;101:117-30.

11. Clark CR. The Cervical Spine: The cervical spine research society. 3rd ed. Philadelphia: Lippincott- Raven Publishers, 1998.

12. Cripton PA, Sati M, Orr TE, et al. Animation of in vitro biomechanical tests. J Biomech 2001;34:1091-6.

13. Davy DT, Audu ML. A dynamic optimization technique for predicting muscle forces in the swing phase of gait. J Biomech 1987;20:187-201.

14. De Jager MKJ. Mathematical Head-Neck Model for Accleration Impacts. PhD. Thesis: Eindhoven University of Technology, 1996. 
15. Deng YC, Goldsmith W. Response of a human head/neck/upper-torso replica to dynamic loading--II. Analytical/numerical model. J Biomech 1987;20:487-97.

16. Fagan MJ, Julian S, Mohsen AM. Finite element analysis in spine research. Proc Inst Mech Eng [H] 2002;216:281-98.

17. Garcia T, Ravani B. A biomechanical evaluation of whiplash using a multi-body dynamic model. J Biomech Eng 2003;125:254-65.

18. Kamibayashi LK, Richmond FJ. Morphometry of human neck muscles. Spine 1998;23:1314-23.

19. Krabbel G, Appel H. Development of a finite element model of the human skull. J Neurotrauma 1995;12:735-42.

20. Lloyd DG, Besier TF. An EMG-driven musculoskeletal model to estimate muscle forces and knee joint moments in vivo. J Biomech 2003;36:765-76.

21. Manal K, Gonzalez RV, Lloyd DG, et al. A real-time EMG-driven virtual arm. Comput Biol Med 2002;32:25-36.

22. Milne N. The role of zygapophysial joint orientation and uncinate processes in controlling motion in the cervical spine. J Anat 1991;178:189-201.

23. Pandy MG. Computer modeling and simulation of human movement. Annu Rev Biomed Eng 2001;3:245-73.

24. Pandy MG, Zajac FE, Sim E, et al. An optimal control model for maximum-height human jumping. J Biomech 1990;23:1185-98.

25. Panjabi MM. Cervical spine models for biomechanical research. Spine 1998;23:2684-700.

26. Panjabi MM, Crisco JJ, 3rd, Lydon C, et al. The mechanical properties of human alar and transverse ligaments at slow and fast extension rates. Clin Biomech (Bristol, Avon) 1998;13:11220.

27. Panjabi MM, Crisco JJ, Vasavada A, et al. Mechanical properties of the human cervical spine as shown by three-dimensional load-displacement curves. Spine 2001;26:2692-700.

28. Panjabi MM, Oxland TR, Parks EH. Quantitative anatomy of cervical spine ligaments. Part II. Middle and lower cervical spine. J Spinal Disord 1991;4:277-85.

29. Pedrocchi A, Pedotti A, Baroni G, et al. Inverse dynamic investigation of voluntary trunk movements in weightlessness: a new microgravity-specific strategy. J Biomech 2003;36:1691700 . 
30. Piazza SJ, Delp SL. The influence of muscles on knee flexion during the swing phase of gait. J Biomech 1996;29:723-33.

31. Raasch CC, Zajac FE, Ma B, et al. Muscle coordination of maximum-speed pedaling. J Biomech 1997;30:595-602.

32. Richmond FJ, Singh K, Corneil BD. Neck muscles in the rhesus monkey. I. Muscle morphometry and histochemistry. J Neurophysiol 2001;86:1717-28.

33. Riener R, Straube A. Inverse dynamics as a tool for motion analysis: arm tracking movements in cerebellar patients. J Neurosci Methods 1997;72:87-96.

34. Salmons S. Muscle. In Bannister HL, Berry, M. Martin, ed. Gray's Anatomy. 38th ed. New York: Churchill Livingstone Inc., 1995.

35. Sances A, Jr., Myklebust JB, Maiman DJ, et al. The biomechanics of spinal injuries. Crit Rev Biomed Eng 1984;11:1-76.

36. Shea M, Edwards WT, White AA, et al. Variations of stiffness and strength along the human cervical spine. J Biomech 1991;24:95-107.

37. Stemper BD, Kumaresan S, Yoganandan N, et al. Head-neck finite element model for motor vehicle inertial impact: material sensitivity analysis. Biomed Sci Instrum 2000;36:331-5.

38. Thelen DG, Anderson FC, Delp SL. Generating dynamic simulations of movement using computed muscle control. J Biomech 2003;36:321-8.

39. van der Horst MJ. Human Head Neck Response in Frontal, Lateral and Real End Impact Loading: modelling and validation. PhD. Thesis: Technical University of Eindhoven, 2002.

40. Vasavada AN, Li S, Delp SL. Influence of muscle morphometry and moment arms on the moment-generating capacity of human neck muscles. Spine 1998;23:412-22.

41. White AA, Panjabi MM. Clinical Biomechanics of the Spine. 2nd ed. Philadelphia: J.B. Lippincott Company, 1990.

42. Williams JL, Belytschko TB. A three-dimensional model of the human cervical spine for impact simulation. J Biomech Eng 1983;105:321-31.

43. Yamaguchi GT, Zajac FE. Restoring unassisted natural gait to paraplegics via functional neuromuscular stimulation: a computer simulation study. IEEE Trans Biomed Eng 1990;37:886902.

44. Yoganandan N, Kumaresan S, Pintar FA. Biomechanics of the cervical spine Part 2. Cervical spine soft tissue responses and biomechanical modeling. Clin Biomech (Bristol, Avon) 2001;16:1-27. 
45. Yoganandan N, Pintar FA, Sances A, Jr., et al. Strength and motion analysis of the human head-neck complex. J Spinal Disord 1991;4:73-85.

46. Zajac FE. Muscle and tendon: properties, models, scaling, and application to biomechanics and motor control. Crit Rev Biomed Eng 1989;17:359-411.

47. Zajac FE. Muscle coordination of movement: a perspective. J Biomech 1993;26 Suppl 1:109-24.

48. Zong Z, Lee HP, Lu C. A three-dimensional human head finite element model and power flow in a human head subject to impact loading. J Biomech 2006;39:284-92. 


\section{Appendices}


Appendix A Detailed muscle model 
Longus capitis and colli

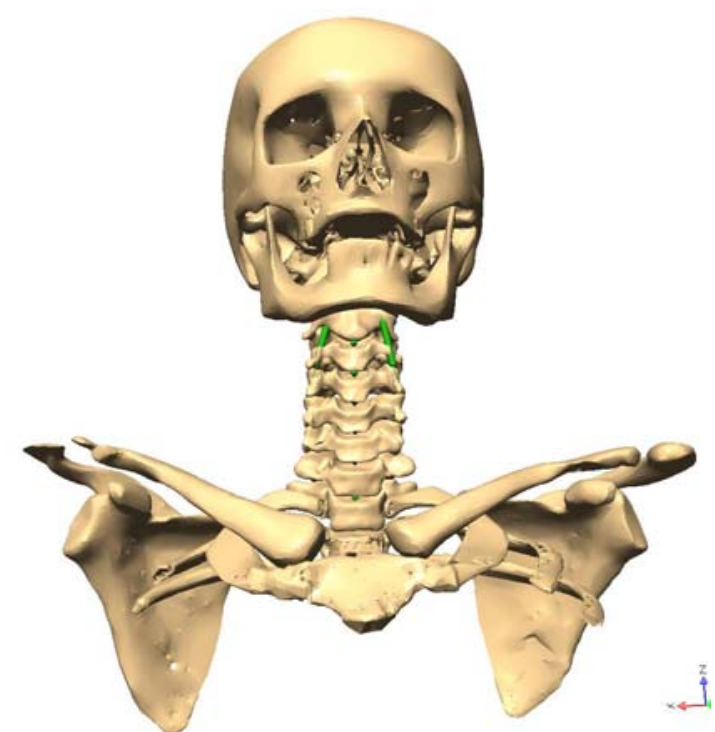

(A)

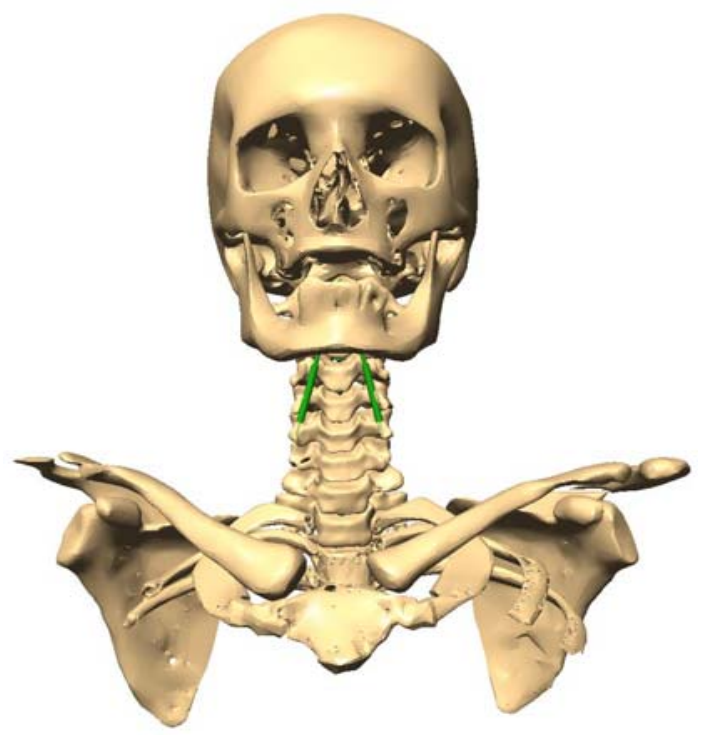

(C)

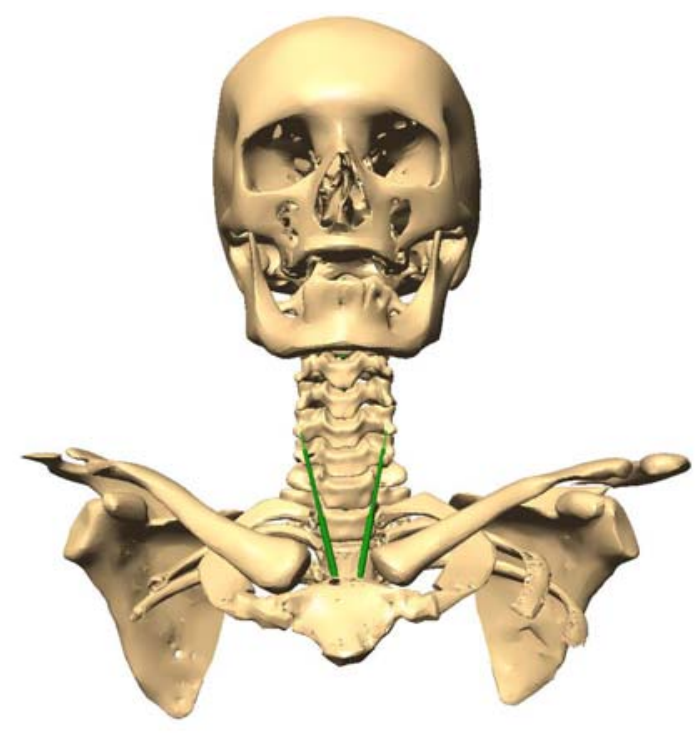

(B)

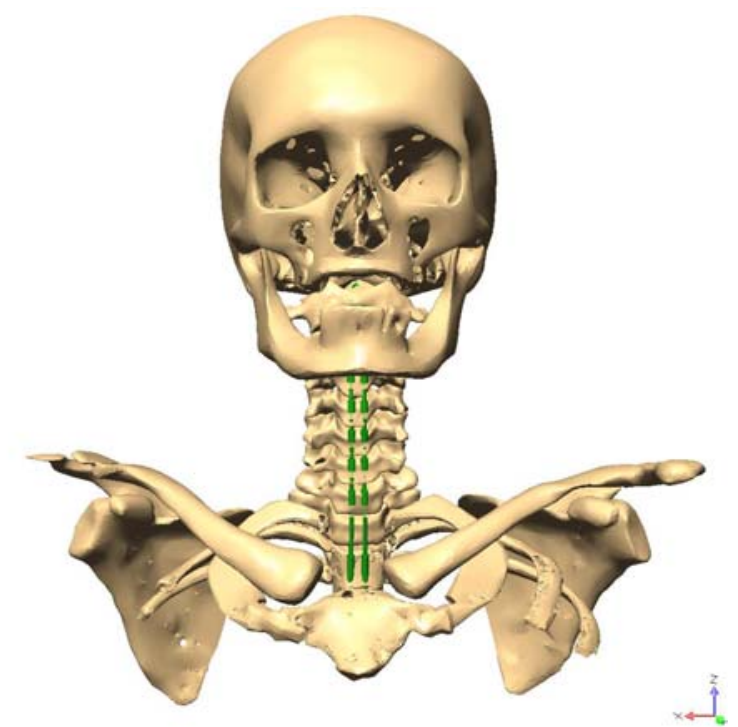

(D)

Figure A-1: Muscle longus capitis and colli. (A) Longus capitis. (B) Longus collis-inferior oblique. (C) Longus colli-superior oblique. (D) Longus colli-vertical. 


\section{Longus colli-vertical}

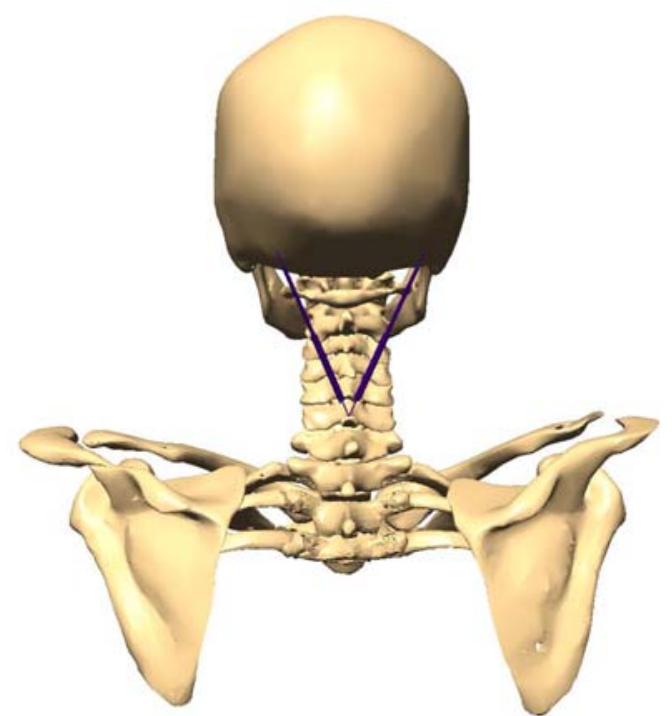

(A)

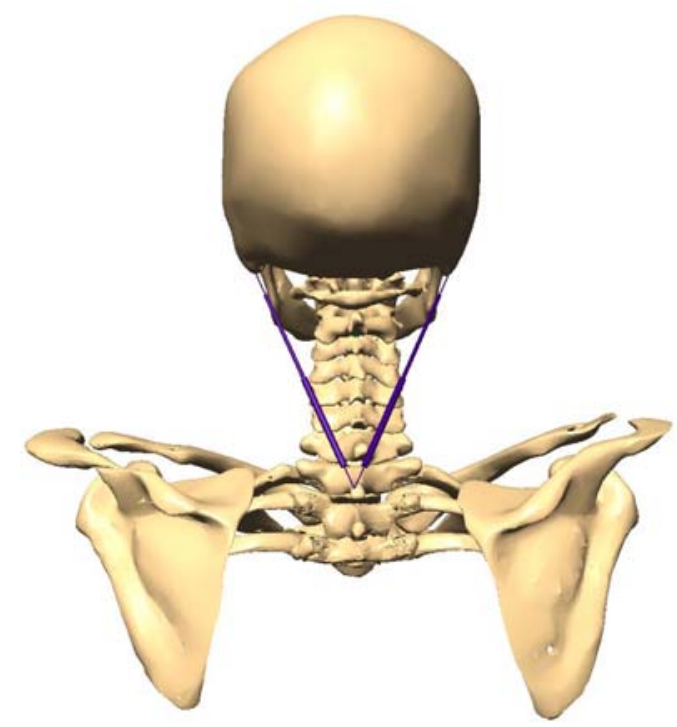

(B)

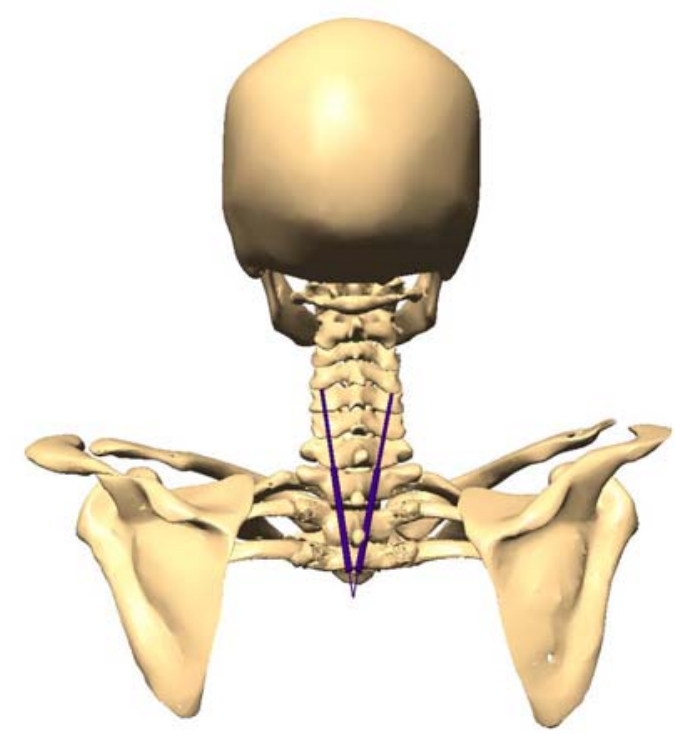

(C)

Figure A-2: Muscle longus colli-vertical. (A) Splenius capitis-medial. (B) Spl enius capitislateral. (C) Splenius cervicis. 


\section{Semispinalis}

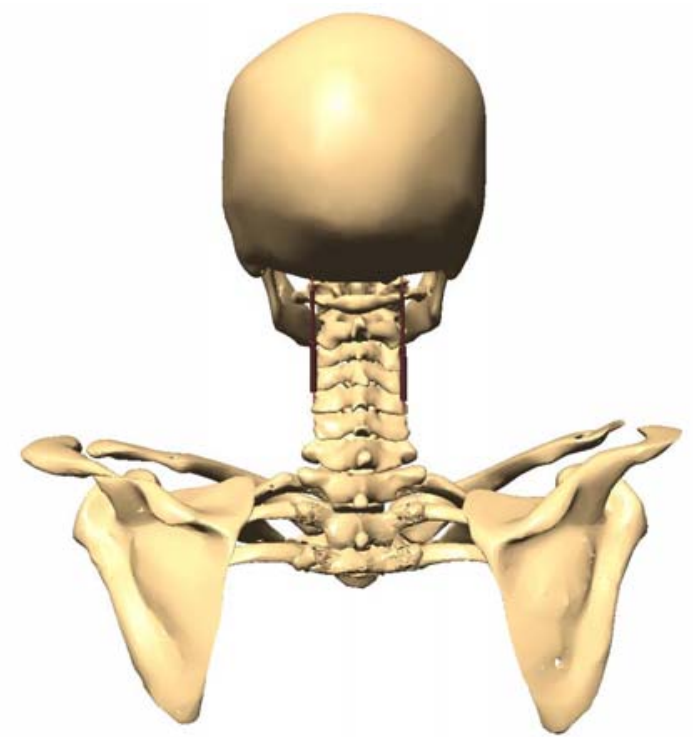

(A)

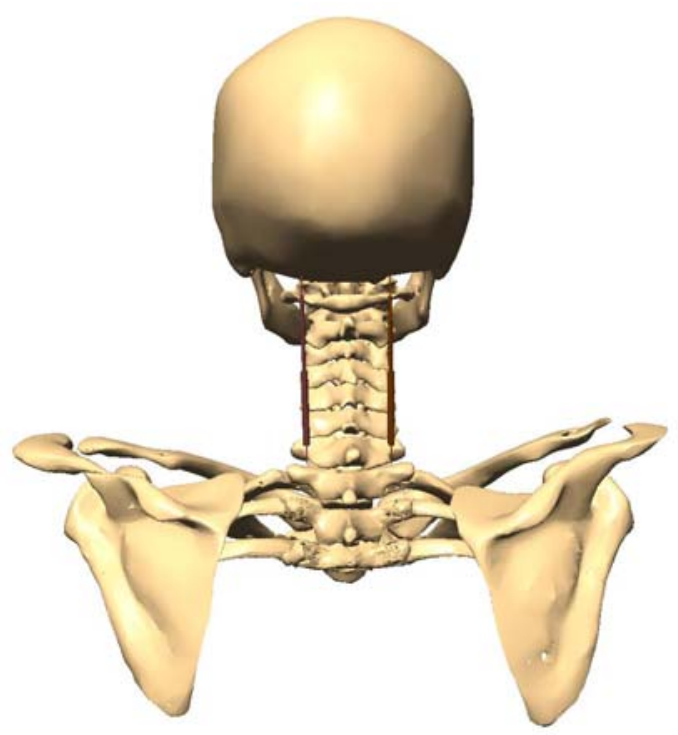

(B)

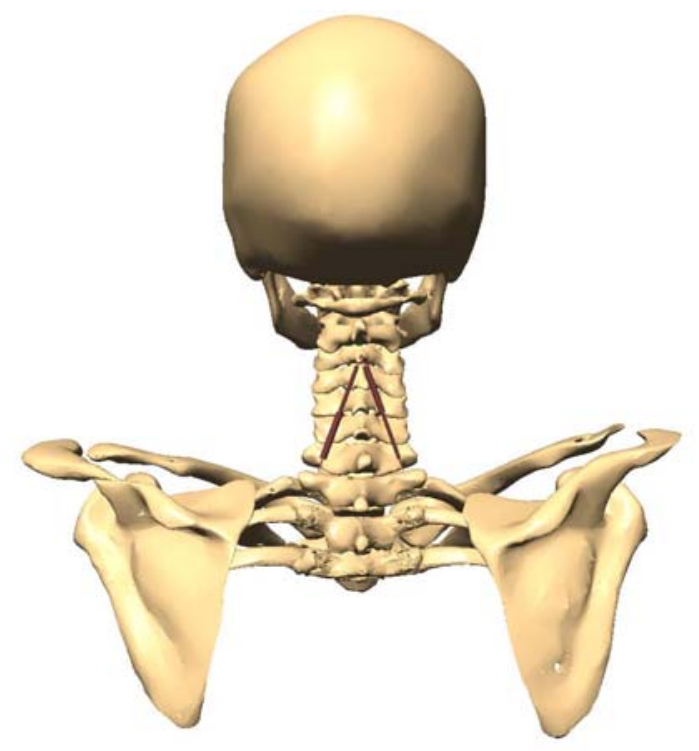

(C)

Figure A-3: Muscle semispinalis. (A) Semispinalis capitis-lateral. (B) Semispinalis capitismedial. (C) Semispinalis capitis cervicis. 


\section{Scalenes}

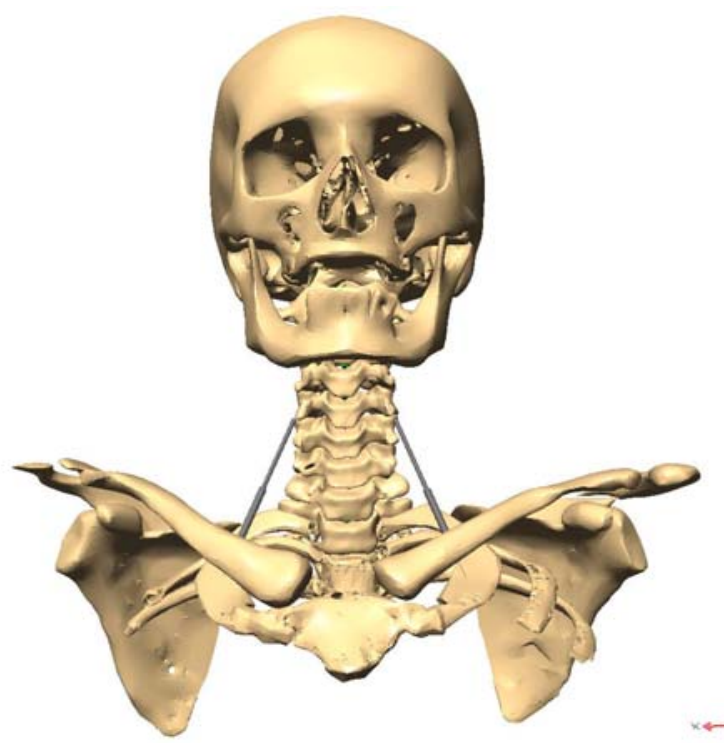

(A)

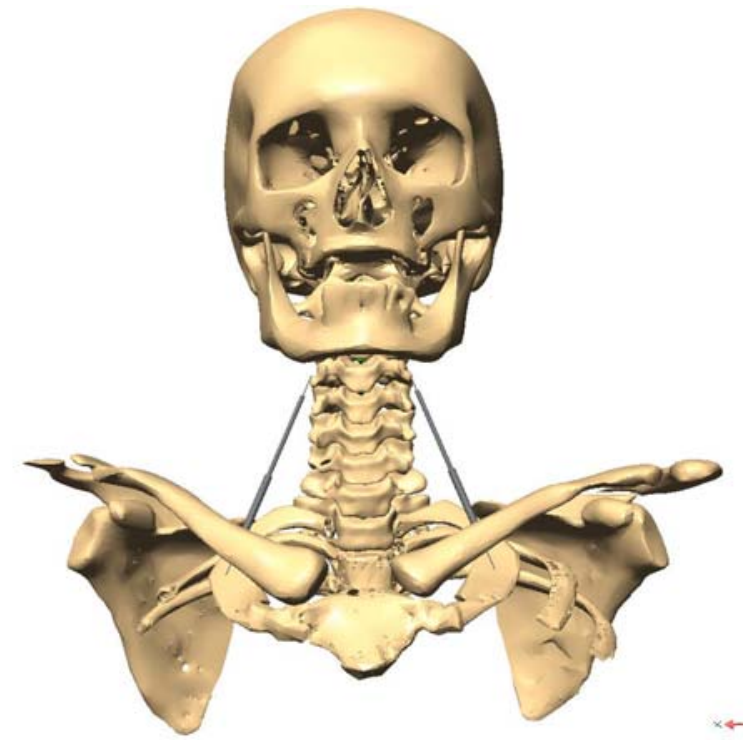

(B)

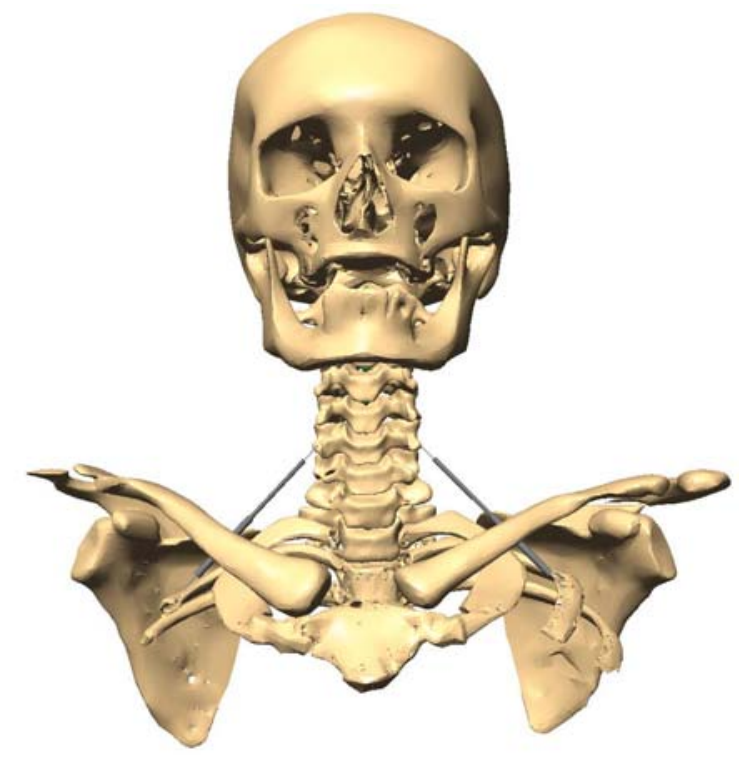

(C)

Figure A-4: Muscle scalenes. (A) Scalenes anterior. (B) Scalenes medius. (C) Scalenes posterior. 


\section{Erector spinae}

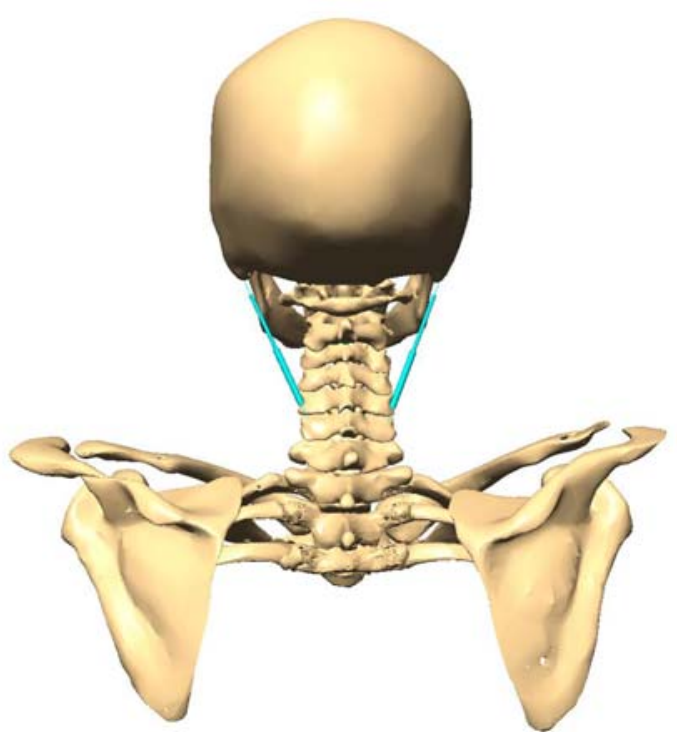

(A)

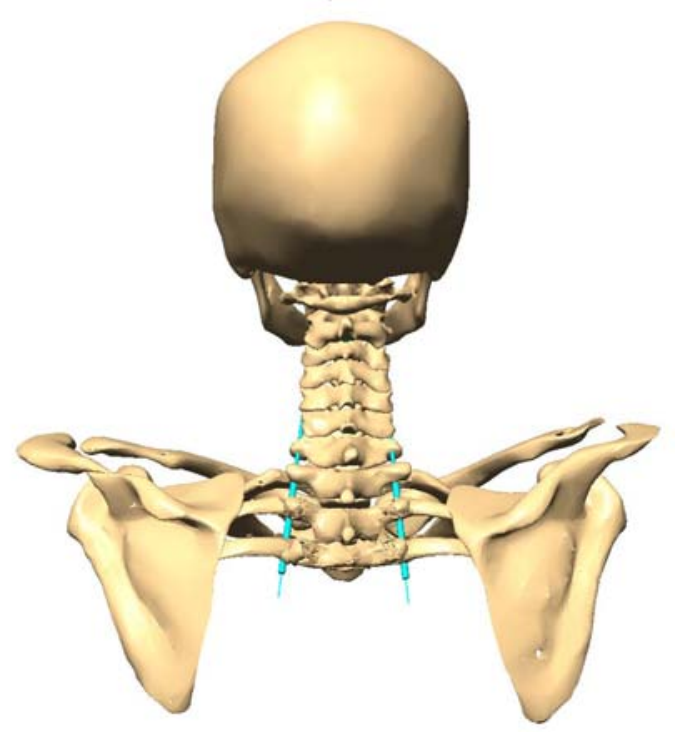

(B)

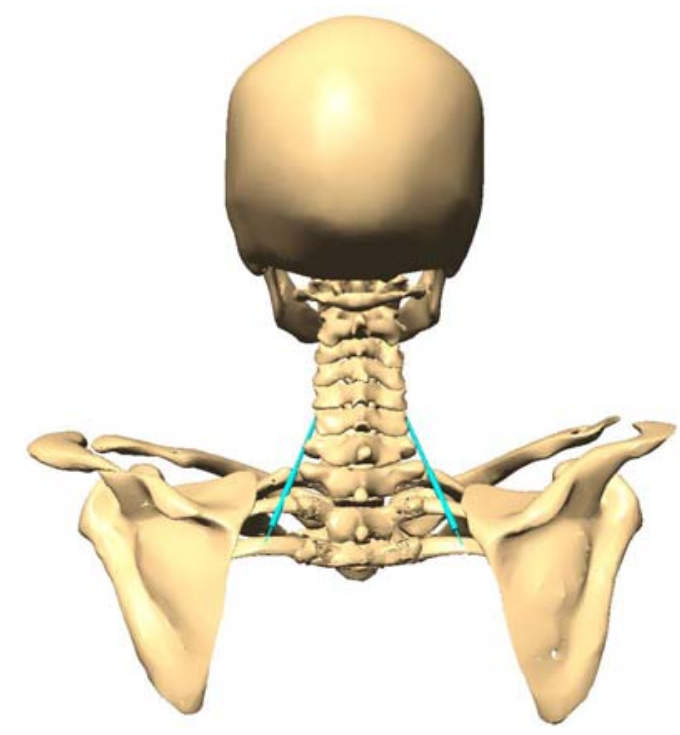

(C)

Figure A-5: Muscle erector spinae. (A) Longissimus capitis. (B) Longissmus cervicis. (C) Iliocostalis cervicis. 


\section{Sternocleidomastoid}

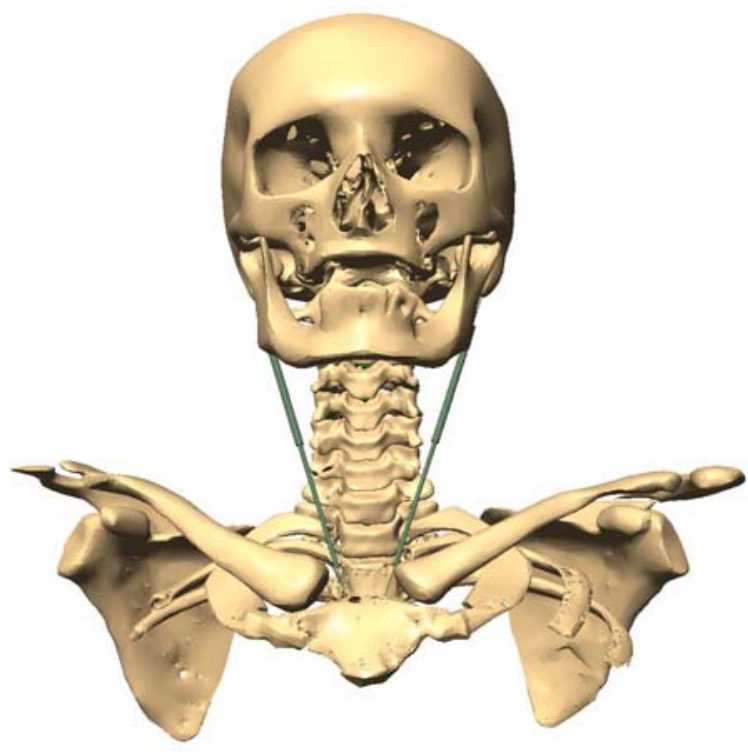

(A)

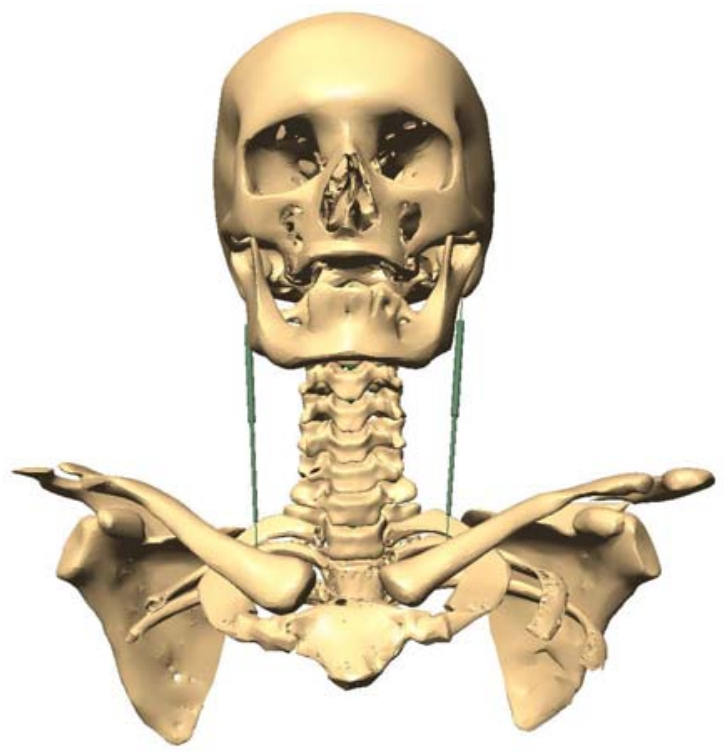

(B)

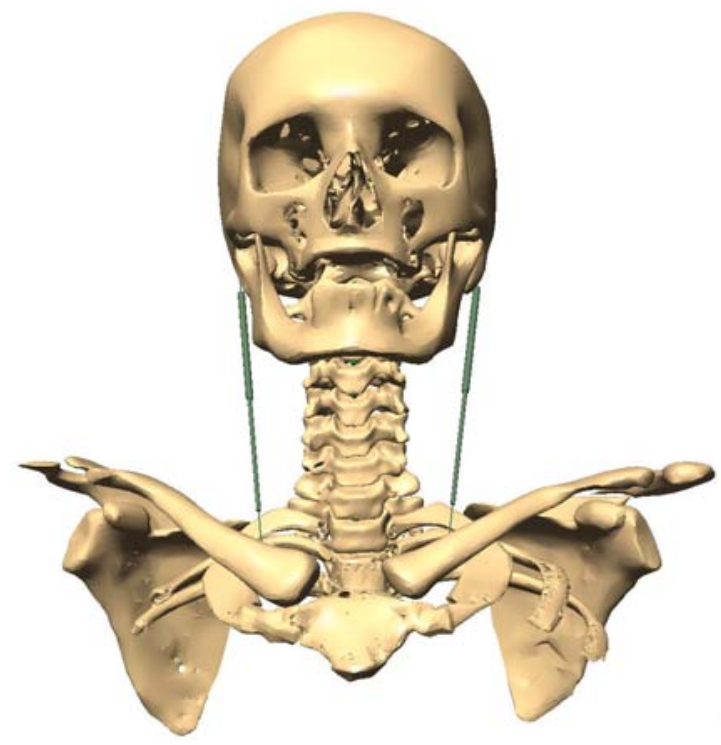

(C)

Figure A-6: Muscle sternocleidomastoid. (A) Sternomastoid. (B) Cleidomastoid. (C) Cleidooccipital. 


\section{Levator spinae}

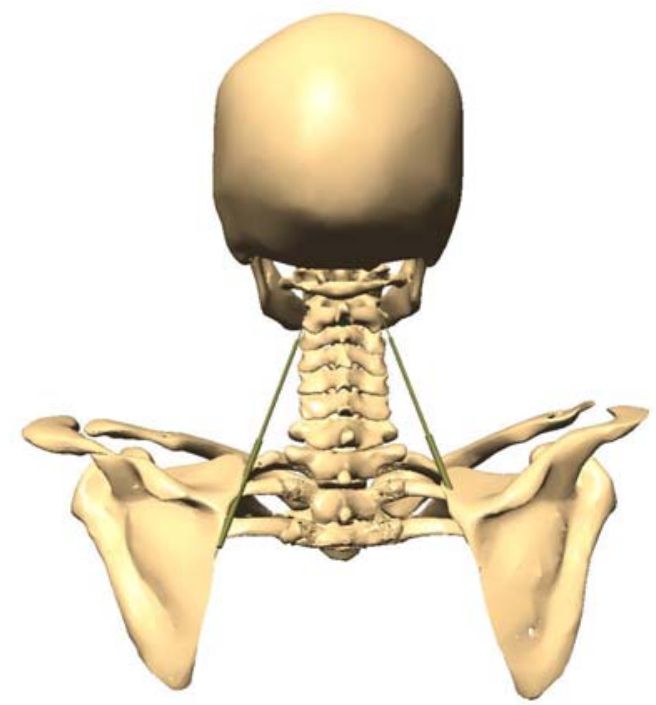

Figure A-7: Muscle levator spinae.

\section{Trapezius}

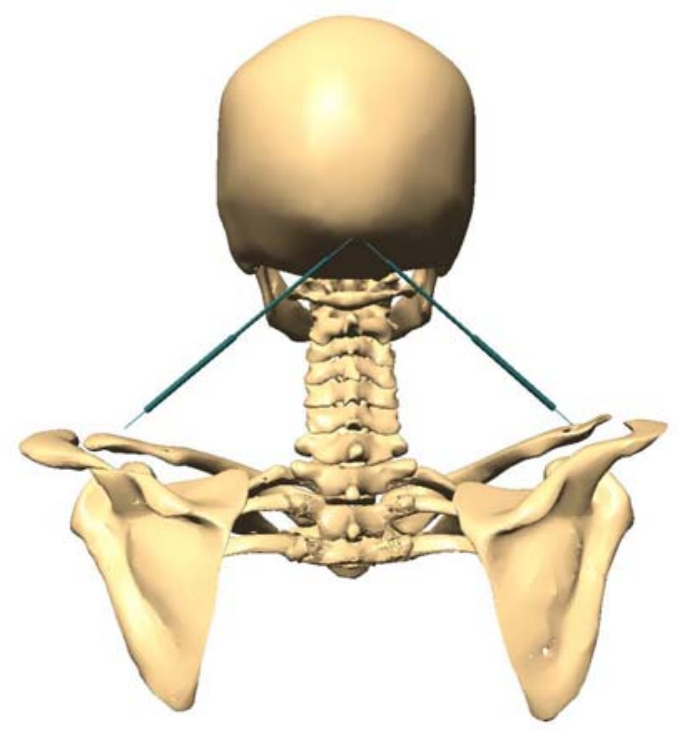

(A)

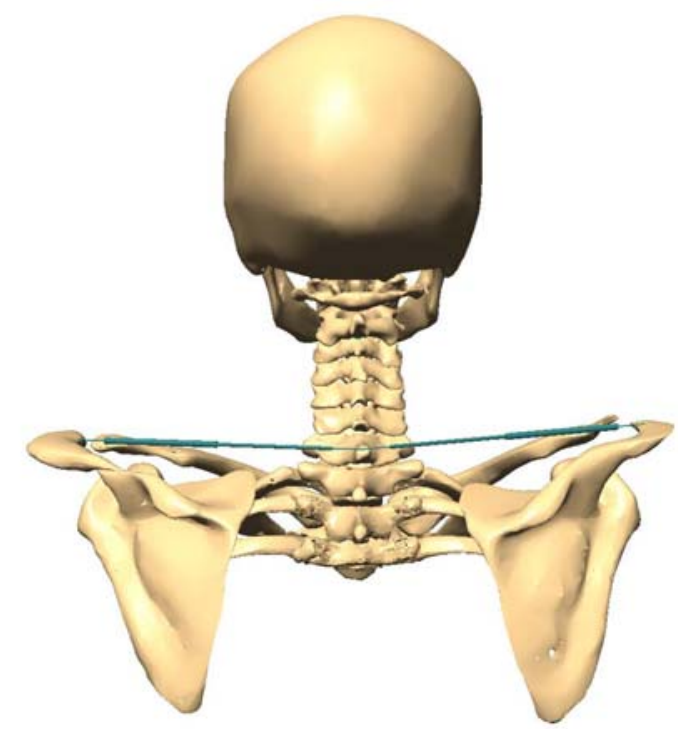

(B)

Figure A-8: Muscle trapezius. (A) Clavotrapezius. (B) Acromiotrapezius. 


\section{Suboccipital}

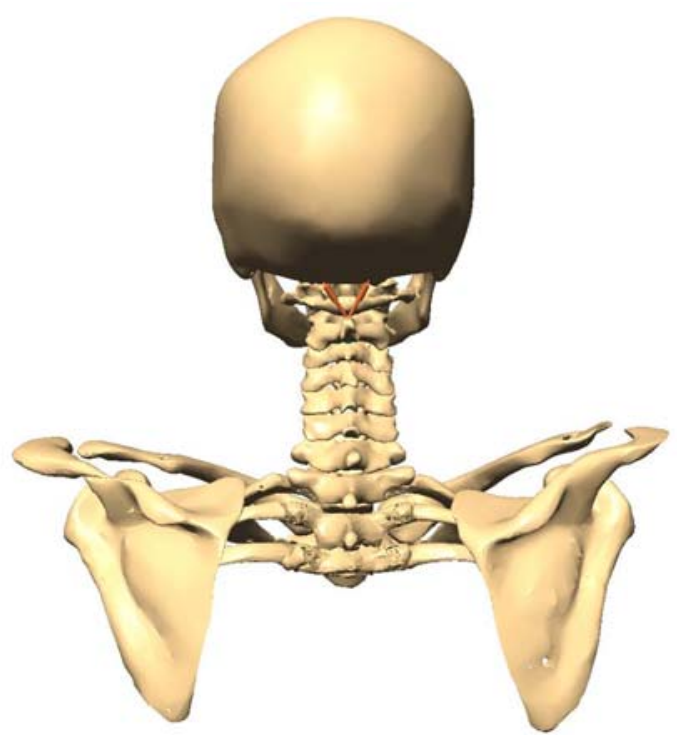

(A)

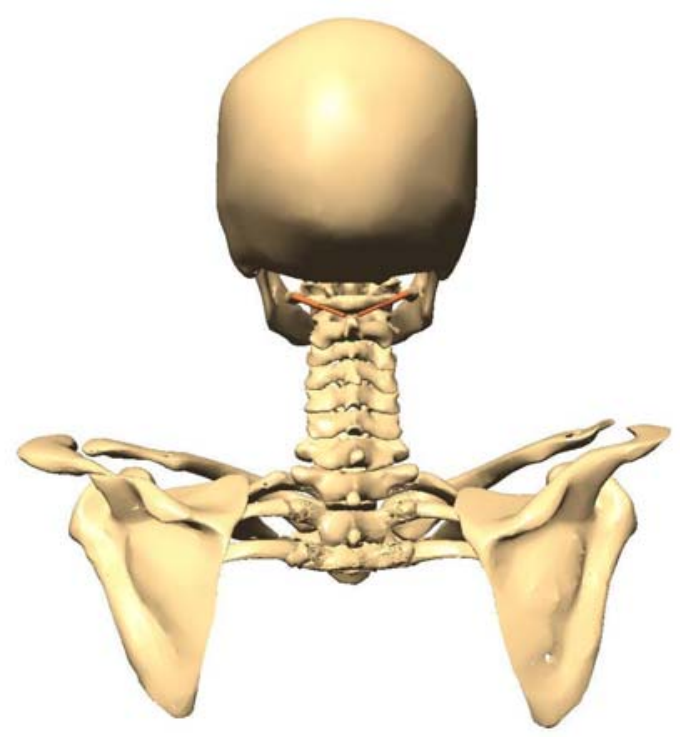

(C)

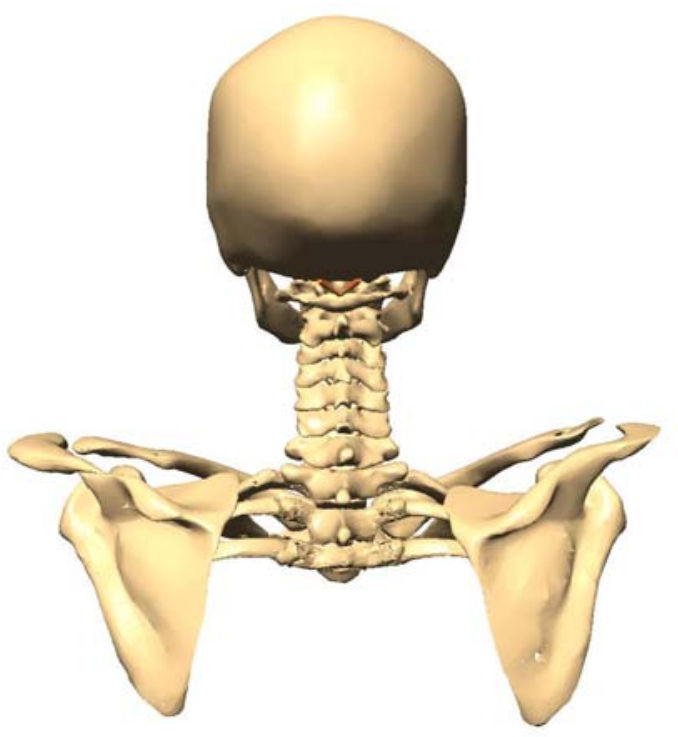

(B)

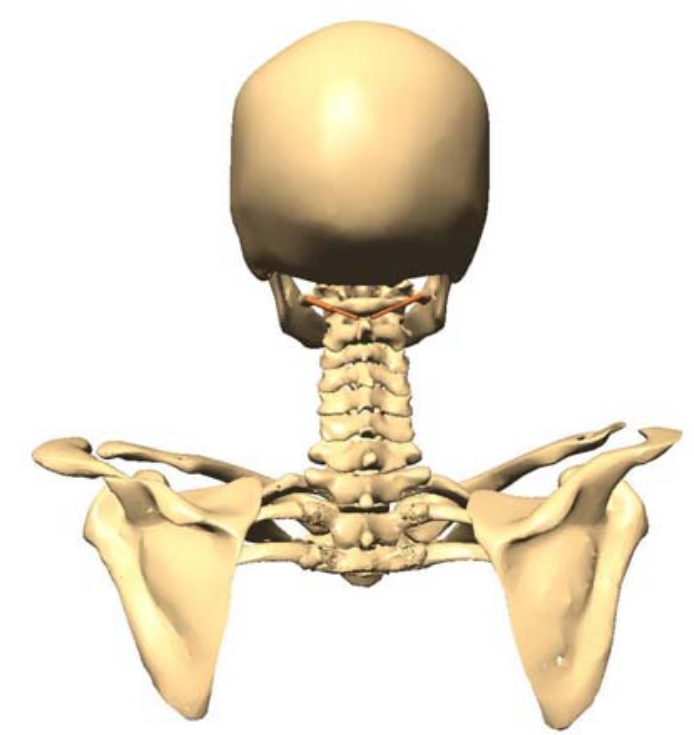

(D)

Figure A-9: Muscle suboccipital. (A) Rectus capitis posterior major. (B) Rectus capitis posterior minor. (C) Obliquus capitis superior. (D) Obliquus capitis inferior. 
Appendix B Motion segment unit rotations 


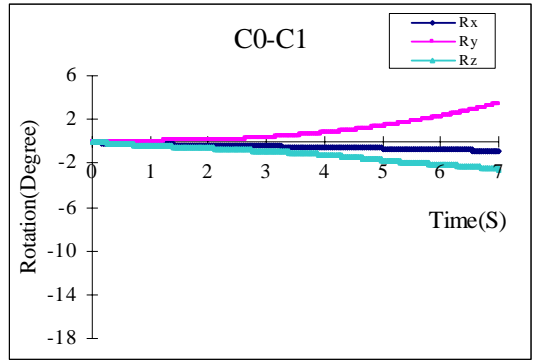

(A)

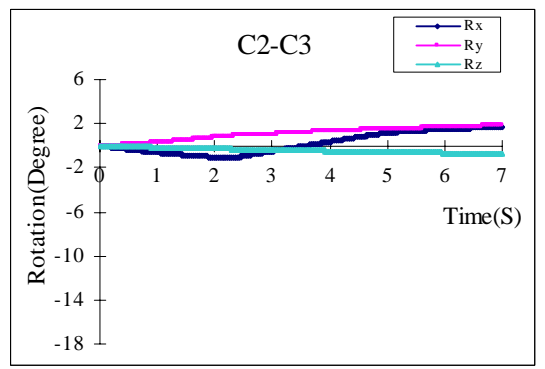

(C)

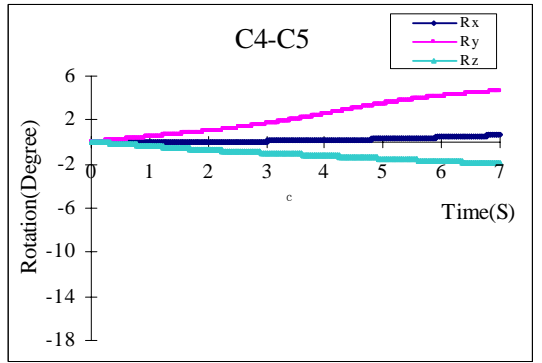

(E)

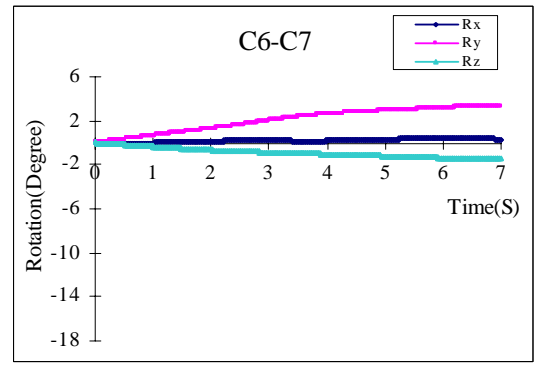

(G)

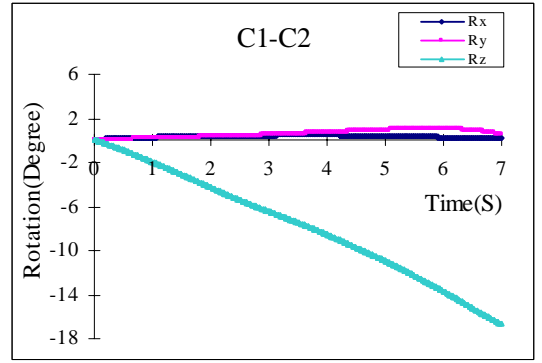

(B)

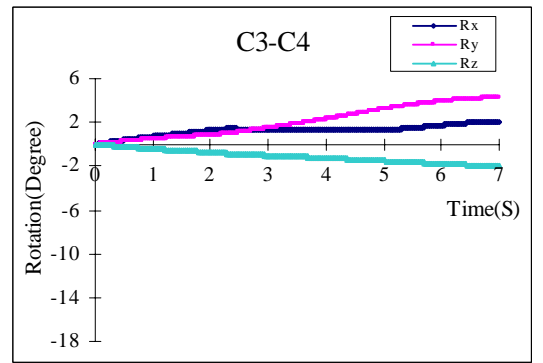

(D)

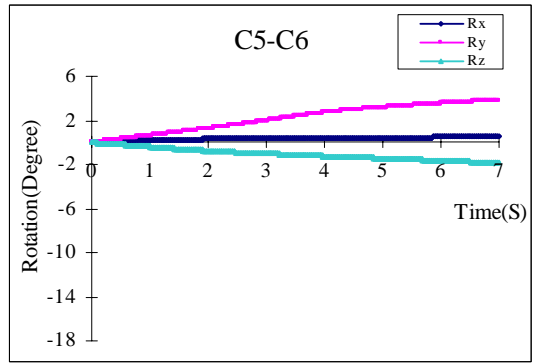

(F)

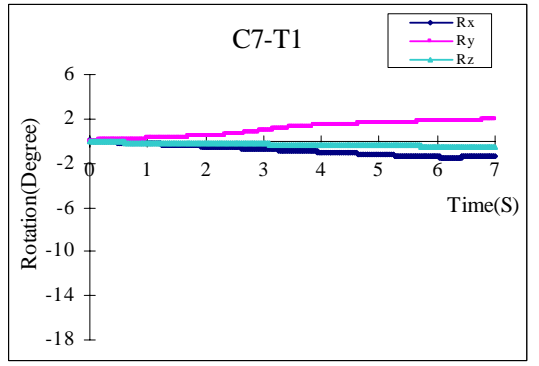

(H)

Figure B-1: The rotation versus time curves for each segment level under the "Concentric contraction muscles only” mode in axial rotation. (A) C0-C1. (B) C1-C2. (C) C2-C3. (D) C3C4. (E) C4-C5. (F). C5-C6. (G) C6-C7. (H) C7-T1. 


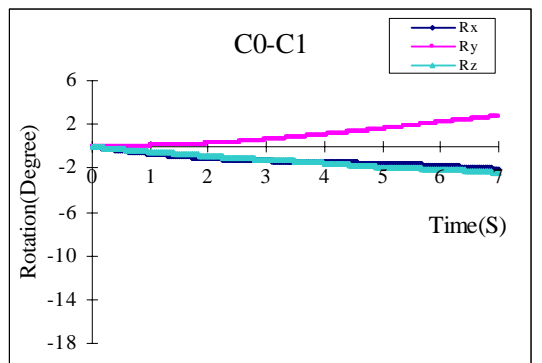

(A)

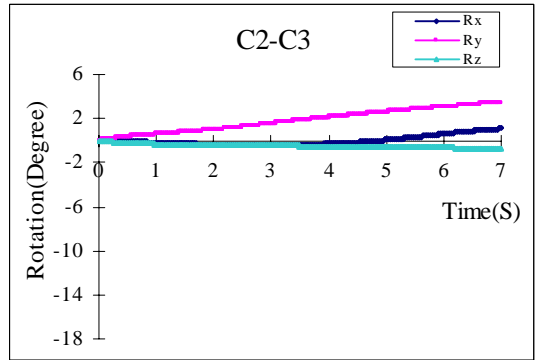

(C)

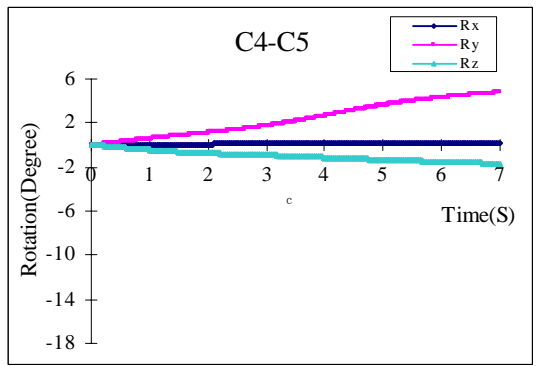

(E)

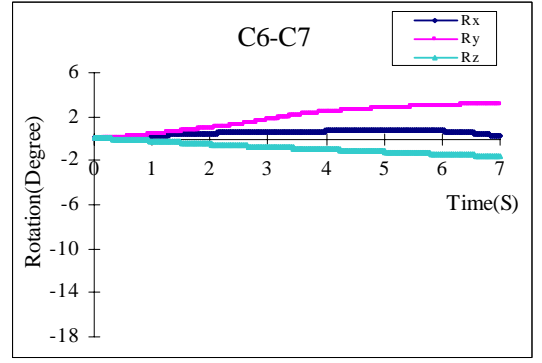

$(G)$

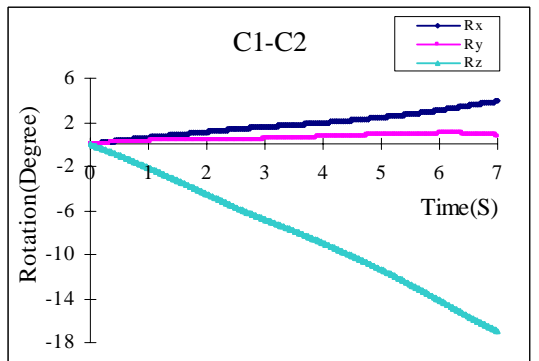

(B)

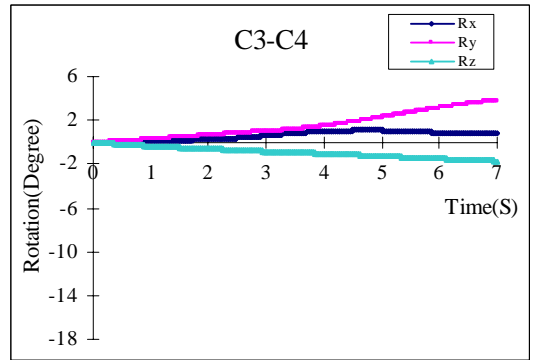

(D)

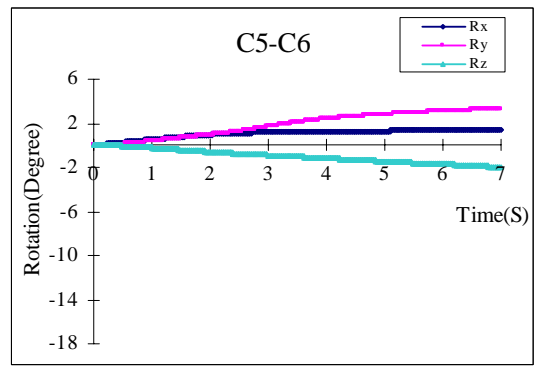

(F)

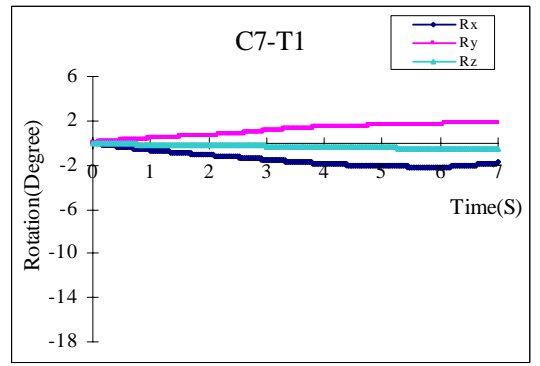

$(\mathrm{H})$

Figure B-2: The rotation versus time curves for each segment level under the "Elongation muscles only" mode in axial rotation. (A) C0-C1. (B) C1-C2. (C) C2-C3. (D) C3-C4. (E) C4C5. (F). C5-C6. (G) C6-C7. (H) C7-T1. 


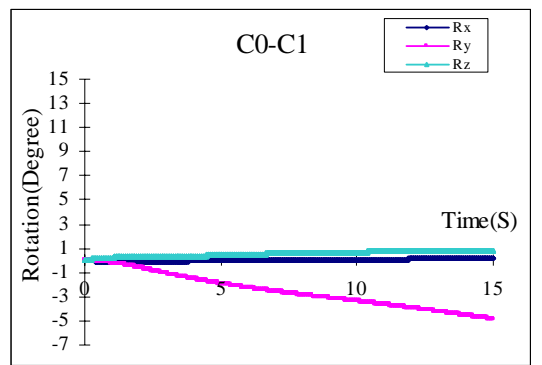

(A)

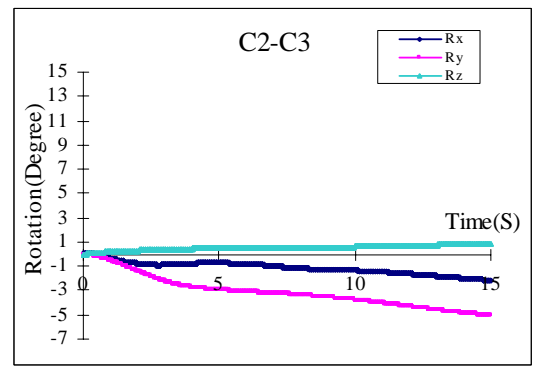

(C)

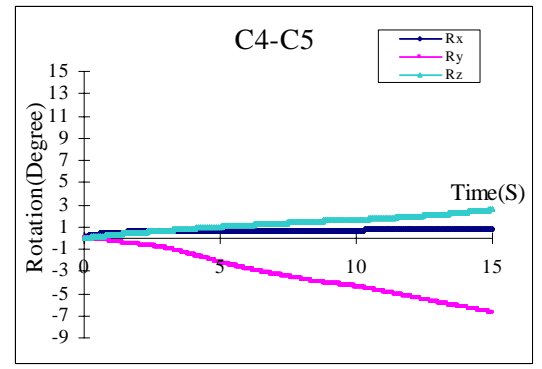

(E)

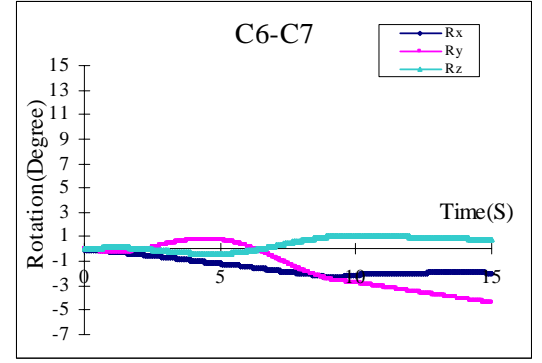

(G)

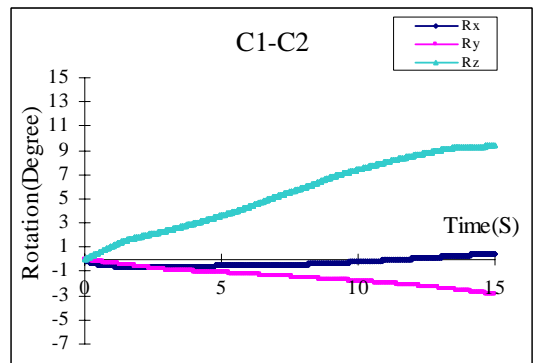

(B)

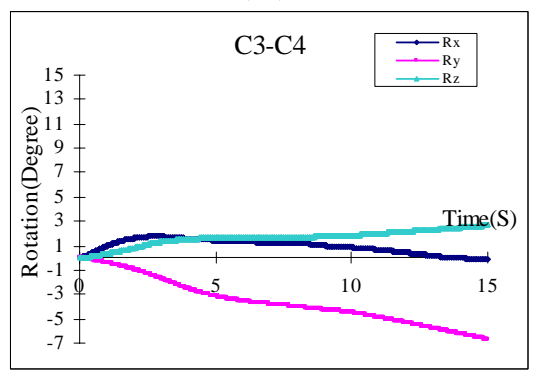

(D)

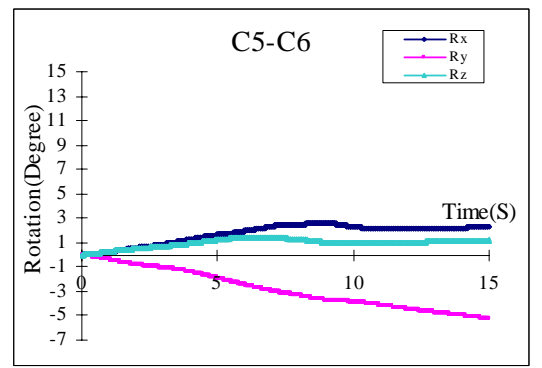

(F)

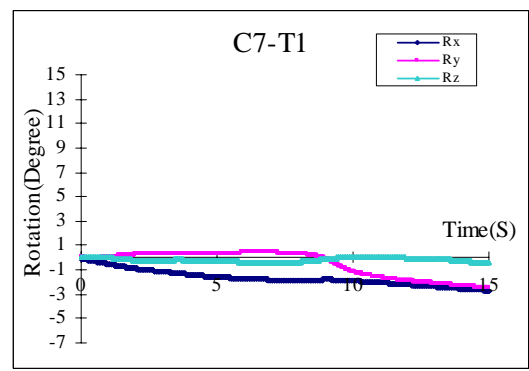

(H)

Figure B-3: The rotation versus time curves for each segment level under the "All muscles only” mode in lateral bending. (A) C0-C1. (B) C1-C2. (C) C2-C3. (D) C3-C4. (E) C4-C5. (F). C5-C6. (G) C6-C7. (H) C7-T1. 


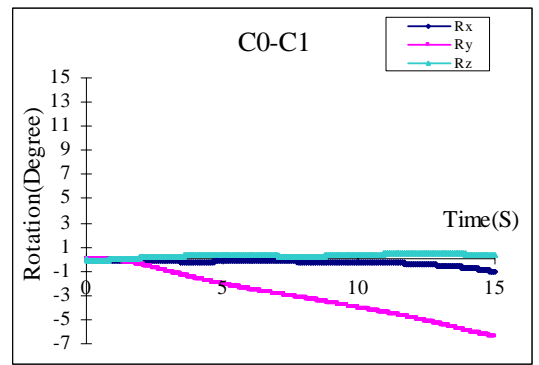

(A)

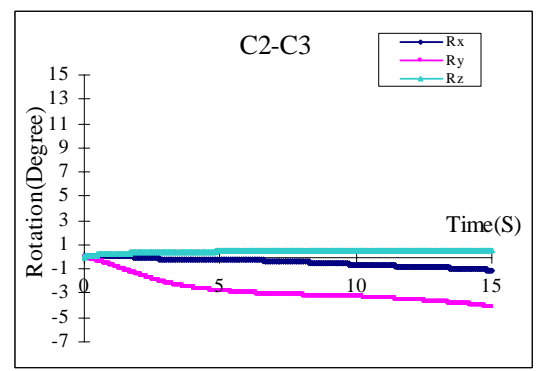

(C)

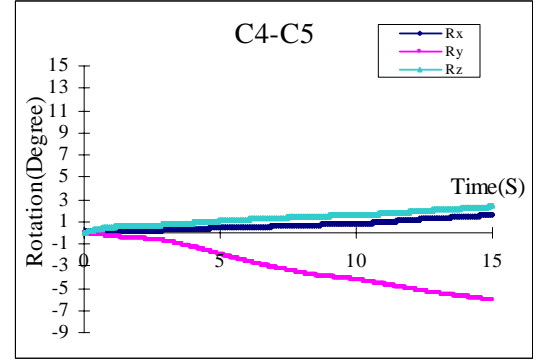

(E)

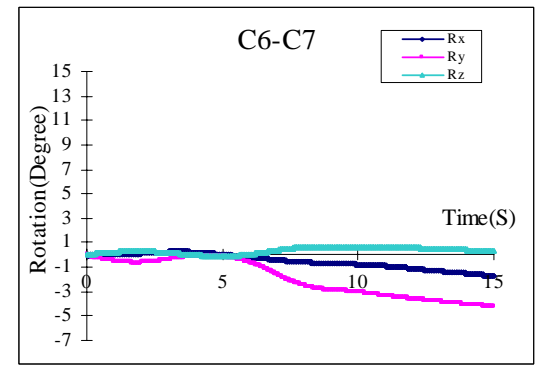

(G)

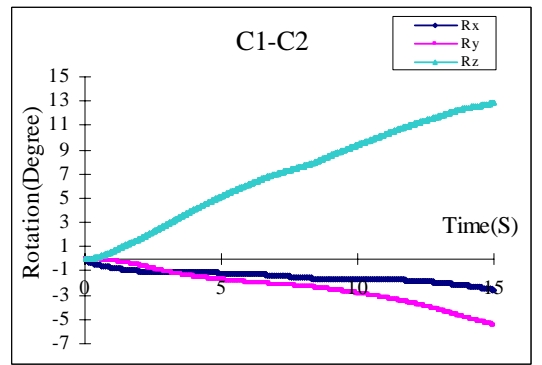

(B)

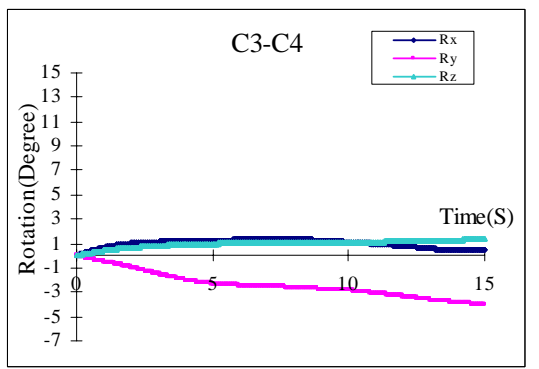

(D)

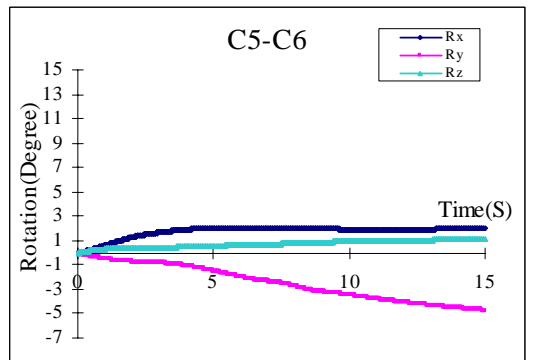

(F)

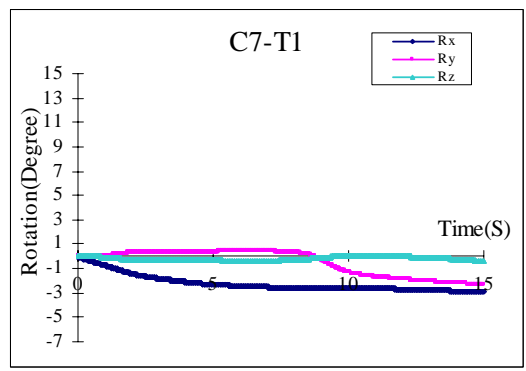

(H)

Figure B-4: The rotation versus time curves for each segment level under the "Concentric contraction muscles only" mode in lateral bending. (A) C0-C1. (B) C1-C2. (C) C2-C3. (D) C3-C4. (E) C4-C5. (F). C5-C6. (G) C6-C7. (H) C7-T1. 


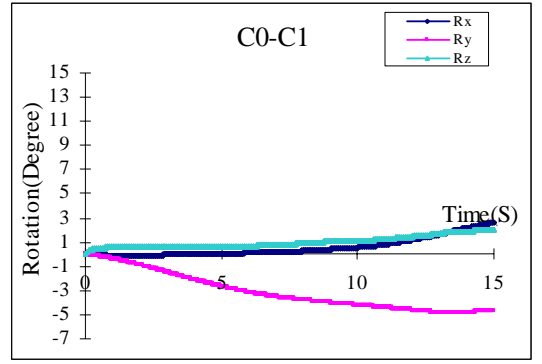

(A)

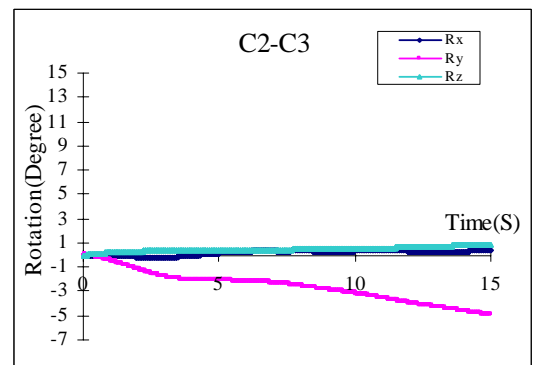

(C)

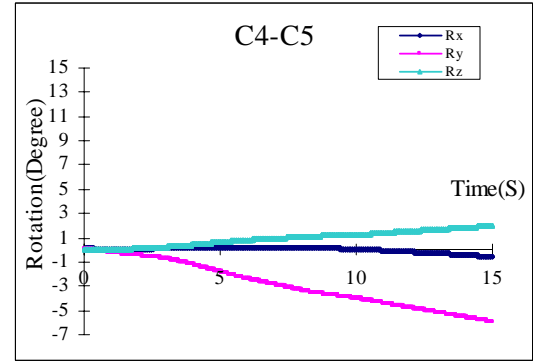

(E)

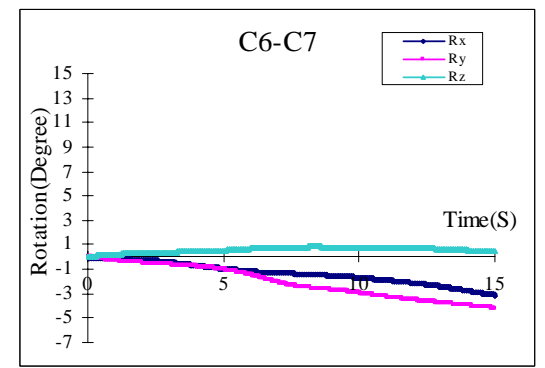

$(G)$

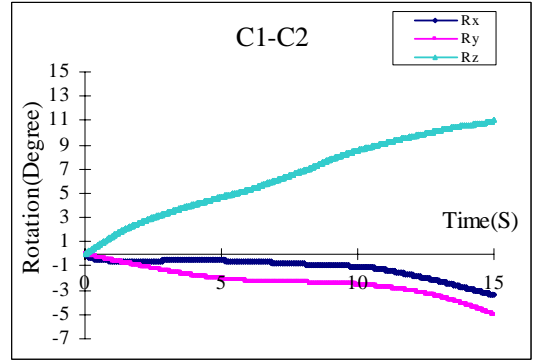

(B)

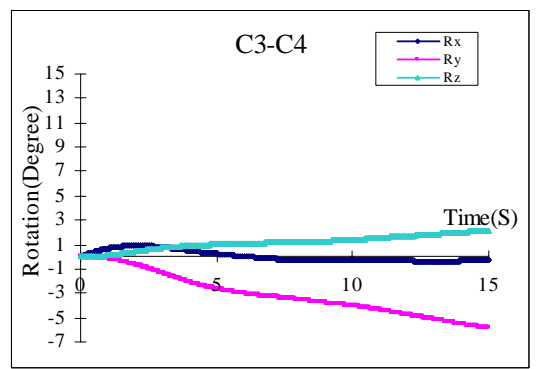

(D)

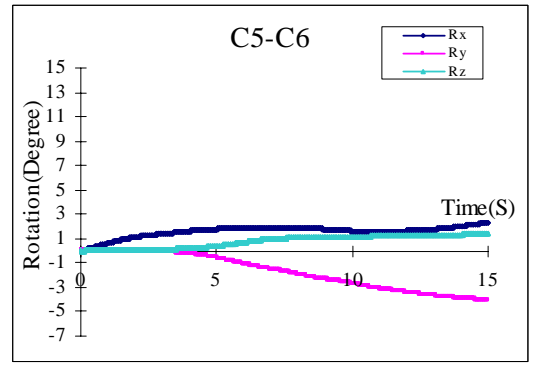

(F)

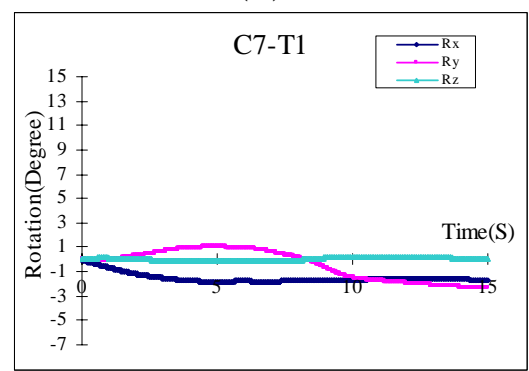

(H)

Figure B-5: The rotation versus time curves for each segment level under the "Elongation muscles only" mode in lateral bending. (A) C0-C1. (B) C1-C2. (C) C2-C3. (D) C3-C4. (E) C4-C5. (F). C5-C6. (G) C6-C7. (H) C7-T1. 


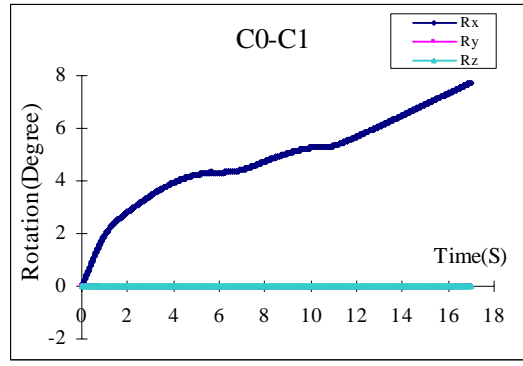

(A)

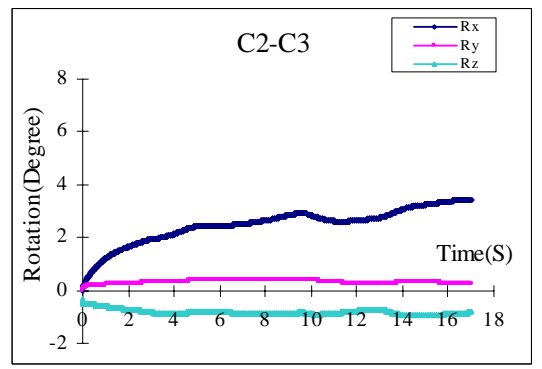

(C)

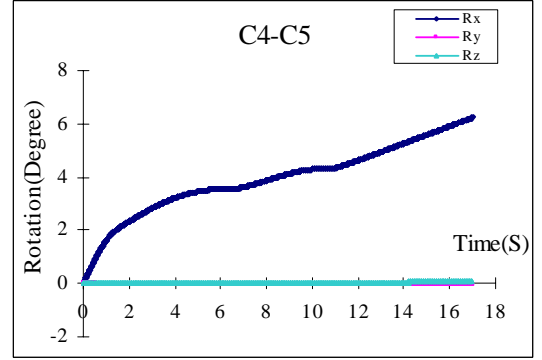

(E)

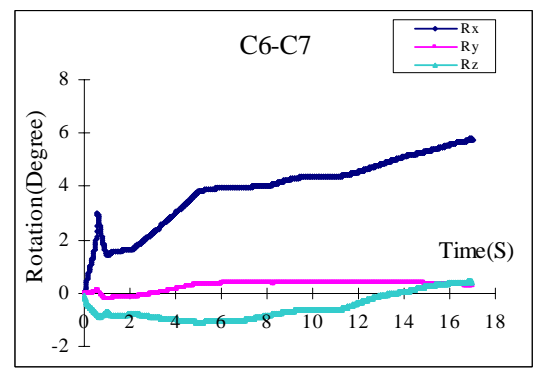

$(\mathrm{G})$

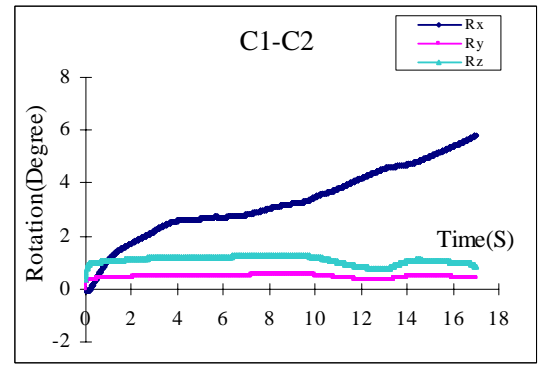

(B)

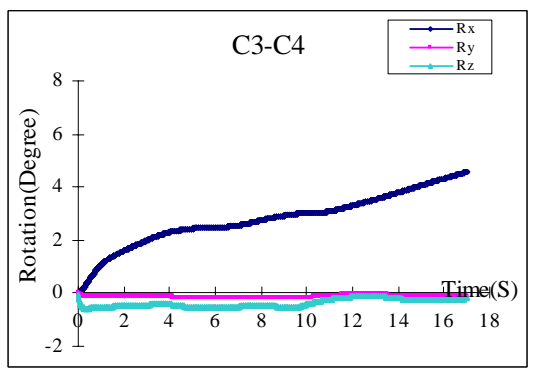

(D)

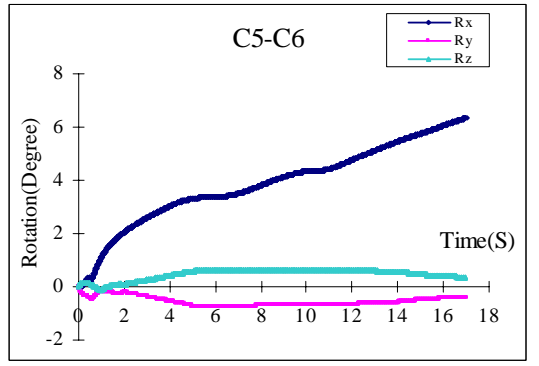

(F)

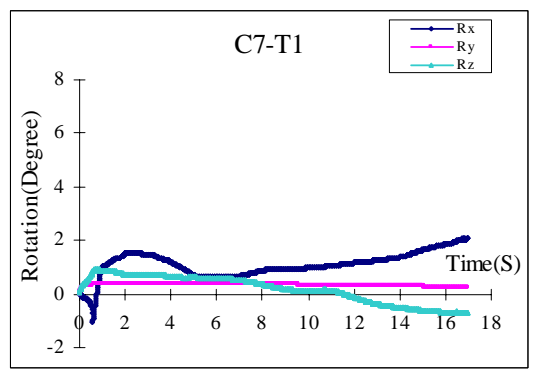

(H)

Figure B-6: The rotation versus time curves for each segment level under the "All muscles only" mode in extension. (A) C0-C1. (B) C1-C2. (C) C2-C3. (D) C3-C4. (E) C4-C5. (F). C5C6. (G) C6-C7. (H) C7-T1. 


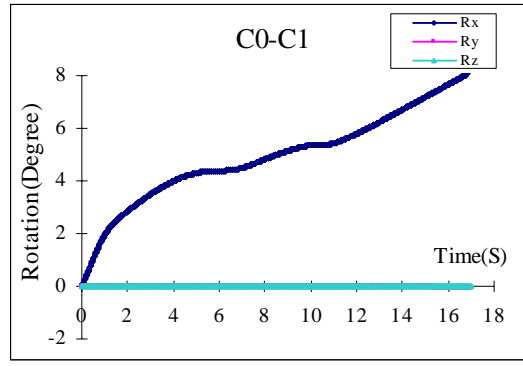

(A)

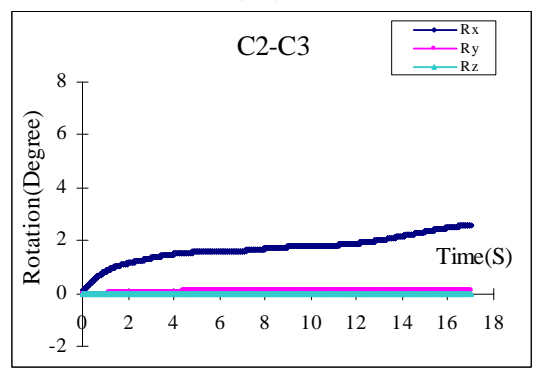

(C)

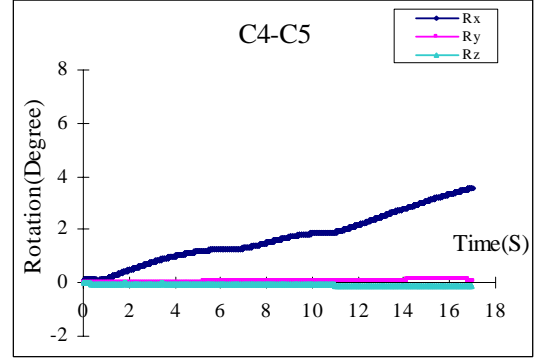

(E)

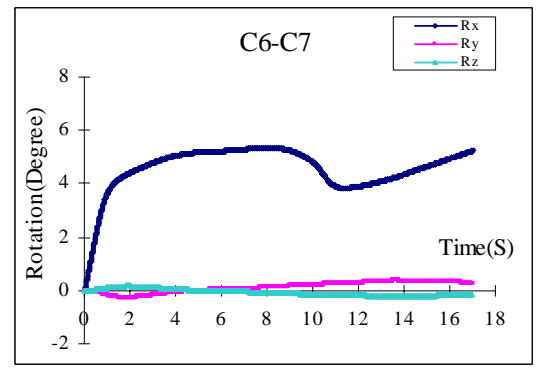

$(\mathrm{G})$

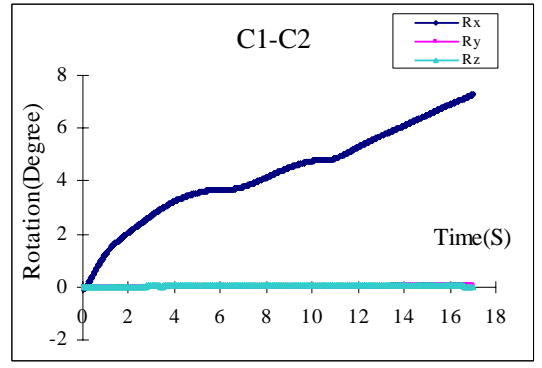

(B)

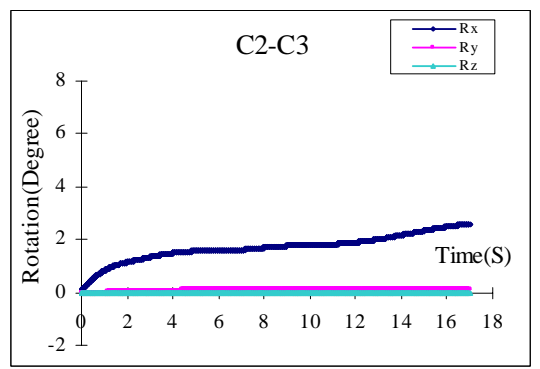

(D)

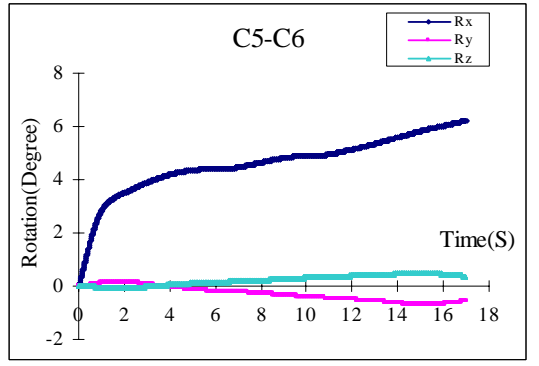

(F)

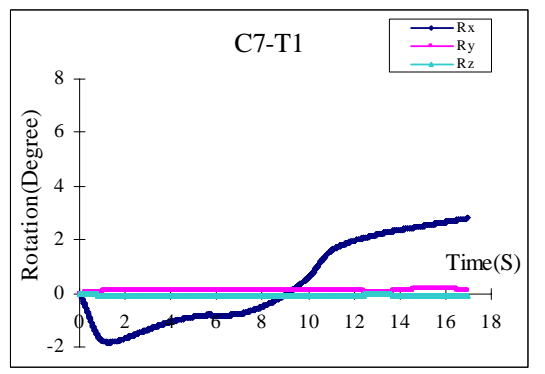

(H)

Figure B-7: The rotation versus time curves for each segment level under the "Concentric contraction muscles only" mode in extension. (A) C0-C1. (B) C1-C2. (C) C2-C3. (D) C3-C4. (E) C4-C5. (F). C5-C6. (G) C6-C7. (H) C7-T1. 


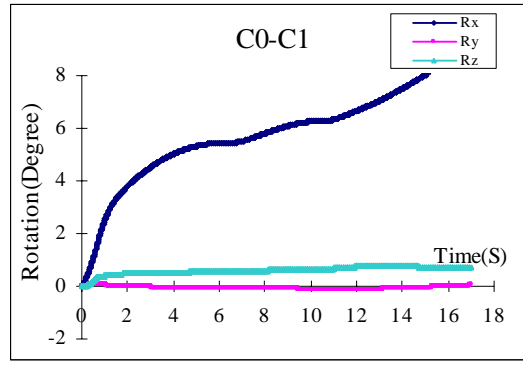

(A)

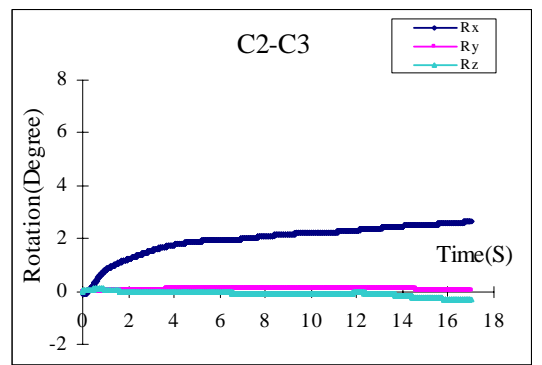

(C)

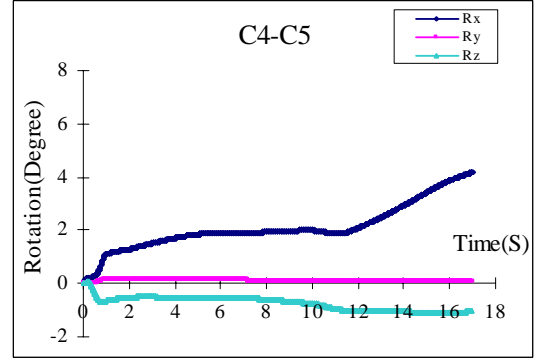

(E)

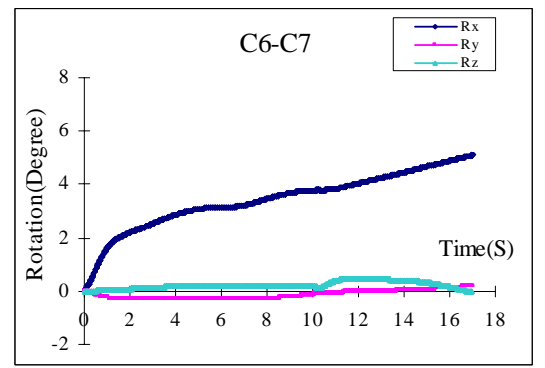

$(\mathrm{G})$

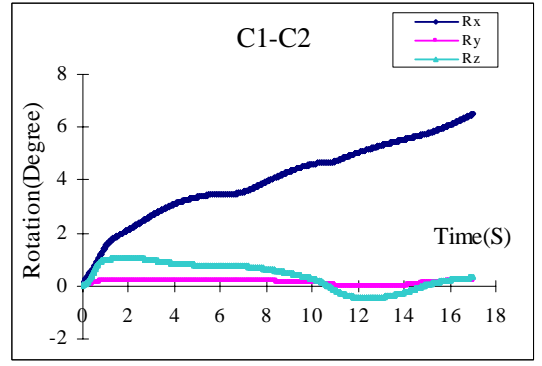

(B)

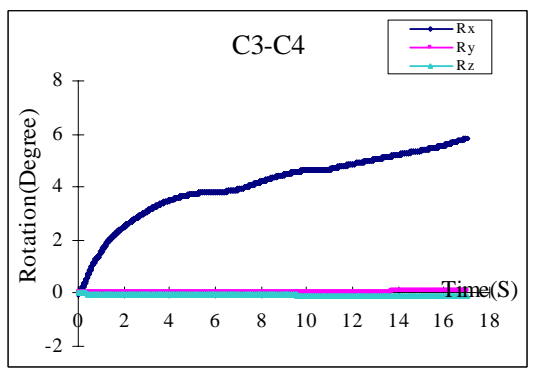

(D)

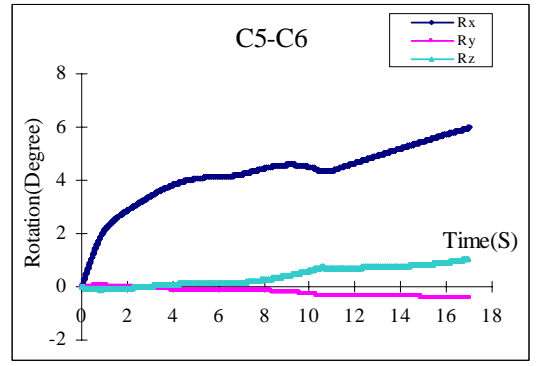

(F)

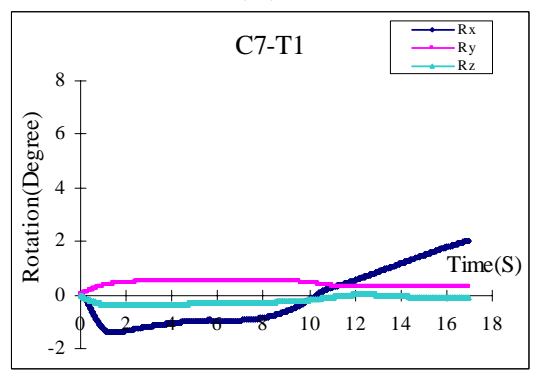

(H)

Figure B-8: The rotation versus time curves for each segment level under the "Elongation muscles only" mode in extension. (A) C0-C1. (B) C1-C2. (C) C2-C3. (D) C3-C4. (E) C4-C5. (F). C5-C6. (G) C6-C7. (H) C7-T1. 


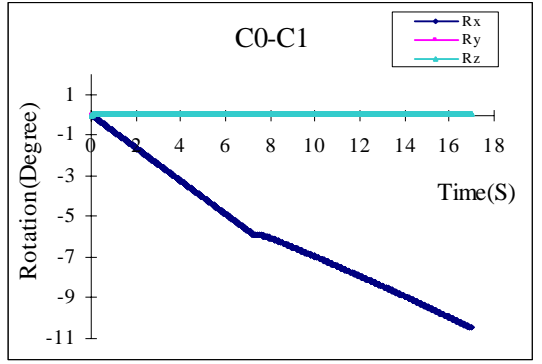

(A)

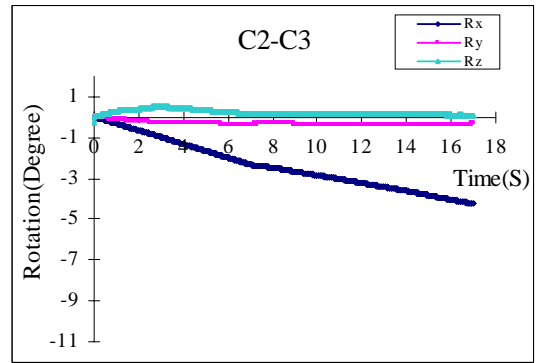

(C)

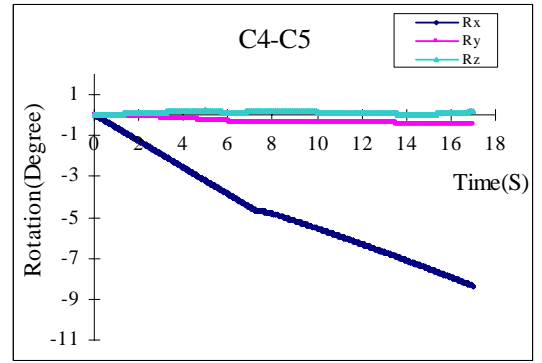

(E)

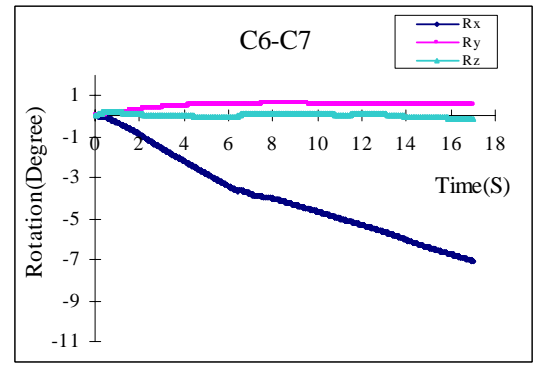

$(\mathrm{G})$

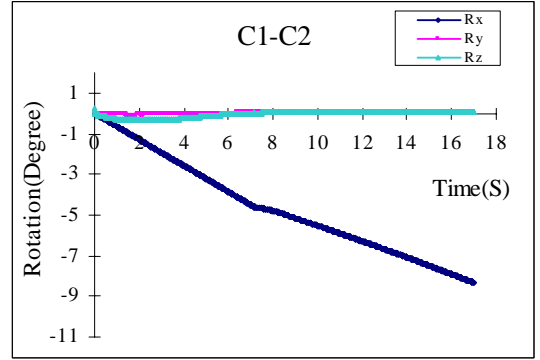

(B)

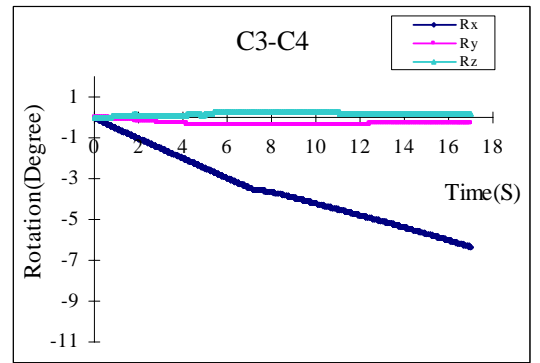

(D)

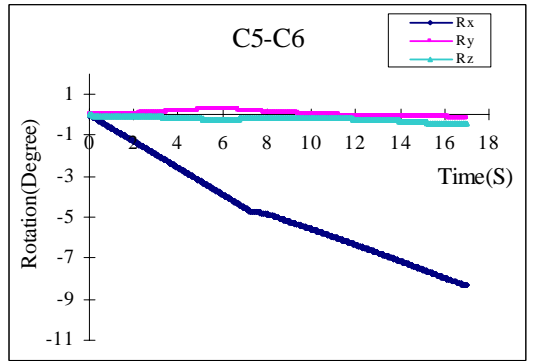

(F)

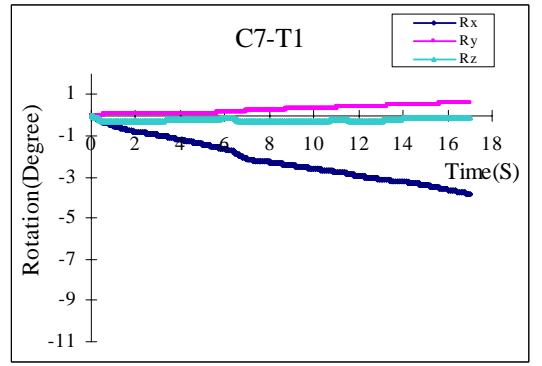

(H)

Figure B-9: The rotation versus time curves for each segment level under the "All muscles only" mode in flexion. (A) C0-C1. (B) C1-C2. (C) C2-C3. (D) C3-C4. (E) C4-C5. (F). C5-C6. (G) C6-C7. (H) C7-T1. 


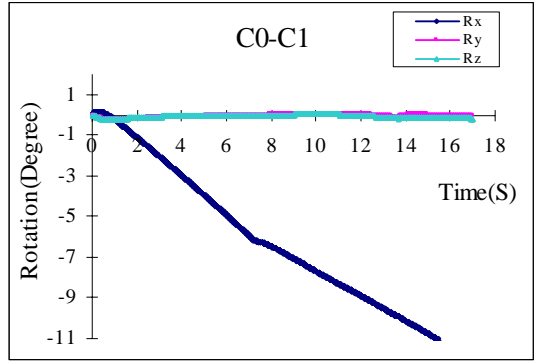

(A)

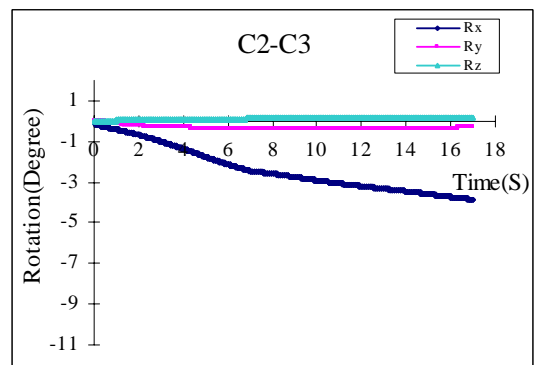

(C)

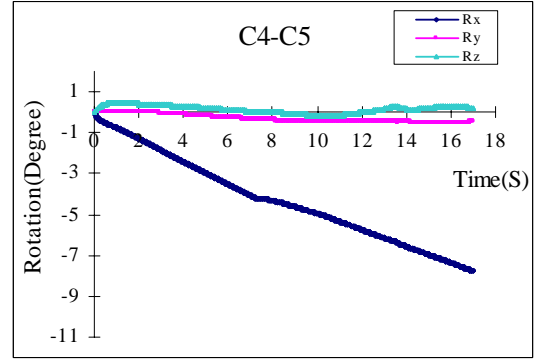

(E)

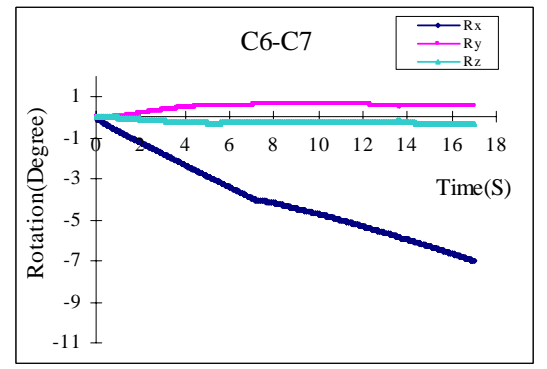

$(\mathrm{G})$

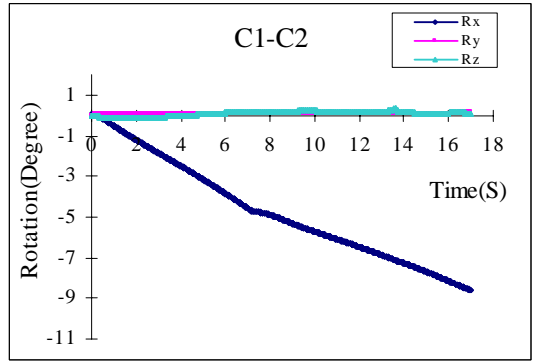

(B)

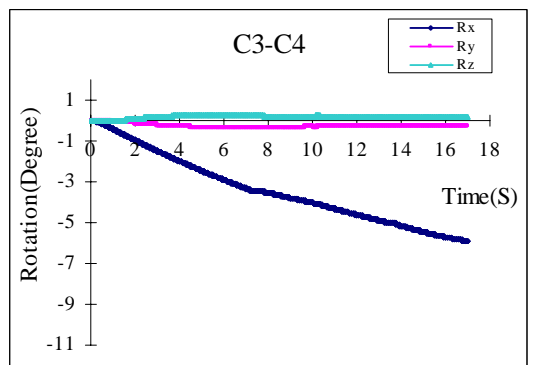

(D)

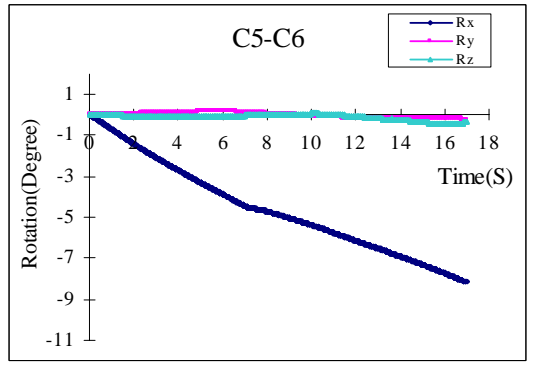

(F)

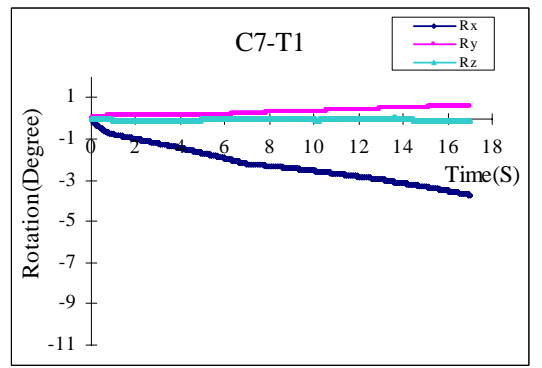

(H)

Figure B-10: The rotation versus time curves for each segment level under the "Concentric contraction muscles only" mode in flexion. (A) C0-C1. (B) C1-C2. (C) C2-C3. (D) C3-C4. (E) C4-C5. (F). C5-C6. (G) C6-C7. (H) C7-T1. 


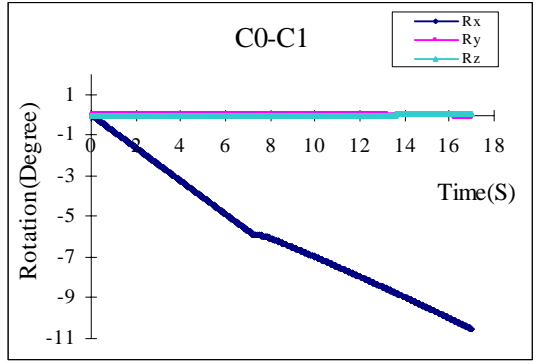

(A)

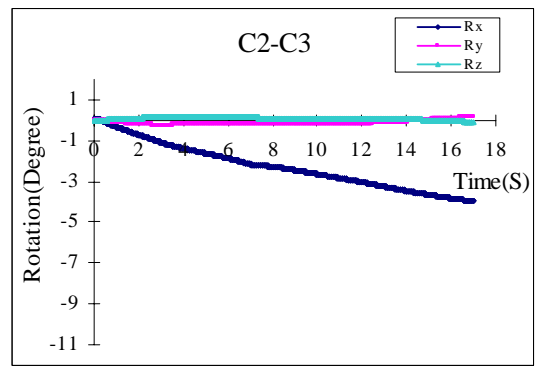

(C)

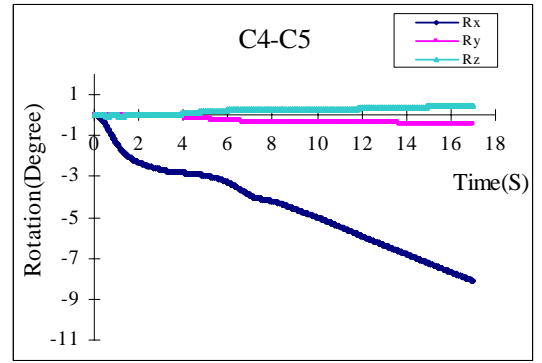

(E)

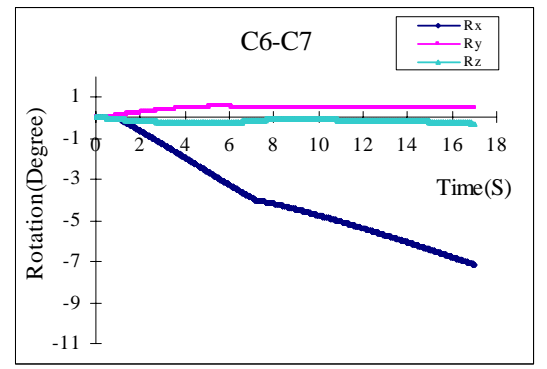

$(G)$

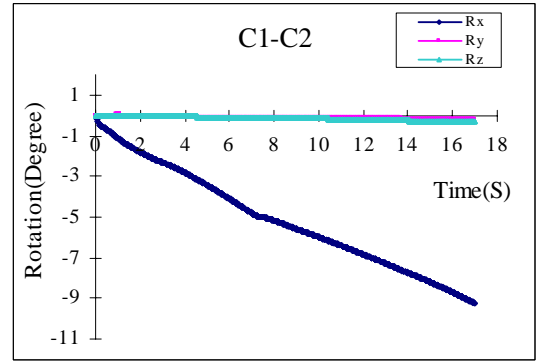

(B)

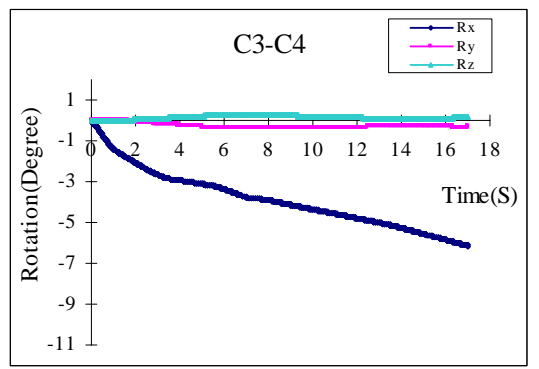

(D)

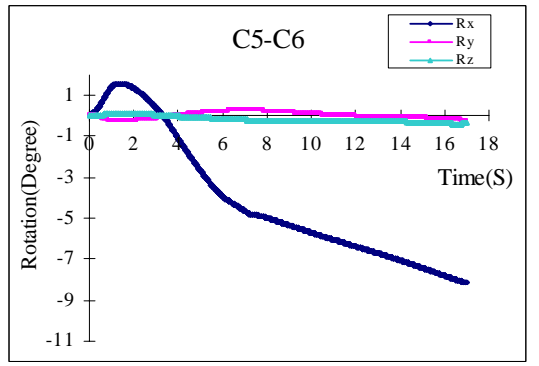

(F)

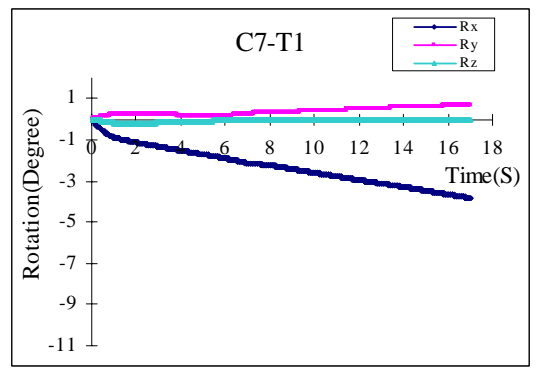

(H)

Figure B-11: The rotation versus time curves for each segment level under the "Elongation muscles only" mode in extension. (A) C0-C1. (B) C1-C2. (C) C2-C3. (D) C3-C4. (E) C4-C5. (F). C5-C6. (G) C6-C7. (H) C7-T1. 


\section{Vita}

Jun Yan was born in Guangzhou, the capital of Guangdong province, P.R China on Aug 5, 1977. After graduating from Zhixin High School, she entered Southeast University, Nanjing, P.R. China in 1996, and earned a Bachelor of Science Degree in Mechanical Engineering in 2000. She worked as a research assistant in Southeast University, Nanjing, China, and South China University of Technology, Guangzhou, China from 2000-2001 and 2001-2003, respectively. In the Fall of 2003, she came to the United States and was first enrolled in the Department of Mechanical Engineering at the University of Memphis. She then joined the Joint Biomedical Engineering Program at the University of Tennessee Health Science Center and the University of Memphis in August of 2004. At that time, she joined the Joint-Implant Biomechanics Laboratory under the supervision of Denis J. DiAngelo Ph.D. to continue her Master's Degree. After graduation, Jun plans to continue her Ph.D in Biomedical Engineering or work as a bioengineer in industry. 